Supporting Information for

\title{
Multinuclear Clusters of Manganese and Lithium with Silsesquioxane-derived Ligands: Synthesis and Ligand Rearrangement by Dioxygen-and Base-mediated Si-O Bond Cleavage
}

Michael R. Gau, ${ }^{\dagger}$ Michael J. Zdilla*

*Department of Chemistry, Temple University, 1901 N. 13th St., Philadelphia, PA 19122

'Department of Chemistry, University of Pennsylvania, 231 S. 34 Street, Philadelphia, PA 19104-6323 


\section{Contents}

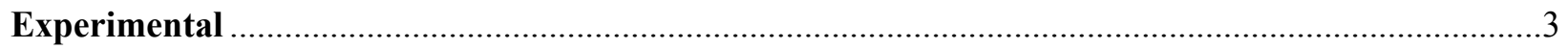

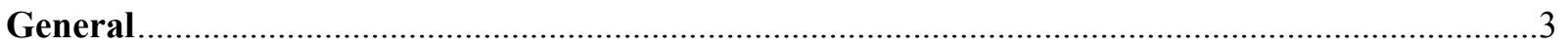

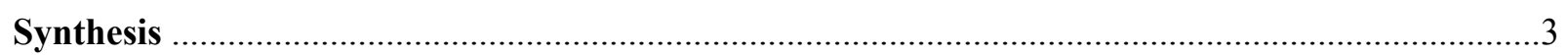

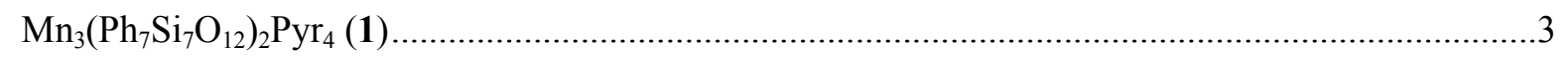

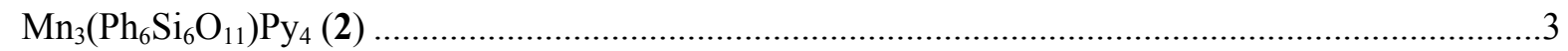

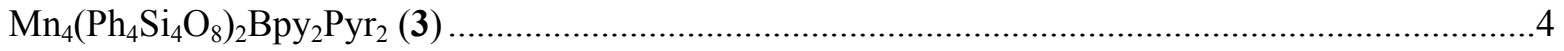

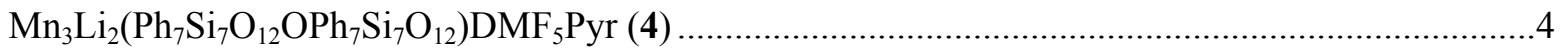

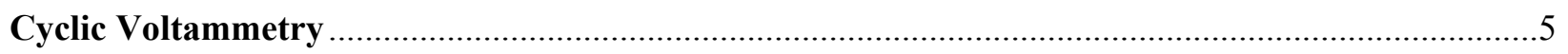

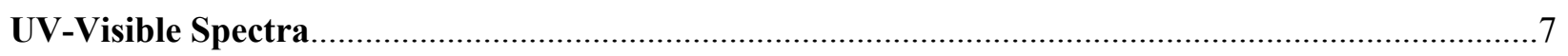

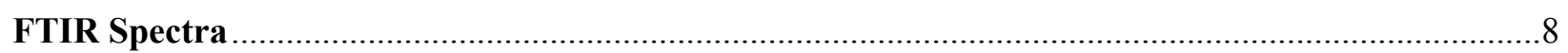

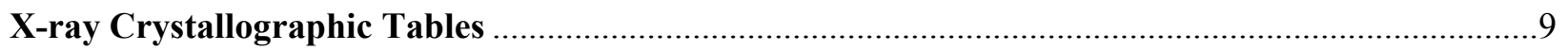

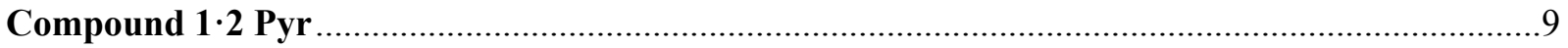

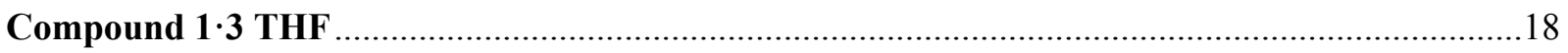

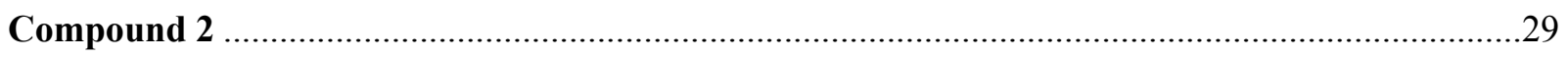

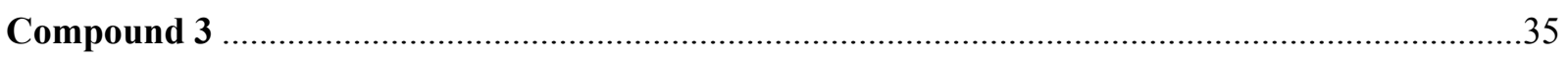

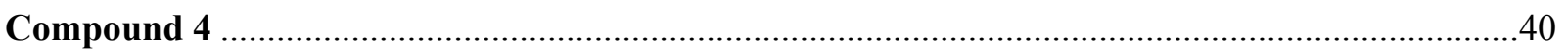

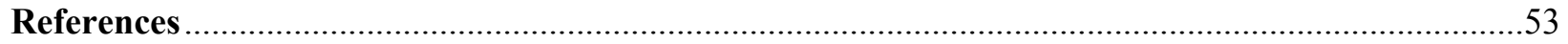




\section{Experimental}

\section{General}

All manipulations were performed under a dry, anaerobic $\mathrm{N}_{2}$ atmosphere using Schlenk and glovebox techniques. Reagents and solvents were purchased from commercial vendors (Aldrich, Strem, Fisher, Acros), were of highest available purity, and were used without further purification unless otherwise noted. Pentane, toluene, and dichloromethane (DCM) were purified using an Innovative Technology, Inc. Pure Solv system and stored in the glovebox over activated 4- $\AA$ molecular sieves for at least $24 \mathrm{~h}$ before use. Tetrahydrofuran (THF), ether $\left(\mathrm{Et}_{2} \mathrm{O}\right)$, and pyridine were distilled from sodium benzophenone ketyl under a nitrogen atmosphere and stored in the glovebox over 4- $\AA$ molecular sieves for $10 \mathrm{~h}$. Dimethylformamide (DMF) was distilled from calcium hydride under a nitrogen atmosphere and stored in the glovebox over $4-\AA$ molecular sieves for $24 \mathrm{~h}$. $\mathrm{Ph}_{7} \mathrm{Si}_{7} \mathrm{O}_{9}(\mathrm{OH})_{3}\left((\mathrm{~L} 1) \mathrm{H}_{3}\right)$ and $\mathrm{Ph}_{4} \mathrm{Si}_{4} \mathrm{O}_{4}(\mathrm{OH})_{4}\left((\mathrm{~L} 2) \mathrm{H}_{4}\right)$ were obtained from Hybrid Plastics Inc. The synthesis of $\mathrm{Mn}\left[\mathrm{N}\left(\mathrm{SiMe}_{3}\right)_{2}\right]_{2}$ was performed according to a published protocol. ${ }^{1} \mathrm{UV} /$ visible absorption spectra were acquired on a Shimadzu 2540 or 1800 spectrometer. Crystal diffraction data for all compounds were collected using a Bruker Kappa APEX II DUO diffractometer using Mo K $\alpha$ radiation $(\lambda=0.71073)$ from a fine-focused sealed tube. Data was collected at $100 \mathrm{~K}$ and structures were solved using direct or intrinsic phasing methods, and refined using the ShelXL package. ${ }^{2}$ Further crystallographic information is available in the crystallographic appendix. Elemental analyses were carried out under inert atmosphere by Bill Brennessel at the University of Rochester's CENTC Elemental Analysis Facility (Rochester, NY). Electrochemical studies were performed in anaerobic conditions using a standard three-electrode assembly: glassy carbon working, $\mathrm{Pt}$ wire auxiliary, and an $\mathrm{Ag} / \mathrm{AgCl}$ pseudo-reference were employed. Electrode potentials were corrected to Fc, which was used as an internal standard. The tetrabutylammonium tetrakis(pentafluorophenyl)borate was synthesized according to a published protocol. ${ }^{3}$

\section{Synthesis}

$\mathrm{Mn}_{3}\left(\mathrm{Ph}_{7} \mathrm{Si}_{7} \mathrm{O}_{12}\right)_{2} \mathrm{Pyr}_{4}(1)$

To a solution of $\mathrm{Mn}\left(\mathrm{NR}_{2}\right)_{2} \mathrm{THF}_{2}\left(\mathrm{R}=\mathrm{SiMe}_{3}\right)(201 \mathrm{mg}, 0.3865 \mathrm{mmol})$ in $1 \mathrm{~mL}$ of pyridine and 1 $\mathrm{mL}$ of toluene was added a solution of $(\mathrm{L} 1) \mathrm{H}_{3}(235 \mathrm{mg}, 0.2523 \mathrm{mmol})$ in $1 \mathrm{~mL}$ of pyridine and 1 $\mathrm{mL}$ of toluene. $3 \mathrm{~mL}$ of pentane was added to the resulting solution and the mixture placed in the freezer at $-40{ }^{\circ} \mathrm{C}$ for 72 hours. The clear white crystals were identified using single crystal X-ray diffraction, washed 3 times with $\sim 3 \mathrm{~mL}$ of pentane, and dried in vacuo. Yield: $218 \mathrm{mg}(0.0923$ mmol, 73.2\%). Anal. Calcd for $\mathrm{Mn}_{3}\left(\mathrm{Ph}_{7} \mathrm{Si}_{7} \mathrm{O}_{12}\right)_{2} \mathrm{Pyr}_{4} \cdot 2$ Pyr: C, 53.671\%; H, 3.880\%; N, 2.568\%. Found: C, 53.680\%; H, 3.887\%; N, 2.517\%. UV/vis (THF) $\lambda_{\max } / \mathrm{nm}: 320$. IR (KBr) $\lambda_{\max } / \mathrm{cm}^{-1}$ : 3073, 3047 (m, aromatic C-H stretch); 2919, 2850 (w, C-H stretch), 1957, 1887, 1821 (w, overtone-combination bands); 1600, 1487, 1430 (m, C-C, C-N ring stretching); 1218, (m, C-H in plane bend); 1100-850 (m, Si-O-Si); 738, 694 (w, C-H aromatic out-of-plane bend).

$\mathrm{Mn}_{3}\left(\mathrm{Ph}_{6} \mathrm{Si}_{6} \mathrm{O}_{11}\right) \mathrm{Py}_{4}(2)$

A pressure flask was charged with a solution of 1 in $6 \mathrm{~mL}$ of pyridine $(175 \mathrm{mg})$ and a magnetic stir bar. The flask was degassed, placed under vacuum and an $\mathrm{O}_{2}$ balloon was used for the addition and allowed to stir at room temperature for 2-3 hours. The flask was degassed and brought into the $\mathrm{N}_{2}$ glove box. A Teflon screw cap was used to close the flask and the resulting solution was heated at $90-95{ }^{\circ} \mathrm{C}$ for 24 hours. The solution was allowed to cool to room 
temperature and dried in vacuo, rinsed with pentane and extracted with $4 \mathrm{~mL}$ THF. The THF solution was filtered and the filtrate layered with $6 \mathrm{~mL}$ of pentane and placed in the freezer at -35 ${ }^{\circ} \mathrm{C}$ for 48 hours. Pink/purple crystals were formed and identified by SC-XRD. The supernatant was decanted, the pink/purple crystals were washed twice with $\sim 2 \mathrm{~mL}$ of cold THF, and 2-3 times with $2 \mathrm{~mL}$ of ether and $2 \mathrm{~mL}$ of pentane and dried in vacuo. Yield: $30 \mathrm{mg}(0.01432 \mathrm{mmol}$, $19.3 \%$ ). UV/vis (THF) $\lambda_{\max } / \mathrm{nm}: 572$, 438. IR (KBr) $\lambda_{\max } / \mathrm{cm}^{-1}: 3070,3050(\mathrm{w}$, aromatic C-H stretch); 2920, 2850 (w, C-H stretch), 1960, 1890, 1820 (w, overtone-combination bands); 1600, 1480, 1450, 1430 (m, C-C, C-N ring stretching); 1210 (m, C-H in plane bend); 1124 (m, Si-O); 1000-850 (m, Si-O-Si); 742, 697 (w, C-H aromatic out-of-plane bend).

$\mathrm{Mn}_{4}\left(\mathrm{Ph}_{4} \mathrm{Si}_{4} \mathrm{O}_{8}\right)_{2} \mathrm{Bpy}_{2} \mathrm{Pyr}_{2}(3)$

To a solution of $\mathrm{Mn}\left(\mathrm{NR}_{2}\right)_{2}(\mathrm{THF})_{2}(48 \mathrm{mg}, 0.0923 \mathrm{mmol})$ in $1 \mathrm{~mL}$ of pyridine was added a solution of 2,2'-bipyridine ( $7 \mathrm{mg}, 0.0448 \mathrm{mmol})$ in $1 \mathrm{~mL}$ of pyridine. A solution of $(\mathrm{L} 2) \mathrm{H}_{4}(25$ $\mathrm{mg}, 0.0453 \mathrm{mmol}$ ) in $1 \mathrm{~mL}$ of $N, N$-dimethylformamide was then added to the red/orange $\mathrm{Mn}\left(\mathrm{NR}_{2}\right)$ Bpy solution. The resulting yellow solution was placed in a double vial apparatus with diethylether at $-30^{\circ} \mathrm{C}$. Yellow block crystals were formed and identified using single crystal Xray diffraction. The crystals were washed 3 times with $\sim 3 \mathrm{~mL}$ of pentane and dried in vacuo. Yield: $35 \mathrm{mg}(0.0178 \mathrm{mmol}, 77.8 \%)$. Anal. Calcd for $\mathrm{Mn}_{4}\left(\mathrm{Ph}_{4} \mathrm{Si}_{4} \mathrm{O}_{8}\right)_{2}(\mathrm{Bpy})_{2}(\mathrm{Pyr})_{2} \cdot 2 \mathrm{DMF}$ : C, $52.067 \%$; H, 4.237\%; N, 6.039\%. Found: C, 51.691\%; H, 4.283\%; N, 6.187\%. UV/vis (DMF suspension) $\lambda_{\max } / \mathrm{nm}: 336$. IR $(\mathrm{KBr}) \lambda_{\max } / \mathrm{cm}^{-1}: 3064-3000$ (w, aromatic C-H stretch); 2925, 2848 (w, C-H stretch), 1950, 1880, 1850 (w, overtone-combination bands), 1670 ( $\mathrm{C}=\mathrm{O}$ stretching); 1600, 1470, 1440 (m, C-C, C-N ring stretching); 1383 (w, C-H bend); 1310, 1250, 1215 (m, C-H in plane bend); 1124 (m, Si-O); 1000-850 (m, Si-O-Si); 743, 701 (w, C-H aromatic out-of-plane bend).

$\mathrm{Mn}_{3} \mathrm{Li}_{2}\left(\mathrm{Ph}_{7} \mathrm{Si}_{7} \mathrm{O}_{12} \mathrm{OPh}_{7} \mathrm{Si}_{7} \mathrm{O}_{12}\right) \mathrm{DMF}_{5} \mathrm{Pyr}(4)$

A suspension of LiOH ( $2 \mathrm{mg}, 0.0835 \mathrm{mmol})$ in $2 \mathrm{~mL}$ of DMF was stirred vigorously at room temperature for 24 hours. To a solution of $\mathrm{Mn}\left(\mathrm{NR}_{2}\right)_{2}(94 \mathrm{mg}, 0.250 \mathrm{mmol})$ in $1 \mathrm{~mL}$ of pyridine and $1 \mathrm{~mL}$ of toluene was added a solution of $(\mathrm{L1}) \mathrm{H}_{3}(156 \mathrm{mg}, 0.1675 \mathrm{mmol})$ in $1 \mathrm{~mL}$ of pyridine. The in situ complex 1 was then added to the stirring solution of $\mathrm{LiOH}$ and immediately after, a solution of n-butyllithium $(33.4 \mu \mathrm{L}$ of $2.5 \mathrm{M}$ in hexanes, $0.0835 \mathrm{mmol})$ in $2 \mathrm{~mL}$ of ether was added. The solution stirred for 2 hours at room temperature. It was then filtered and layered with $6 \mathrm{~mL}$ ether and $4 \mathrm{~mL}$ pentane, and placed in the freezer at $-35^{\circ} \mathrm{C}$ for 48 hours. Clear block crystals were formed and identified using SC-XRD. To further precipitate complex 4, an additional $6 \mathrm{~mL}$ of ether and $4 \mathrm{~mL}$ of pentane was added and the solution was replaced in the freezer at $-35^{\circ} \mathrm{C}$ for an additional 48 hours. The crystals were washed 3 times with $\sim 3 \mathrm{~mL}$ of pentane and dried in vacuo. Yield: $142 \mathrm{mg}(0.0749 \mathrm{mmol}, 65.1 \%)$. Anal. Calcd for $\mathrm{Mn}_{3} \mathrm{Li}_{2}\left(\mathrm{Ph}_{7} \mathrm{Si}_{7} \mathrm{O}_{12} \mathrm{OPh}_{7} \mathrm{Si}_{7} \mathrm{O}_{12}\right) \mathrm{DMF}_{5} \mathrm{Pyr} \cdot \mathrm{Pyr} / 0.5 \mathrm{C}_{5} \mathrm{H}_{12}: \mathrm{C}, 51.289 \%$; H, 4.670\%; N, 3.755\%. Found: C, 51.532\%; H, 4.369\%; N, 3.504\%. UV/vis (DMF) $\lambda_{\max } / \mathrm{nm}: 336$. IR (KBr) $\lambda_{\max } / \mathrm{cm}^{-1}$ : 3070, 3040, 3000 (w, aromatic C-H stretch); 2930, 2890 (m, C-H stretch from $\mathrm{CH}_{3}$ ), 1960, 1890, 1850 (w, overtone bands), 1670 ( $\mathrm{C}=\mathrm{O}$ stretching); 1590, 1490, 1430 (m, C-C, C-N ring stretching); 1390 (w, C-H bend); 1260, (m, C-H in plane bend); 1200-850 (m, Si-O-Si); 738, 700 (w, C-H aromatic out-of-plane bend). 


\section{UV/visible Spectra of Complexes before and after $\mathrm{O}_{2}$ Addition.}

$6 \mathrm{mg}$ of 1 was dissolved in $6 \mathrm{~mL}$ of THF. An aliquot was taken and a UV/visible spectrum was taken. The resulting solution was placed in a $10 \mathrm{~mL}$ round bottom flask and degassed. $\mathrm{An} \mathrm{O}_{2}$ balloon was inserted and the reaction was allowed to stir at room temperature for 3 hours. An aliquot of the colored solution (2) was measured in the UV/visible spectrometer. The procedure was repeated for 3 ( $4 \mathrm{mg}$ in $5 \mathrm{~mL}$ of DMF), and 4 (2 $\mathrm{mg}$ in $5 \mathrm{~mL}$ of DMF), albeit 3 did not fully dissolve in DMF and resulted in a suspension.

\section{Cyclic Voltammetry}

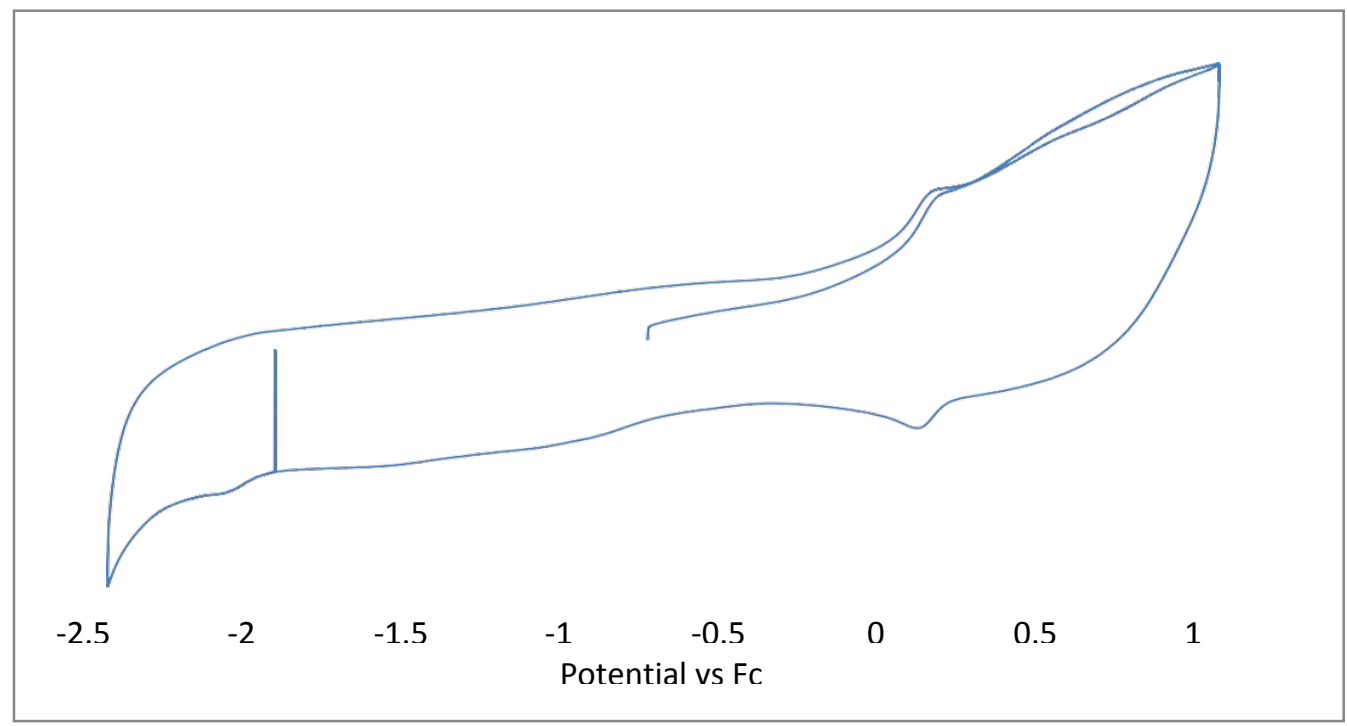

Figure S1. CV of $1 \mathrm{mM}$ complex 1 in THF 0.1 M TBABArF. OCP: -0.74 V. Scan rate:100 $\mathrm{mV} / \mathrm{s}$. Sweep direction: Positive.

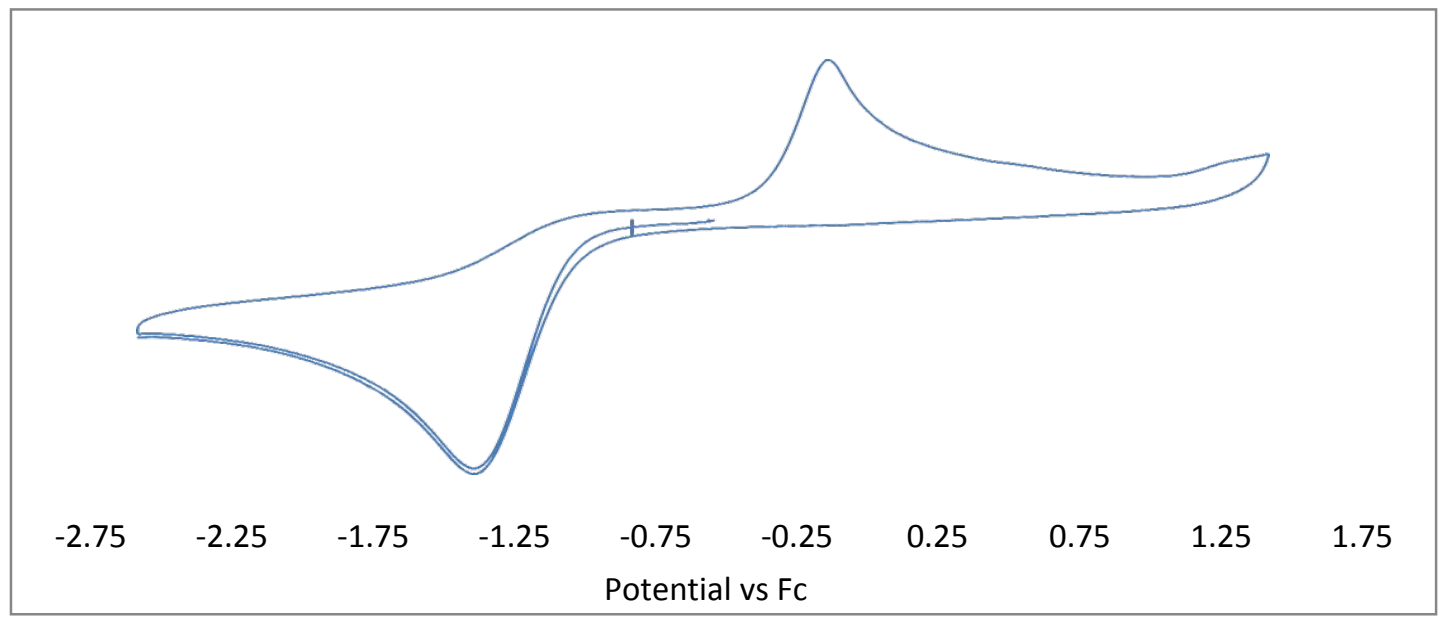

Figure S2. CV of $1 \mathrm{mM} 2$ in $0.05 \mathrm{M}$ TBA BArF in THF. OCP: $-0.543 \mathrm{~V}$. Scan rate: $100 \mathrm{mV} / \mathrm{s}$. Sweep direction: Negative. 


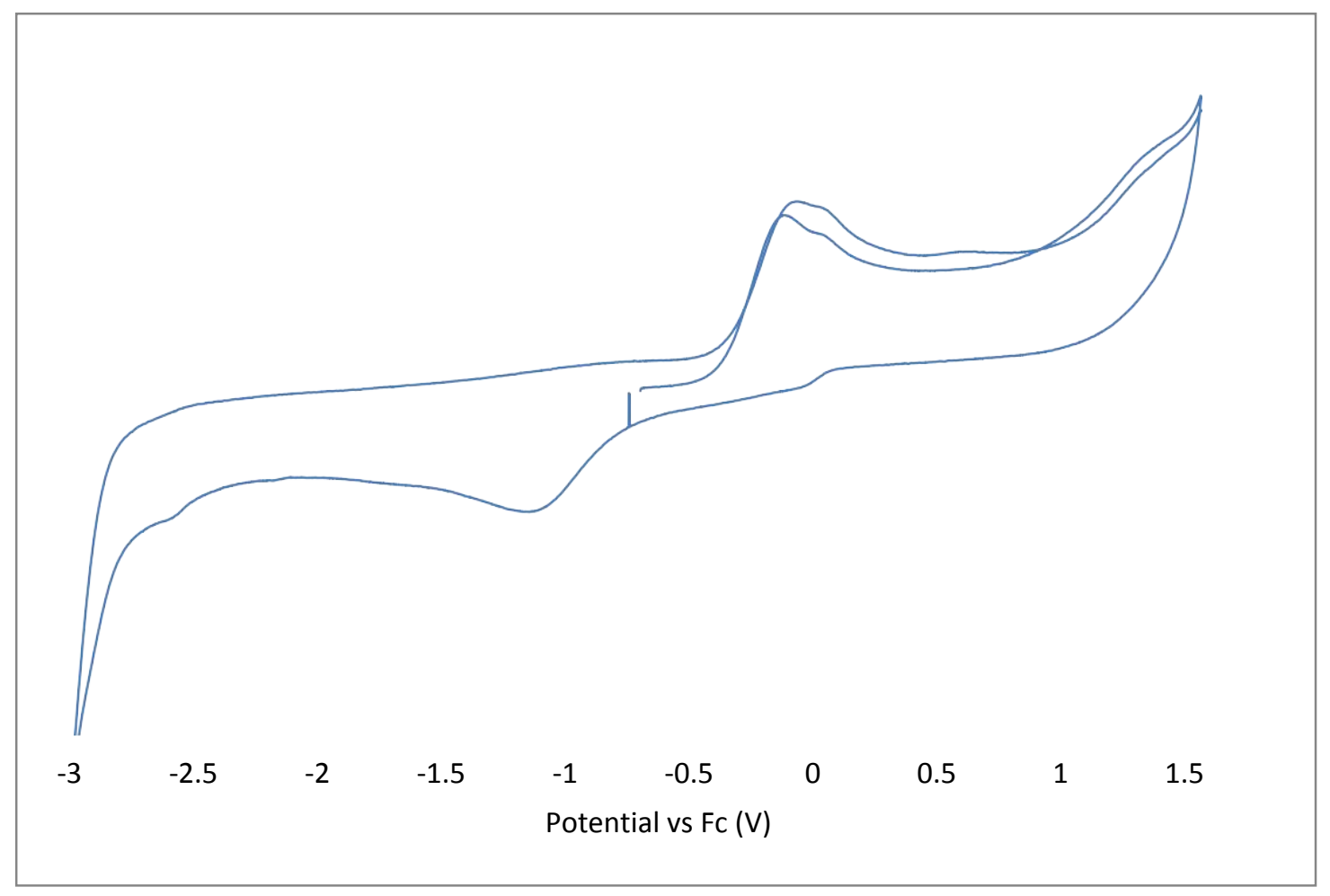

Figure S3. CV of $1 \mathrm{mM} 4$ in $0.05 \mathrm{M}$ TBA BAr ${ }^{\mathrm{F}}$ in THF. OCP: $-0.701 \mathrm{~V}$. Scan rate: 100 $\mathrm{mV} / \mathrm{s}$. Sweep direction: Positive. When the potential was swept in the negative direction first, no reduction half-wave at $-1.1785 \mathrm{~V}$ vs Fc could be seen, 


\section{UV-Visible Spectra}

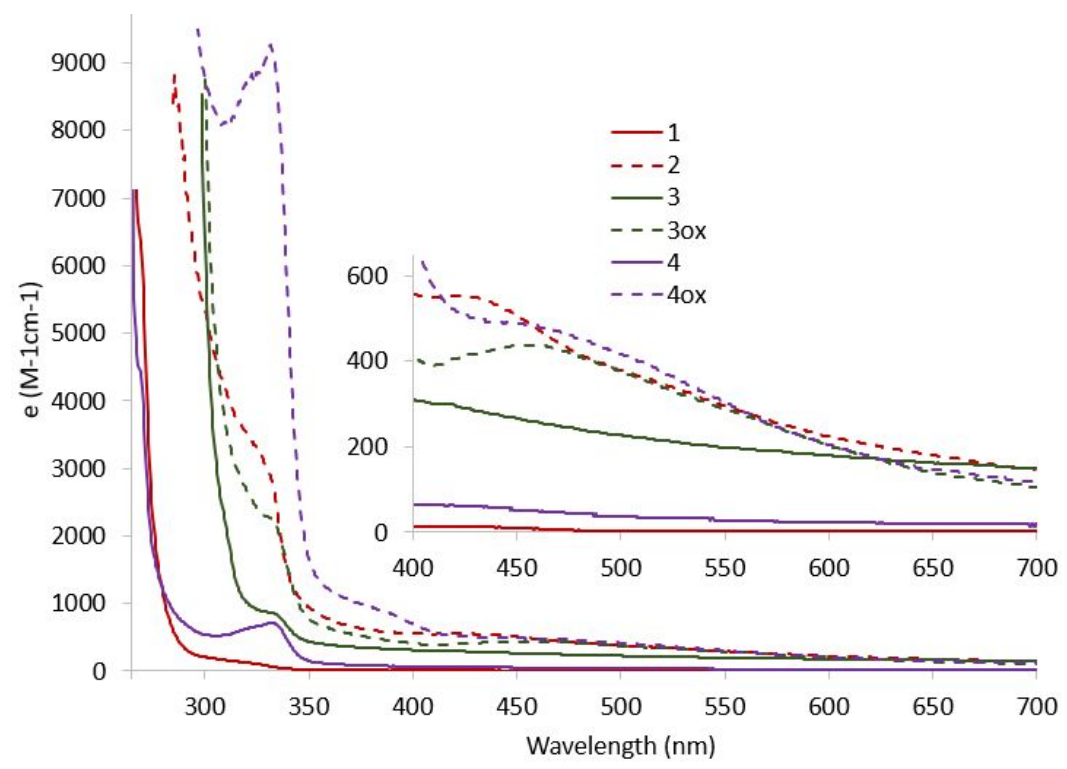

Figure S4. UV-Visible Spectra of 1-4 as well as the products of $\mathrm{O}_{2}$ oxidized 3 and $\mathbf{4}$ (note that 2 is the oxidized product of $\mathbf{1}$ ). 


\section{FTIR Spectra}

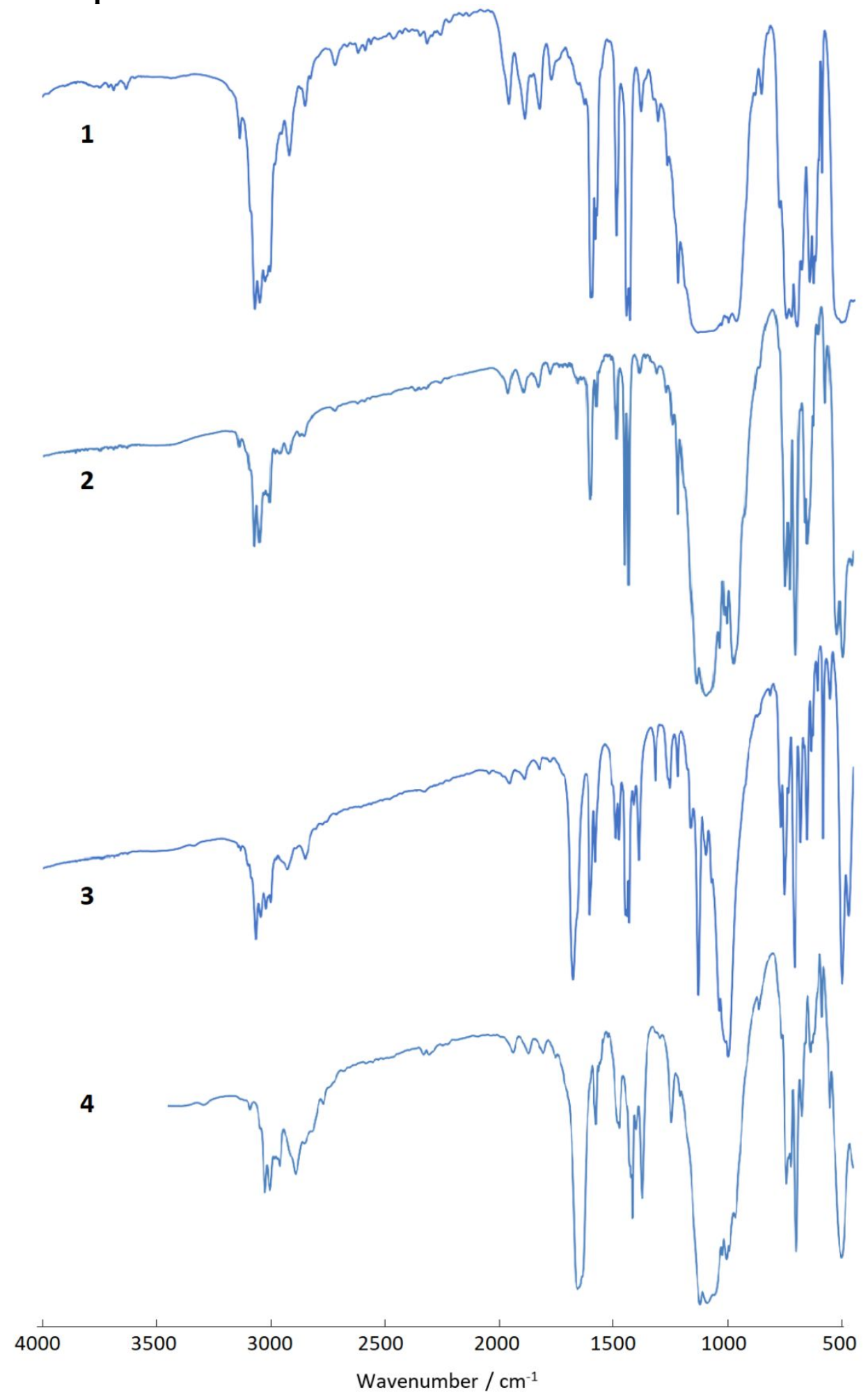

Figure S5. Transmission FTIR Spectra of 1-4 suspended in $\mathrm{KBr}$. 


\section{X-ray Crystallographic Tables}

\section{Compound 1.2 Pyr}

Table S1. Crystal data and structure refinement for $1 \cdot 2$ Pyr.

\begin{tabular}{|c|c|}
\hline Identification code & $1 \cdot 2$ Pyr \\
\hline Empirical formula & $\mathrm{C}_{114} \mathrm{H}_{100} \mathrm{Mn}_{3} \mathrm{~N}_{6} \mathrm{O}_{24} \mathrm{Si}_{14}$ \\
\hline Formula weight & 2496.07 \\
\hline Temperature/K & $100(2)$ \\
\hline Crystal system & triclinic \\
\hline Space group & P-1 \\
\hline $\mathrm{a} / \AA$ & $14.987(3)$ \\
\hline $\mathrm{b} / \AA$ & $16.351(4)$ \\
\hline $\mathrm{c} / \AA \AA$ & $29.571(6)$ \\
\hline$\alpha /^{\circ}$ & $76.487(4)$ \\
\hline$\beta /{ }^{\circ}$ & $82.781(4)$ \\
\hline$\gamma /{ }^{\circ}$ & $72.809(4)$ \\
\hline Volume $/ \AA^{3}$ & $6718(2)$ \\
\hline $\mathrm{Z}$ & 2 \\
\hline$\rho_{\text {calc }} \mathrm{g} / \mathrm{cm}^{3}$ & 1.234 \\
\hline$\mu / \mathrm{mm}^{-1}$ & 0.467 \\
\hline $\mathrm{F}(000)$ & 2578.0 \\
\hline Crystal size $/ \mathrm{mm}^{3}$ & $0.718 \times 0.340 \times 0.145$ \\
\hline Radiation & $\operatorname{MoK} \alpha(\lambda=0.71073)$ \\
\hline $2 \Theta$ range for data collection $/{ }^{\circ}$ & 2.666 to 55.89 \\
\hline Index ranges & $-19 \leq \mathrm{h} \leq 19,-21 \leq \mathrm{k} \leq 21,-38 \leq 1 \leq 38$ \\
\hline Reflections collected & 103688 \\
\hline Independent reflections & $31955\left[\mathrm{R}_{\mathrm{int}}=0.0605, \mathrm{R}_{\text {sigma }}=0.0751\right]$ \\
\hline Data/restraints/parameters & $31955 / 394 / 1546$ \\
\hline Goodness-of-fit on $\mathrm{F}^{2}$ & 1.026 \\
\hline Final $R$ indexes $[\mathrm{I}>=2 \sigma(\mathrm{I})]$ & $\mathrm{R}_{1}=0.0739, \mathrm{wR}_{2}=0.1893$ \\
\hline Final R indexes [all data] & $\mathrm{R}_{1}=0.1254, \mathrm{wR}_{2}=0.2292$ \\
\hline Largest diff. peak/hole / e $\AA^{-3}$ & $1.61 /-0.76$ \\
\hline
\end{tabular}

Table S2. Bond Lengths for $1 \cdot 2$ Pyr.

\begin{tabular}{llrlrr}
\multicolumn{1}{c}{ Atom } & Atom & Length $/ \AA$ & \multicolumn{1}{c}{ Atom } & Atom & Length/ \\
\hline $\mathrm{Mn} 1$ & $\mathrm{O} 12$ & $2.042(3)$ & $\mathrm{C} 19$ & $\mathrm{C} 24$ & $1.394(7)$ \\
$\mathrm{Mn} 1$ & $\mathrm{O} 16$ & $2.141(3)$ & $\mathrm{C} 20$ & $\mathrm{C} 21$ & $1.387(7)$ \\
$\mathrm{Mn} 1$ & $\mathrm{O} 24$ & $2.207(3)$ & $\mathrm{C} 21$ & $\mathrm{C} 22$ & $1.345(9)$ \\
$\mathrm{Mn} 1$ & $\mathrm{~N} 1$ & $2.261(4)$ & $\mathrm{C} 22$ & $\mathrm{C} 23$ & $1.348(10)$ \\
$\mathrm{Mn} 1$ & $\mathrm{~N} 2$ & $2.296(4)$ & $\mathrm{C} 23$ & $\mathrm{C} 24$ & $1.409(9)$ \\
$\mathrm{Mn} 2$ & $\mathrm{O} 13$ & $1.982(3)$ & $\mathrm{C} 25$ & $\mathrm{C} 26$ & $1.320(13)$ \\
$\mathrm{Mn} 2$ & $\mathrm{O} 1$ & $2.067(3)$ & $\mathrm{C} 25$ & $\mathrm{C} 30$ & $1.367(12)$
\end{tabular}




\begin{tabular}{|c|c|c|c|c|c|}
\hline \multicolumn{2}{|c|}{ Atom } & \multirow{2}{*}{$\frac{\text { Length } / \AA}{2.124(3)}$} & \multicolumn{2}{|c|}{ Atom } & \multirow{2}{*}{$\frac{\text { Length/Å }}{1.422(11)}$} \\
\hline$\overline{\mathrm{Mn} 2}$ & O16 & & C26 & $\mathrm{C} 27$ & \\
\hline $\mathrm{Mn} 2$ & N3 & $2.226(4)$ & $\mathrm{C} 27$ & $\mathrm{C} 28$ & $1.278(13)$ \\
\hline $\mathrm{Mn} 2$ & Mn3 & $3.2337(11)$ & $\mathrm{C} 28$ & $\mathrm{C} 29$ & $1.308(14)$ \\
\hline Mn3 & O9 & $1.987(3)$ & $\mathrm{C} 29$ & $\mathrm{C} 30$ & $1.490(12)$ \\
\hline $\mathrm{Mn} 3$ & $\mathrm{O} 24$ & $2.115(3)$ & C31A & C36A & $1.370(17)$ \\
\hline $\mathrm{Mn} 3$ & $\mathrm{O} 1$ & $2.124(3)$ & $\mathrm{C} 31 \mathrm{~A}$ & C32 & $1.373(13)$ \\
\hline Mn3 & N4 & $2.332(4)$ & C34 & $\mathrm{C} 35$ & 1.3900 \\
\hline Mn3 & $\mathrm{O} 12$ & $2.366(3)$ & C34 & C33 & 1.3900 \\
\hline Sil & $\mathrm{O} 1$ & $1.599(3)$ & $\mathrm{C} 35$ & C36 & 1.3900 \\
\hline Sil & $\mathrm{O} 2$ & $1.643(3)$ & C36 & C31 & 1.3900 \\
\hline Sil & $\mathrm{O} 3$ & $1.655(3)$ & C31 & C32 & 1.3900 \\
\hline Sil & $\mathrm{C} 1$ & $1.860(4)$ & C32 & $\mathrm{C} 33$ & 1.3900 \\
\hline $\mathrm{Si} 2$ & $\mathrm{O} 11$ & $1.618(3)$ & C33 & C34A & $1.401(19)$ \\
\hline $\mathrm{Si} 2$ & $\mathrm{O} 2$ & $1.625(3)$ & C34A & C35A & $1.36(2)$ \\
\hline $\mathrm{Si} 2$ & O5 & $1.631(3)$ & $\mathrm{C} 35 \mathrm{~A}$ & $\mathrm{C} 36 \mathrm{~A}$ & $1.427(18)$ \\
\hline $\mathrm{Si} 2$ & $\mathrm{C} 7$ & $1.848(5)$ & C35A & $\mathrm{C} 35 \mathrm{~A}^{1}$ & $1.81(4)$ \\
\hline $\mathrm{Si} 3$ & O5 & $1.621(4)$ & C37 & C42 & $1.392(7)$ \\
\hline $\mathrm{Si} 3$ & O4 & $1.630(4)$ & C37 & C38 & $1.393(8)$ \\
\hline $\mathrm{Si} 3$ & $\mathrm{O} 8$ & $1.637(5)$ & C38 & C39 & $1.398(9)$ \\
\hline $\mathrm{Si} 3$ & $\mathrm{C} 13$ & $1.858(5)$ & C39 & $\mathrm{C} 40$ & $1.362(13)$ \\
\hline $\mathrm{Si} 4$ & O6 & $1.605(4)$ & $\mathrm{C} 40$ & C41 & $1.341(12)$ \\
\hline $\mathrm{Si} 4$ & $\mathrm{O} 3$ & $1.626(3)$ & C41 & $\mathrm{C} 42$ & $1.396(8)$ \\
\hline $\mathrm{Si} 4$ & O4 & $1.633(4)$ & $\mathrm{C} 43$ & $\mathrm{C} 48$ & $1.359(8)$ \\
\hline $\mathrm{Si} 4$ & C19 & $1.856(5)$ & $\mathrm{C} 43$ & $\mathrm{C} 44$ & $1.367(8)$ \\
\hline Si5 & O9 & $1.567(4)$ & $\mathrm{C} 44$ & $\mathrm{C} 45$ & $1.406(8)$ \\
\hline $\mathrm{Si} 5$ & $\mathrm{O} 7$ & $1.659(4)$ & $\mathrm{C} 45$ & $\mathrm{C} 46$ & $1.330(10)$ \\
\hline $\mathrm{Si} 5$ & O6 & $1.660(4)$ & $\mathrm{C} 46$ & C47 & $1.367(10)$ \\
\hline $\mathrm{Si} 5$ & $\mathrm{C} 25$ & $1.859(7)$ & $\mathrm{C} 47$ & $\mathrm{C} 48$ & $1.408(8)$ \\
\hline $\mathrm{Si} 6$ & $\mathrm{O} 10$ & $1.605(3)$ & C49 & C54 & $1.382(8)$ \\
\hline $\mathrm{Si} 6$ & $\mathrm{O} 7$ & $1.606(4)$ & C49 & C50 & $1.415(7)$ \\
\hline $\mathrm{Si} 6$ & O8 & $1.616(5)$ & $\mathrm{C} 50$ & C51 & $1.396(9)$ \\
\hline $\mathrm{Si} 6$ & C31 & $1.893(5)$ & C51 & C52 & $1.349(10)$ \\
\hline Si6 & C31A & $1.895(11)$ & C52 & C53 & $1.373(10)$ \\
\hline $\mathrm{Si} 7$ & O12 & $1.597(3)$ & C53 & C54 & $1.381(9)$ \\
\hline $\mathrm{Si} 7$ & $\mathrm{O} 10$ & $1.635(3)$ & C55 & $\mathrm{C} 60$ & $1.379(7)$ \\
\hline $\mathrm{Si} 7$ & $\mathrm{O} 11$ & $1.636(3)$ & C55 & C56 & $1.384(7)$ \\
\hline $\mathrm{Si} 7$ & $\mathrm{C} 37$ & $1.870(5)$ & C56 & C57 & $1.379(9)$ \\
\hline $\mathrm{Si} 8$ & $\mathrm{O} 13$ & $1.572(3)$ & C57 & C58 & $1.368(10)$ \\
\hline $\mathrm{Si} 8$ & $\mathrm{O} 17$ & $1.652(4)$ & C58 & C59 & $1.362(9)$ \\
\hline Si8 & $\mathrm{O} 14$ & $1.654(4)$ & C59 & C60 & $1.376(8)$ \\
\hline $\mathrm{Si} 8$ & $\mathrm{C} 43$ & $1.876(4)$ & C61 & C66 & $1.367(7)$ \\
\hline
\end{tabular}




\begin{tabular}{|c|c|c|c|c|c|}
\hline \multicolumn{2}{|c|}{ Atom } & \multicolumn{2}{|l|}{ Length/Å } & \multirow[t]{2}{*}{ Atom } & \multirow{2}{*}{$\frac{\text { Length/Å }}{1.382(7)}$} \\
\hline Si9 & $\mathrm{O} 15$ & $1.621(3)$ & C61 & & \\
\hline $\mathrm{Si} 9$ & O14 & $1.623(3)$ & C62 & C63 & $1.404(7)$ \\
\hline $\mathrm{Si} 9$ & O19 & $1.634(3)$ & C63 & C64 & $1.364(9)$ \\
\hline Si9 & C49 & $1.849(5)$ & C64 & C65 & $1.371(10)$ \\
\hline Sil0 & O16 & $1.603(3)$ & C65 & C66 & $1.397(8)$ \\
\hline Si10 & O15 & $1.637(3)$ & C67A & C68A & $1.300(9)$ \\
\hline Sil0 & $\mathrm{O} 21$ & $1.645(3)$ & C67A & C72 & $1.483(11)$ \\
\hline Silo & C55 & $1.851(5)$ & C68A & C69A & $1.389(9)$ \\
\hline Sil1 & $\mathrm{O} 21$ & $1.613(3)$ & C69A & C70A & $1.250(12)$ \\
\hline Sil1 & $\mathrm{O} 22$ & $1.628(3)$ & C70A & C71 & $1.383(13)$ \\
\hline Si11 & $\mathrm{O} 20$ & $1.629(3)$ & C71 & C72 & $1.399(10)$ \\
\hline Sil1 & C61 & $1.861(4)$ & C73 & $\mathrm{C} 74$ & $1.239(19)$ \\
\hline Si12 & O19 & $1.604(4)$ & C73 & C78A & $1.35(2)$ \\
\hline Si12 & O18 & $1.622(4)$ & C73 & C78 & $1.451(15)$ \\
\hline Si12 & $\mathrm{O} 20$ & $1.628(3)$ & C73 & C74A & $1.57(3)$ \\
\hline Si12 & C67A & $1.846(6)$ & C74 & C75 & $1.423(19)$ \\
\hline Si13 & O17 & $1.616(3)$ & C74A & C75A & $1.36(3)$ \\
\hline Si13 & $\mathrm{O} 23$ & $1.617(3)$ & C75 & C76 & $1.354(17)$ \\
\hline Si13 & O18 & $1.628(4)$ & $\mathrm{C} 75 \mathrm{~A}$ & C76A & $1.39(2)$ \\
\hline Si13 & $\mathrm{C} 73$ & $1.846(6)$ & C76 & C77 & $1.390(17)$ \\
\hline Si14 & $\mathrm{O} 24$ & $1.603(3)$ & C76A & C77A & $1.40(3)$ \\
\hline Si14 & $\mathrm{O} 23$ & $1.623(3)$ & C77 & C78 & $1.377(15)$ \\
\hline Sil4 & $\mathrm{O} 22$ & $1.646(3)$ & C77A & C78A & $1.35(2)$ \\
\hline Si14 & C79 & $1.859(5)$ & C79 & $\mathrm{C} 80$ & $1.388(9)$ \\
\hline N1 & C89 & $1.304(7)$ & C79 & C84 & $1.398(8)$ \\
\hline N1 & $\mathrm{C} 85$ & $1.339(8)$ & $\mathrm{C} 80$ & C81 & $1.392(10)$ \\
\hline N2 & $\mathrm{C} 90$ & $1.343(7)$ & C81 & $\mathrm{C} 82$ & $1.409(13)$ \\
\hline N2 & C94 & $1.348(7)$ & C82 & $\mathrm{C} 83$ & $1.349(13)$ \\
\hline N3 & C99 & $1.331(7)$ & $\mathrm{C} 83$ & C84 & $1.378(10)$ \\
\hline N3 & C95 & $1.348(7)$ & $\mathrm{C} 85$ & C86 & $1.387(11)$ \\
\hline N4 & C104 & $1.339(6)$ & $\mathrm{C} 86$ & C87 & $1.312(13)$ \\
\hline N4 & $\mathrm{C} 100$ & $1.343(7)$ & C87 & C88 & $1.359(12)$ \\
\hline $\mathrm{C} 1$ & C6 & $1.394(6)$ & $\mathrm{C} 88$ & C89 & $1.402(8)$ \\
\hline $\mathrm{C} 1$ & $\mathrm{C} 2$ & $1.395(6)$ & C90 & C91 & $1.378(8)$ \\
\hline $\mathrm{C} 2$ & C3 & $1.396(6)$ & C91 & C92 & $1.370(11)$ \\
\hline C3 & $\mathrm{C} 4$ & $1.373(8)$ & C92 & C93 & $1.372(12)$ \\
\hline $\mathrm{C} 4$ & $\mathrm{C} 5$ & $1.385(9)$ & C93 & C94 & $1.377(9)$ \\
\hline C5 & C6 & $1.389(7)$ & C95 & C96 & $1.363(9)$ \\
\hline $\mathrm{C} 7$ & $\mathrm{C} 12$ & $1.398(7)$ & C96 & C97 & $1.380(11)$ \\
\hline $\mathrm{C} 7$ & $\mathrm{C} 8$ & $1.402(7)$ & C97 & C98 & $1.362(10)$ \\
\hline $\mathrm{C} 8$ & C9 & $1.375(8)$ & C98 & C99 & $1.393(8)$ \\
\hline
\end{tabular}




\begin{tabular}{|c|c|c|c|c|c|}
\hline Atom & Atom & Length/Å & Atom & Atom & Length/Å \\
\hline C9 & $\mathrm{C} 10$ & $1.381(9)$ & $\mathrm{C} 100$ & C101 & $1.382(8)$ \\
\hline $\mathrm{C} 10$ & $\mathrm{C} 11$ & $1.394(9)$ & C101 & C102 & $1.366(10)$ \\
\hline C11 & $\mathrm{C} 12$ & $1.384(8)$ & C102 & C103 & $1.351(9)$ \\
\hline $\mathrm{C} 13$ & $\mathrm{C} 18$ & $1.343(11)$ & C103 & C104 & $1.388(7)$ \\
\hline C13 & $\mathrm{C} 14$ & $1.389(12)$ & N5 & C105 & $1.315(13)$ \\
\hline C13 & C18A & 1.3900 & N5 & C109 & $1.320(12)$ \\
\hline C13 & C14A & 1.3900 & C105 & C106 & $1.379(17)$ \\
\hline C18A & C17A & 1.3900 & C106 & C107 & $1.31(2)$ \\
\hline C17A & C16 & 1.3900 & C107 & C108 & $1.18(2)$ \\
\hline C16 & $\mathrm{C} 17$ & $1.311(12)$ & C108 & C109 & $1.412(17)$ \\
\hline $\mathrm{C} 16$ & $\mathrm{C} 15$ & $1.386(12)$ & N6 & C114 & $1.317(10)$ \\
\hline $\mathrm{C} 16$ & $\mathrm{C} 15 \mathrm{~A}$ & 1.3900 & N6 & C110 & $1.322(10)$ \\
\hline $\mathrm{C} 15 \mathrm{~A}$ & C14A & 1.3900 & C110 & C111 & $1.376(10)$ \\
\hline C14 & $\mathrm{C} 15$ & $1.395(12)$ & C111 & C112 & $1.375(11)$ \\
\hline $\mathrm{C} 17$ & $\mathrm{C} 18$ & $1.390(11)$ & C112 & C113 & $1.361(11)$ \\
\hline C19 & $\mathrm{C} 20$ & $1.381(7)$ & C113 & C114 & $1.334(11)$ \\
\hline
\end{tabular}

${ }^{1}-\mathrm{X}, 1-\mathrm{Y}, 2-\mathrm{Z}$

Table S3. Bond Angles for 1·2 Pyr.

\begin{tabular}{|c|c|c|c|c|c|c|c|}
\hline Atom & Atom & Atom & Angle/ ${ }^{\circ}$ & Atom & Atom & Atom & Angle/ ${ }^{\circ}$ \\
\hline $\mathrm{O} 12$ & Mn1 & O16 & $99.64(11)$ & $\mathrm{C} 2$ & $\mathrm{C} 1$ & Si1 & $123.4(3)$ \\
\hline O12 & Mn1 & $\mathrm{O} 24$ & $85.64(11)$ & $\mathrm{C} 1$ & $\mathrm{C} 2$ & $\mathrm{C} 3$ & $122.0(4)$ \\
\hline O16 & Mn1 & $\mathrm{O} 24$ & $82.48(11)$ & $\mathrm{C} 4$ & $\mathrm{C} 3$ & $\mathrm{C} 2$ & $119.4(5)$ \\
\hline $\mathrm{O} 12$ & Mn1 & N1 & $115.67(14)$ & $\mathrm{C} 3$ & $\mathrm{C} 4$ & $\mathrm{C} 5$ & $120.1(5)$ \\
\hline O16 & Mn1 & N1 & $94.60(14)$ & $\mathrm{C} 4$ & $\mathrm{C} 5$ & C6 & $120.1(5)$ \\
\hline $\mathrm{O} 24$ & Mn1 & N1 & $158.65(14)$ & $\mathrm{C} 5$ & C6 & $\mathrm{C} 1$ & $121.4(5)$ \\
\hline $\mathrm{O} 12$ & Mn1 & $\mathrm{N} 2$ & $96.33(14)$ & $\mathrm{C} 12$ & $\mathrm{C} 7$ & $\mathrm{C} 8$ & $116.2(5)$ \\
\hline O16 & Mn1 & N2 & $160.97(14)$ & $\mathrm{C} 12$ & $\mathrm{C} 7$ & $\mathrm{Si} 2$ & $121.4(4)$ \\
\hline $\mathrm{O} 24$ & Mn1 & N2 & $88.50(13)$ & $\mathrm{C} 8$ & $\mathrm{C} 7$ & $\mathrm{Si} 2$ & $122.4(4)$ \\
\hline N1 & Mn1 & N2 & $87.71(16)$ & C9 & $\mathrm{C} 8$ & C7 & $122.9(5)$ \\
\hline $\mathrm{O} 13$ & $\mathrm{Mn} 2$ & $\mathrm{O} 1$ & $102.98(11)$ & $\mathrm{C} 8$ & C9 & $\mathrm{C} 10$ & $119.4(5)$ \\
\hline $\mathrm{O} 13$ & $\mathrm{Mn} 2$ & 016 & $124.17(11)$ & C9 & $\mathrm{C} 10$ & C11 & $119.9(6)$ \\
\hline $\mathrm{O} 1$ & $\mathrm{Mn} 2$ & O16 & $128.85(10)$ & C12 & C11 & $\mathrm{C} 10$ & $119.7(6)$ \\
\hline O13 & $\mathrm{Mn} 2$ & N3 & $98.07(14)$ & $\mathrm{C} 11$ & C12 & $\mathrm{C} 7$ & $121.9(5)$ \\
\hline $\mathrm{O} 1$ & $\mathrm{Mn} 2$ & N3 & $97.38(12)$ & C18 & C13 & $\mathrm{C} 14$ & $113.9(8)$ \\
\hline O16 & $\mathrm{Mn} 2$ & N3 & $94.71(12)$ & C18A & C13 & C14A & 120.0 \\
\hline O13 & $\mathrm{Mn} 2$ & Mn3 & 111.99(10) & C18 & C13 & $\mathrm{Si} 3$ & $123.5(7)$ \\
\hline $\mathrm{O} 1$ & $\mathrm{Mn} 2$ & Mn3 & $40.16(7)$ & $\mathrm{C} 14$ & C13 & $\mathrm{Si} 3$ & $122.0(6)$ \\
\hline O16 & $\mathrm{Mn} 2$ & Mn3 & $98.59(7)$ & C18A & C13 & $\mathrm{Si} 3$ & $120.0(6)$ \\
\hline N3 & $\mathrm{Mn} 2$ & Mn3 & $131.15(10)$ & C14A & C13 & $\mathrm{Si} 3$ & $120.0(6)$ \\
\hline
\end{tabular}




\begin{tabular}{|c|c|c|c|c|c|c|c|}
\hline Atom & Atom & Atom & Angle $/^{\circ}$ & Atom & Atom & Atom & Angle $/^{\circ}$ \\
\hline O9 & Mn3 & $\mathrm{O} 24$ & $119.27(13)$ & C13 & $\mathrm{C} 18 \mathrm{~A}$ & $\mathrm{C} 17 \mathrm{~A}$ & 120.0 \\
\hline O9 & Mn3 & $\mathrm{O} 1$ & $146.72(13)$ & $\mathrm{C} 16$ & C17A & C18A & 120.0 \\
\hline $\mathrm{O} 24$ & Mn3 & O1 & $93.68(11)$ & $\mathrm{C} 17$ & $\mathrm{C} 16$ & $\mathrm{C} 15$ & $121.9(7)$ \\
\hline O9 & Mn3 & N4 & $93.67(14)$ & $\mathrm{C} 15 \mathrm{~A}$ & $\mathrm{C} 16$ & C17A & 120.0 \\
\hline $\mathrm{O} 24$ & Mn3 & N4 & $90.70(13)$ & $\mathrm{C} 16$ & $\mathrm{C} 15 \mathrm{~A}$ & C14A & 120.0 \\
\hline $\mathrm{O} 1$ & Mn3 & N4 & $90.25(12)$ & $\mathrm{C} 15 \mathrm{~A}$ & C14A & C13 & 120.0 \\
\hline O9 & Mn3 & $\mathrm{O} 12$ & $97.16(12)$ & C13 & $\mathrm{C} 14$ & $\mathrm{C} 15$ & $124.6(10)$ \\
\hline $\mathrm{O} 24$ & Mn3 & $\mathrm{O} 12$ & $80.12(11)$ & $\mathrm{C} 16$ & $\mathrm{C} 15$ & $\mathrm{C} 14$ & $115.4(11)$ \\
\hline $\mathrm{O} 1$ & Mn3 & $\mathrm{O} 12$ & $83.14(10)$ & $\mathrm{C} 16$ & $\mathrm{C} 17$ & $\mathrm{C} 18$ & $119.7(10)$ \\
\hline N4 & Mn3 & $\mathrm{O} 12$ & $168.28(13)$ & $\mathrm{C} 13$ & $\mathrm{C} 18$ & $\mathrm{C} 17$ & $123.4(11)$ \\
\hline O9 & Mn3 & $\mathrm{Mn} 2$ & $167.87(9)$ & $\mathrm{C} 20$ & C19 & $\mathrm{C} 24$ & $117.1(5)$ \\
\hline $\mathrm{O} 24$ & Mn3 & $\mathrm{Mn} 2$ & $55.56(8)$ & $\mathrm{C} 20$ & C19 & $\mathrm{Si} 4$ & $121.2(4)$ \\
\hline $\mathrm{O} 1$ & Mn3 & Mn2 & $38.87(7)$ & $\mathrm{C} 24$ & C19 & $\mathrm{Si} 4$ & $121.7(4)$ \\
\hline N4 & Mn3 & $\mathrm{Mn} 2$ & $97.26(10)$ & C19 & $\mathrm{C} 20$ & $\mathrm{C} 21$ & $121.6(5)$ \\
\hline $\mathrm{O} 12$ & Mn3 & Mn2 & $71.56(7)$ & $\mathrm{C} 22$ & $\mathrm{C} 21$ & $\mathrm{C} 20$ & $120.4(6)$ \\
\hline O1 & Sil & $\mathrm{O} 2$ & $114.42(15)$ & $\mathrm{C} 21$ & $\mathrm{C} 22$ & $\mathrm{C} 23$ & $120.3(6)$ \\
\hline $\mathrm{O} 1$ & Sil & $\mathrm{O} 3$ & $104.60(15)$ & $\mathrm{C} 22$ & $\mathrm{C} 23$ & $\mathrm{C} 24$ & $120.5(6)$ \\
\hline $\mathrm{O} 2$ & Sil & $\mathrm{O} 3$ & $105.97(16)$ & C19 & $\mathrm{C} 24$ & $\mathrm{C} 23$ & $120.0(6)$ \\
\hline $\mathrm{O} 1$ & Si1 & $\mathrm{C} 1$ & $115.14(17)$ & $\mathrm{C} 26$ & $\mathrm{C} 25$ & C30 & $115.3(8)$ \\
\hline $\mathrm{O} 2$ & Sil & $\mathrm{C} 1$ & $106.15(17)$ & $\mathrm{C} 26$ & $\mathrm{C} 25$ & $\mathrm{Si} 5$ & $121.8(7)$ \\
\hline $\mathrm{O} 3$ & Sil & $\mathrm{C} 1$ & $110.20(17)$ & $\mathrm{C} 30$ & $\mathrm{C} 25$ & $\mathrm{Si} 5$ & $122.9(8)$ \\
\hline 011 & $\mathrm{Si} 2$ & $\mathrm{O} 2$ & $110.01(16)$ & $\mathrm{C} 25$ & $\mathrm{C} 26$ & $\mathrm{C} 27$ & $124.9(10)$ \\
\hline O11 & $\mathrm{Si} 2$ & O5 & 109.2(2) & $\mathrm{C} 28$ & $\mathrm{C} 27$ & $\mathrm{C} 26$ & $120.7(12)$ \\
\hline $\mathrm{O} 2$ & $\mathrm{Si} 2$ & O5 & $109.75(17)$ & $\mathrm{C} 27$ & $\mathrm{C} 28$ & $\mathrm{C} 29$ & $119.0(10)$ \\
\hline O11 & $\mathrm{Si} 2$ & $\mathrm{C} 7$ & $109.73(18)$ & $\mathrm{C} 28$ & $\mathrm{C} 29$ & C30 & $121.9(10)$ \\
\hline $\mathrm{O} 2$ & $\mathrm{Si} 2$ & C7 & 108.31(19) & C25 & C30 & C29 & $118.2(11)$ \\
\hline $\mathrm{O} 5$ & $\mathrm{Si} 2$ & $\mathrm{C} 7$ & $109.8(2)$ & C36A & C31A & C32 & $115.4(10)$ \\
\hline $\mathrm{O} 5$ & $\mathrm{Si} 3$ & O4 & 108.93(19) & C36A & C31A & Si6 & $124.3(9)$ \\
\hline $\mathrm{O} 5$ & $\mathrm{Si} 3$ & O8 & 109.6(2) & C32 & C31A & $\mathrm{Si} 6$ & $119.5(7)$ \\
\hline $\mathrm{O} 4$ & $\mathrm{Si} 3$ & O8 & $110.5(2)$ & $\mathrm{C} 35$ & C34 & C33 & 120.0 \\
\hline $\mathrm{O} 5$ & $\mathrm{Si} 3$ & $\mathrm{C} 13$ & $109.8(4)$ & $\mathrm{C} 36$ & $\mathrm{C} 35$ & C34 & 120.0 \\
\hline $\mathrm{O} 4$ & $\mathrm{Si} 3$ & $\mathrm{C} 13$ & $108.9(3)$ & C35 & $\mathrm{C} 36$ & C31 & 120.0 \\
\hline $\mathrm{O} 8$ & $\mathrm{Si} 3$ & C13 & 109.0(3) & C36 & C31 & C32 & 120.0 \\
\hline O6 & $\mathrm{Si} 4$ & $\mathrm{O} 3$ & $108.50(18)$ & $\mathrm{C} 36$ & C31 & $\mathrm{Si} 6$ & $120.4(3)$ \\
\hline O6 & $\mathrm{Si} 4$ & O4 & $109.5(2)$ & C32 & C31 & $\mathrm{Si} 6$ & $118.7(4)$ \\
\hline $\mathrm{O} 3$ & $\mathrm{Si} 4$ & $\mathrm{O} 4$ & $109.21(17)$ & C31A & C32 & C33 & $121.9(6)$ \\
\hline O6 & $\mathrm{Si} 4$ & C19 & $110.8(2)$ & C33 & C32 & C31 & 120.0 \\
\hline $\mathrm{O} 3$ & $\mathrm{Si} 4$ & C19 & $110.41(19)$ & C32 & C33 & C34 & 120.0 \\
\hline $\mathrm{O} 4$ & $\mathrm{Si} 4$ & C19 & $108.4(2)$ & C32 & C33 & C34A & $115.0(7)$ \\
\hline O9 & $\mathrm{Si} 5$ & $\mathrm{O} 7$ & $112.26(18)$ & C35A & C34A & C33 & $118.3(12)$ \\
\hline O9 & $\mathrm{Si} 5$ & O6 & $112.57(17)$ & C34A & C35A & C36A & $119.7(13)$ \\
\hline
\end{tabular}




\begin{tabular}{|c|c|c|c|c|c|c|c|}
\hline Atom & Atom & Atom & Angle $/^{\circ}$ & Atom & Atom & Atom & Angle $/^{\circ}$ \\
\hline $\mathrm{O} 7$ & $\mathrm{Si} 5$ & O6 & $105.3(2)$ & C34A & C35A & $\mathrm{C} 35 \mathrm{~A}^{1}$ & $148(2)$ \\
\hline O9 & $\mathrm{Si} 5$ & $\mathrm{C} 25$ & $112.5(4)$ & C36A & $\mathrm{C} 35 \mathrm{~A}$ & $\mathrm{C} 35 \mathrm{~A}^{1}$ & $78.1(13)$ \\
\hline $\mathrm{O} 7$ & $\mathrm{Si} 5$ & $\mathrm{C} 25$ & $106.4(3)$ & $\mathrm{C} 31 \mathrm{~A}$ & C36A & $\mathrm{C} 35 \mathrm{~A}$ & $120.9(13)$ \\
\hline O6 & Si5 & $\mathrm{C} 25$ & $107.3(3)$ & $\mathrm{C} 42$ & $\mathrm{C} 37$ & C38 & $117.4(5)$ \\
\hline $\mathrm{O} 10$ & Si6 & $\mathrm{O} 7$ & $111.9(2)$ & $\mathrm{C} 42$ & $\mathrm{C} 37$ & $\mathrm{Si} 7$ & $120.3(4)$ \\
\hline $\mathrm{O} 10$ & $\mathrm{Si} 6$ & O8 & $108.8(2)$ & $\mathrm{C} 38$ & $\mathrm{C} 37$ & $\mathrm{Si} 7$ & $122.3(4)$ \\
\hline $\mathrm{O} 7$ & $\mathrm{Si} 6$ & $\mathrm{O} 8$ & $109.1(2)$ & C37 & $\mathrm{C} 38$ & C39 & $120.3(7)$ \\
\hline $\mathrm{O} 10$ & $\mathrm{Si} 6$ & C31 & $112.4(2)$ & $\mathrm{C} 40$ & C39 & C38 & $120.7(8)$ \\
\hline $\mathrm{O} 7$ & Si6 & C31 & $116.0(3)$ & $\mathrm{C} 41$ & $\mathrm{C} 40$ & C39 & $119.8(6)$ \\
\hline $\mathrm{O} 8$ & $\mathrm{Si} 6$ & C31 & $97.5(3)$ & $\mathrm{C} 40$ & $\mathrm{C} 41$ & $\mathrm{C} 42$ & $121.2(7)$ \\
\hline $\mathrm{O} 10$ & $\mathrm{Si} 6$ & C31A & $105.8(4)$ & C37 & $\mathrm{C} 42$ & C41 & $120.5(6)$ \\
\hline $\mathrm{O} 7$ & $\mathrm{Si} 6$ & C31A & $100.8(4)$ & $\mathrm{C} 48$ & $\mathrm{C} 43$ & C44 & $116.7(5)$ \\
\hline O8 & $\mathrm{Si} 6$ & C31A & $120.3(5)$ & $\mathrm{C} 48$ & $\mathrm{C} 43$ & Si8 & $122.8(4)$ \\
\hline $\mathrm{O} 12$ & $\mathrm{Si} 7$ & O10 & $110.63(17)$ & $\mathrm{C} 44$ & $\mathrm{C} 43$ & $\mathrm{Si} 8$ & $120.5(4)$ \\
\hline $\mathrm{O} 12$ & Si7 & O11 & $112.92(15)$ & $\mathrm{C} 43$ & $\mathrm{C} 44$ & $\mathrm{C} 45$ & $122.2(7)$ \\
\hline $\mathrm{O} 10$ & Si7 & O11 & $107.74(17)$ & $\mathrm{C} 46$ & $\mathrm{C} 45$ & C44 & $120.3(7)$ \\
\hline $\mathrm{O} 12$ & Si7 & C37 & $114.07(19)$ & $\mathrm{C} 45$ & $\mathrm{C} 46$ & $\mathrm{C} 47$ & $119.0(5)$ \\
\hline $\mathrm{O} 10$ & $\mathrm{Si} 7$ & C37 & $106.3(2)$ & $\mathrm{C} 46$ & C47 & $\mathrm{C} 48$ & $120.4(7)$ \\
\hline O11 & $\mathrm{Si} 7$ & C37 & $104.7(2)$ & $\mathrm{C} 43$ & $\mathrm{C} 48$ & $\mathrm{C} 47$ & $121.2(6)$ \\
\hline $\mathrm{O} 13$ & Si8 & O17 & $111.88(18)$ & $\mathrm{C} 54$ & C49 & C50 & $116.5(5)$ \\
\hline O13 & $\mathrm{Si} 8$ & $\mathrm{O} 14$ & $112.39(18)$ & C54 & C49 & Si9 & $122.9(4)$ \\
\hline O17 & Si8 & $\mathrm{O} 14$ & $107.03(18)$ & C50 & C49 & Si9 & $120.6(5)$ \\
\hline $\mathrm{O} 13$ & $\mathrm{Si} 8$ & $\mathrm{C} 43$ & $114.25(17)$ & C51 & C50 & C49 & $120.2(6)$ \\
\hline O17 & $\mathrm{Si} 8$ & $\mathrm{C} 43$ & $105.4(2)$ & C52 & C51 & $\mathrm{C} 50$ & $121.8(6)$ \\
\hline O14 & $\mathrm{Si} 8$ & $\mathrm{C} 43$ & $105.3(2)$ & C51 & C52 & C53 & $118.6(7)$ \\
\hline $\mathrm{O} 15$ & Si9 & $\mathrm{O} 14$ & $108.60(17)$ & C52 & C53 & C54 & $121.2(7)$ \\
\hline $\mathrm{O} 15$ & Si9 & O19 & $109.97(16)$ & C53 & C54 & C49 & $121.8(6)$ \\
\hline O14 & Si9 & O19 & $109.45(19)$ & C60 & C55 & C56 & $117.3(5)$ \\
\hline $\mathrm{O} 15$ & $\mathrm{Si} 9$ & C49 & $111.0(2)$ & C60 & C55 & Sil0 & $122.3(4)$ \\
\hline O14 & $\mathrm{Si} 9$ & C49 & $110.8(2)$ & C56 & C55 & Sil0 & $120.4(4)$ \\
\hline O19 & Si9 & C49 & $107.0(2)$ & C57 & C56 & C55 & $120.7(6)$ \\
\hline O16 & Sil0 & $\mathrm{O} 15$ & $108.72(16)$ & C58 & C57 & C56 & $120.5(7)$ \\
\hline O16 & Sil0 & $\mathrm{O} 21$ & 111.51(16) & C59 & C58 & C57 & $119.8(6)$ \\
\hline 015 & $\mathrm{Si} 10$ & $\mathrm{O} 21$ & $107.46(16)$ & C58 & C59 & C60 & $119.6(6)$ \\
\hline O16 & Si10 & C55 & $113.98(18)$ & C59 & C60 & C55 & $122.0(5)$ \\
\hline O15 & Si10 & C55 & $107.7(2)$ & C66 & C61 & C62 & $117.4(4)$ \\
\hline $\mathrm{O} 21$ & Sil0 & C55 & $107.25(19)$ & C66 & C61 & Sil1 & $121.4(4)$ \\
\hline $\mathrm{O} 21$ & Si11 & $\mathrm{O} 22$ & $108.89(16)$ & C62 & C61 & Sil1 & $121.2(4)$ \\
\hline $\mathrm{O} 21$ & Si11 & $\mathrm{O} 20$ & 109.93(17) & C61 & C62 & C63 & $121.6(5)$ \\
\hline $\mathrm{O} 22$ & Si11 & $\mathrm{O} 20$ & $110.24(17)$ & C64 & C63 & C62 & $119.5(6)$ \\
\hline $\mathrm{O} 21$ & Si11 & C61 & $109.50(19)$ & C63 & C64 & C65 & $119.6(5)$ \\
\hline
\end{tabular}




\begin{tabular}{|c|c|c|c|c|c|c|c|}
\hline Atom & Atom & Atom & Angle $/^{\circ}$ & Atom & Atom & Atom & Angle ${ }^{\circ}$ \\
\hline $\mathrm{O} 22$ & Si11 & C61 & $107.9(2)$ & C64 & C65 & C66 & $120.3(6)$ \\
\hline $\mathrm{O} 20$ & Si11 & C61 & $110.34(18)$ & C61 & C66 & C65 & $121.4(6)$ \\
\hline O19 & Si12 & $\mathrm{O} 18$ & $110.42(18)$ & C68A & C67A & $\mathrm{C} 72$ & $113.8(6)$ \\
\hline O19 & Si12 & $\mathrm{O} 20$ & $108.33(17)$ & C68A & C67A & Si12 & $126.9(6)$ \\
\hline $\mathrm{O} 18$ & Si12 & $\mathrm{O} 20$ & 109.3(2) & $\mathrm{C} 72$ & C67A & Sil2 & $118.0(5)$ \\
\hline O19 & Si12 & C67A & $109.0(3)$ & C67A & C68A & C69A & $123.6(8)$ \\
\hline $\mathrm{O} 18$ & Si12 & C67A & $109.0(3)$ & C70A & C69A & C68A & $122.5(9)$ \\
\hline $\mathrm{O} 20$ & Si12 & C67A & $110.7(2)$ & C69A & C70A & C71 & $121.3(8)$ \\
\hline $\mathrm{O} 17$ & Si13 & $\mathrm{O} 23$ & $109.00(17)$ & $\mathrm{C} 70 \mathrm{~A}$ & C71 & $\mathrm{C} 72$ & $117.5(9)$ \\
\hline $\mathrm{O} 17$ & Si13 & O18 & $109.4(2)$ & C71 & $\mathrm{C} 72$ & C67A & $119.9(8)$ \\
\hline $\mathrm{O} 23$ & Si13 & O18 & $109.34(18)$ & C74 & $\mathrm{C} 73$ & C78 & $120.3(9)$ \\
\hline $\mathrm{O} 17$ & Si13 & C73 & $110.9(2)$ & C78A & C73 & C74A & $108.4(13)$ \\
\hline $\mathrm{O} 23$ & Si13 & C73 & $112.6(3)$ & C74 & C73 & Si13 & $127.3(8)$ \\
\hline O18 & Si13 & C73 & $105.5(2)$ & C78A & C73 & Si13 & $133.9(11)$ \\
\hline $\mathrm{O} 24$ & Si14 & $\mathrm{O} 23$ & $112.41(16)$ & C78 & C73 & Si13 & $112.2(7)$ \\
\hline $\mathrm{O} 24$ & Si14 & $\mathrm{O} 22$ & $106.43(17)$ & C74A & C73 & Sil3 & $117.5(8)$ \\
\hline $\mathrm{O} 23$ & Si14 & $\mathrm{O} 22$ & 107.71(18) & C73 & C74 & $\mathrm{C} 75$ & $123.0(11)$ \\
\hline $\mathrm{O} 24$ & Si14 & C79 & $116.2(2)$ & $\mathrm{C} 75 \mathrm{~A}$ & C74A & C73 & $121.8(15)$ \\
\hline $\mathrm{O} 23$ & Si14 & C79 & $106.1(2)$ & C76 & C75 & C74 & $118.0(14)$ \\
\hline $\mathrm{O} 22$ & Si14 & C79 & $107.6(2)$ & C74A & C75A & C76A & $120(2)$ \\
\hline Sil & $\mathrm{O} 1$ & $\mathrm{Mn} 2$ & $148.14(16)$ & $\mathrm{C} 75$ & C76 & C77 & $121.4(12)$ \\
\hline Sil & $\mathrm{O} 1$ & Mn3 & $110.69(14)$ & $\mathrm{C} 75 \mathrm{~A}$ & C76A & C77A & $120.8(17)$ \\
\hline $\mathrm{Mn} 2$ & $\mathrm{O} 1$ & Mn3 & $100.97(11)$ & C78 & C77 & C76 & $118.2(11)$ \\
\hline $\mathrm{Si} 2$ & $\mathrm{O} 2$ & Si1 & $137.7(2)$ & C78A & C77A & C76A & $116.3(18)$ \\
\hline $\mathrm{Si} 4$ & $\mathrm{O} 3$ & Sil & $152.7(2)$ & C77 & C78 & C73 & $119.1(11)$ \\
\hline $\mathrm{Si} 3$ & $\mathrm{O} 4$ & $\mathrm{Si} 4$ & $142.6(3)$ & C73 & C78A & C77A & $132(2)$ \\
\hline $\mathrm{Si} 3$ & O5 & $\mathrm{Si} 2$ & $146.3(3)$ & $\mathrm{C} 80$ & C79 & C84 & $118.6(5)$ \\
\hline $\mathrm{Si} 4$ & O6 & $\mathrm{Si} 5$ & $138.9(2)$ & $\mathrm{C} 80$ & C79 & Sil4 & $124.1(4)$ \\
\hline $\mathrm{Si} 6$ & $\mathrm{O} 7$ & $\mathrm{Si} 5$ & $141.9(2)$ & $\mathrm{C} 84$ & C79 & Sil4 & $117.3(5)$ \\
\hline $\mathrm{Si} 6$ & $\mathrm{O} 8$ & $\mathrm{Si} 3$ & $147.9(2)$ & C79 & $\mathrm{C} 80$ & C81 & $119.7(7)$ \\
\hline $\mathrm{Si} 5$ & O9 & Mn3 & $138.7(2)$ & $\mathrm{C} 80$ & $\mathrm{C} 81$ & C82 & $119.4(8)$ \\
\hline $\mathrm{Si} 6$ & O10 & $\mathrm{Si} 7$ & $159.5(2)$ & $\mathrm{C} 83$ & $\mathrm{C} 82$ & C81 & $121.2(8)$ \\
\hline $\mathrm{Si} 2$ & O11 & $\mathrm{Si} 7$ & $156.4(2)$ & $\mathrm{C} 82$ & $\mathrm{C} 83$ & C84 & 119.1(8) \\
\hline $\mathrm{Si} 7$ & O12 & Mn1 & $137.48(17)$ & $\mathrm{C} 83$ & $\mathrm{C} 84$ & C79 & $121.8(7)$ \\
\hline Si7 & $\mathrm{O} 12$ & Mn3 & $127.24(16)$ & N1 & $\mathrm{C} 85$ & C86 & $122.8(9)$ \\
\hline Mn1 & $\mathrm{O} 12$ & Mn3 & $94.83(11)$ & C87 & $\mathrm{C} 86$ & $\mathrm{C} 85$ & $120.4(9)$ \\
\hline $\mathrm{Si} 8$ & O13 & $\mathrm{Mn} 2$ & $154.15(19)$ & $\mathrm{C} 86$ & C87 & C88 & $118.2(7)$ \\
\hline $\mathrm{Si} 9$ & O14 & Si8 & $138.80(19)$ & $\mathrm{C} 87$ & $\mathrm{C} 88$ & C89 & $119.3(8)$ \\
\hline $\mathrm{Si} 9$ & $\mathrm{O} 15$ & Si10 & $148.2(2)$ & N1 & C89 & C88 & $122.9(6)$ \\
\hline Si10 & O16 & $\mathrm{Mn} 2$ & $120.96(14)$ & $\mathrm{N} 2$ & $\mathrm{C} 90$ & C91 & $121.5(6)$ \\
\hline Sil0 & O16 & Mn1 & $128.23(15)$ & C92 & C91 & C90 & $119.8(7)$ \\
\hline
\end{tabular}




\begin{tabular}{llllllllr} 
Atom & Atom & Atom & \multicolumn{1}{c}{ Angle $^{\circ}$} & Atom & Atom & Atom & Angle ${ }^{\circ}$ \\
\hline Mn2 & O16 & Mn1 & $102.19(12)$ & C91 & C92 & C93 & $119.8(7)$ \\
Si13 & O17 & Si8 & $136.0(2)$ & C92 & C93 & C94 & $117.5(7)$ \\
Si12 & O18 & Si13 & $145.3(2)$ & N2 & C94 & C93 & $123.6(7)$ \\
Si12 & O19 & Si9 & $149.4(2)$ & N3 & C95 & C96 & $123.2(7)$ \\
Si12 & O20 & Si11 & $141.0(2)$ & C95 & C96 & C97 & $119.2(7)$ \\
Si11 & O21 & Si10 & $142.7(2)$ & C98 & C97 & C96 & $119.0(6)$ \\
Si11 & O22 & Si14 & $151.0(2)$ & C97 & C98 & C99 & $118.4(6)$ \\
Si13 & O23 & Si14 & $164.5(2)$ & N3 & C99 & C98 & $123.4(6)$ \\
Si14 & O24 & Mn3 & $141.39(18)$ & N4 & C100 & C101 & $120.9(6)$ \\
Si14 & O24 & Mn1 & $112.83(16)$ & C102 & C101 & C100 & $120.1(6)$ \\
Mn3 & O24 & Mn1 & $97.58(12)$ & C103 & C102 & C101 & $119.3(6)$ \\
C89 & N1 & C85 & $116.2(6)$ & C102 & C103 & C104 & $118.9(6)$ \\
C89 & N1 & Mn1 & $118.4(3)$ & N4 & C104 & C103 & $122.4(5)$ \\
C85 & N1 & Mn1 & $125.3(5)$ & C105 & N5 & C109 & $115.8(10)$ \\
C90 & N2 & C94 & $117.8(5)$ & N5 & C105 & C106 & $118.5(11)$ \\
C90 & N2 & Mn1 & $116.8(3)$ & C107 & C106 & C105 & $124.1(14)$ \\
C94 & N2 & Mn1 & $125.5(4)$ & C108 & C107 & C106 & $111.8(14)$ \\
C99 & N3 & C95 & $116.8(5)$ & C107 & C108 & C109 & $126.2(16)$ \\
C99 & N3 & Mn2 & $123.3(4)$ & N5 & C109 & C108 & $118.4(12)$ \\
C95 & N3 & Mn2 & $119.9(4)$ & C114 & N6 & C110 & $116.3(7)$ \\
C104 & N4 & C100 & $118.4(4)$ & N6 & C110 & C111 & $122.8(8)$ \\
C104 & N4 & Mn3 & $120.3(3)$ & C112 & C111 & C110 & $118.1(8)$ \\
C100 & N4 & Mn3 & $121.1(3)$ & C113 & C112 & C111 & $119.2(8)$ \\
C6 & C1 & C2 & $117.1(4)$ & C114 & C113 & C112 & $117.6(8)$ \\
C6 & C1 & Si1 & $119.6(3)$ & N6 & C114 & C113 & $126.0(8)$ \\
\hline
\end{tabular}

${ }^{1}-\mathrm{X}, 1-\mathrm{Y}, 2-\mathrm{Z}$

\section{Experimental}

A single crystal of $\mathrm{C}_{114} \mathrm{H}_{100} \mathrm{Mn}_{3} \mathrm{~N}_{6} \mathrm{O}_{24} \mathrm{Si}_{14}$ was selected and mounted on a diffractometer. The crystal was kept at 100(2) K during data collection. The structure was solved with the ShelXS structure solution program using Structure Expansion and refined with the $\mathrm{XL}^{2}$ refinement package using Least Squares minimization, and $\mathrm{Olex} 2^{4}$ as a GUI. 


\section{Crystal structure determination}

Crystal Data for $\mathrm{C}_{114} \mathrm{H}_{100} \mathrm{Mn}_{3} \mathrm{~N}_{6} \mathrm{O}_{24} \mathrm{Si}_{14}(M=2496.07 \mathrm{~g} / \mathrm{mol})$ : triclinic, space group P-1 (no. 2), $a=14.987(3) \AA, b=16.351(4) \AA, c=29.571(6) \AA, \alpha=76.487(4)^{\circ}, \beta=82.781(4)^{\circ}, \gamma=$ $72.809(4)^{\circ}, V=6718(2) \AA^{3}, Z=2, T=100(2) \mathrm{K}, \mu(\mathrm{MoK} \alpha)=0.467 \mathrm{~mm}^{-1}$, Dcalc $=1.234 \mathrm{~g} / \mathrm{cm}^{3}$, 103688 reflections measured $\left(2.666^{\circ} \leq 2 \Theta \leq 55.89^{\circ}\right), 31955$ unique $\left(R_{\text {int }}=0.0605, R_{\text {sigma }}=0.0751\right)$ which were used in all calculations. The final $R_{1}$ was $0.0739(\mathrm{I}>2 \sigma(\mathrm{I}))$ and $w R_{2}$ was 0.2292 (all data).

\section{Refinement model description}

Number of restraints - 394

This report has been created with Olex2, compiled on 2018.05.29 svn.r3508 for OlexSys. 


\section{Compound $1 \cdot 3$ THF}

Table S4. Crystal data and structure refinement for $1 \cdot 3$ THF.

\begin{tabular}{|c|c|}
\hline Identification code & $1 \cdot 3 \mathrm{THF}$ \\
\hline Empirical formula & $\mathrm{C}_{116} \mathrm{H}_{114} \mathrm{Mn}_{3} \mathrm{~N}_{4} \mathrm{O}_{27} \mathrm{Si}_{14}$ \\
\hline Formula weight & 2554.19 \\
\hline Temperature/K & 99.93 \\
\hline Crystal system & triclinic \\
\hline Space group & P-1 \\
\hline $\mathrm{a} / \AA$ & $14.932(3)$ \\
\hline $\mathrm{b} / \AA$ & $16.109(3)$ \\
\hline $\mathrm{c} / \AA$ & $29.164(6)$ \\
\hline$\alpha /^{\circ}$ & $75.886(4)$ \\
\hline$\beta /{ }^{\circ}$ & $82.173(4)$ \\
\hline$\gamma /{ }^{\circ}$ & $72.330(4)$ \\
\hline Volume $/ \AA^{3}$ & $6468(2)$ \\
\hline $\mathrm{Z}$ & 2 \\
\hline$\rho_{\text {calc }} \mathrm{g} / \mathrm{cm}^{3}$ & 1.312 \\
\hline$\mu / \mathrm{mm}^{-1}$ & 0.487 \\
\hline $\mathrm{F}(000)$ & 2650.0 \\
\hline Crystal size $/ \mathrm{mm}^{3}$ & $0.46 \times 0.18 \times 0.17$ \\
\hline Radiation & $\operatorname{MoK} \alpha(\lambda=0.71073)$ \\
\hline $2 \Theta$ range for data collection $/{ }^{\circ}$ & 2.716 to 52.992 \\
\hline Index ranges & $-15 \leq \mathrm{h} \leq 18,-20 \leq \mathrm{k} \leq 20,-36 \leq 1 \leq 36$ \\
\hline Reflections collected & 101139 \\
\hline Independent reflections & $26323\left[\mathrm{R}_{\mathrm{int}}=0.0563, \mathrm{R}_{\text {sigma }}=0.0601\right]$ \\
\hline Data/restraints/parameters & $26323 / 970 / 1571$ \\
\hline Goodness-of-fit on $\mathrm{F}^{2}$ & 1.036 \\
\hline Final $R$ indexes $[\mathrm{I}>=2 \sigma(\mathrm{I})]$ & $\mathrm{R}_{1}=0.0829, \mathrm{wR}_{2}=0.2051$ \\
\hline Final R indexes [all data] & $\mathrm{R}_{1}=0.1205, \mathrm{wR}_{2}=0.2287$ \\
\hline Largest diff. peak/hole / e $\AA^{-3}$ & $1.45 /-1.10$ \\
\hline
\end{tabular}

Table S5 Bond Lengths for $1 \cdot 3$ THF.

\begin{tabular}{llrllr} 
Atom & Atom & Length $/ \AA$ & Atom & Atom & Length/ \\
\hline Mn1 & Mn2 & $3.2220(12)$ & C25 & C30 & 1.3900 \\
Mn1 & O1 & $2.131(3)$ & C30 & C29 & 1.3900 \\
Mn1 & O2 & $1.979(4)$ & C29 & C28 & 1.3900 \\
Mn1 & O3 & $2.311(4)$ & C28 & C27 & 1.3900 \\
Mn1 & O13 & $2.108(4)$ & C26A & C27A & 1.3900 \\
Mn1 & N1 & $2.309(4)$ & C26A & C25A & 1.3900 \\
Mn2 & O1 & $2.051(3)$ & C27A & C28A & 1.3900 \\
Mn2 & O14 & $2.131(3)$ & C28A & C29A & 1.3900
\end{tabular}




\begin{tabular}{|c|c|c|c|c|c|}
\hline Atom & Atom & Length/Å & Atom & Atom & Length/Å \\
\hline $\mathrm{Mn} 2$ & $\mathrm{O} 15$ & $1.973(3)$ & C29A & $\mathrm{C} 30 \mathrm{~A}$ & 1.3900 \\
\hline $\mathrm{Mn} 2$ & $\mathrm{~N} 2$ & $2.240(5)$ & $\mathrm{C} 30 \mathrm{~A}$ & $\mathrm{C} 25 \mathrm{~A}$ & 1.3900 \\
\hline Mn3 & $\mathrm{O} 3$ & $2.031(4)$ & C31 & C32 & 1.3900 \\
\hline $\mathrm{Mn} 3$ & $\mathrm{O} 13$ & $2.221(4)$ & $\mathrm{C} 31$ & $\mathrm{C} 36$ & 1.3900 \\
\hline Mn3 & $\mathrm{O} 14$ & $2.123(3)$ & C32 & C33 & 1.3900 \\
\hline Mn3 & N3 & $2.277(5)$ & $\mathrm{C} 33$ & C34 & 1.3900 \\
\hline Mn3 & N4 & $2.276(5)$ & $\mathrm{C} 34$ & C35 & 1.3900 \\
\hline Sil & $\mathrm{O} 1$ & $1.587(3)$ & $\mathrm{C} 35$ & $\mathrm{C} 36$ & 1.3900 \\
\hline Sil & O9 & $1.646(4)$ & C31A & C32A & 1.3900 \\
\hline Sil & $\mathrm{O} 10$ & $1.633(4)$ & C31A & C36A & 1.3900 \\
\hline Si1 & $\mathrm{C} 1$ & $1.852(5)$ & $\mathrm{C} 32 \mathrm{~A}$ & C33A & 1.3900 \\
\hline $\mathrm{Si} 2$ & $\mathrm{O} 2$ & $1.571(4)$ & C33A & $\mathrm{C} 34 \mathrm{~A}$ & 1.3900 \\
\hline $\mathrm{Si} 2$ & $\mathrm{O} 4$ & $1.649(5)$ & C34A & C35A & 1.3900 \\
\hline $\mathrm{Si} 2$ & O5 & $1.650(4)$ & $\mathrm{C} 35 \mathrm{~A}$ & C36A & 1.3900 \\
\hline $\mathrm{Si} 2$ & $\mathrm{C} 7$ & $1.862(8)$ & C37 & C38 & $1.385(8)$ \\
\hline $\mathrm{Si} 3$ & $\mathrm{O} 3$ & $1.596(4)$ & C37 & $\mathrm{C} 42$ & $1.393(8)$ \\
\hline $\mathrm{Si} 3$ & O6 & $1.621(4)$ & C38 & C39 & $1.384(9)$ \\
\hline $\mathrm{Si} 3$ & O7 & $1.631(4)$ & C39 & $\mathrm{C} 40$ & $1.356(10)$ \\
\hline $\mathrm{Si} 3$ & $\mathrm{C} 13$ & $1.856(7)$ & $\mathrm{C} 40$ & $\mathrm{C} 41$ & $1.384(10)$ \\
\hline $\mathrm{Si} 4$ & O4 & $1.607(4)$ & $\mathrm{C} 41$ & $\mathrm{C} 42$ & $1.393(8)$ \\
\hline $\mathrm{Si} 4$ & O8 & $1.628(5)$ & $\mathrm{C} 43$ & C44 & $1.352(8)$ \\
\hline $\mathrm{Si} 4$ & O9 & $1.617(4)$ & $\mathrm{C} 43$ & $\mathrm{C} 48$ & $1.376(9)$ \\
\hline $\mathrm{Si} 4$ & C19 & $1.861(7)$ & $\mathrm{C} 44$ & $\mathrm{C} 45$ & $1.396(9)$ \\
\hline $\mathrm{Si} 5$ & $\mathrm{O} 8$ & $1.620(5)$ & $\mathrm{C} 45$ & $\mathrm{C} 46$ & $1.330(11)$ \\
\hline $\mathrm{Si} 5$ & O11 & $1.619(4)$ & $\mathrm{C} 46$ & $\mathrm{C} 47$ & $1.384(11)$ \\
\hline $\mathrm{Si} 5$ & $\mathrm{O} 12$ & $1.617(6)$ & $\mathrm{C} 47$ & $\mathrm{C} 48$ & $1.395(9)$ \\
\hline $\mathrm{Si} 5$ & $\mathrm{C} 25$ & $1.873(8)$ & C49 & $\mathrm{C} 50$ & $1.400(9)$ \\
\hline $\mathrm{Si} 5$ & $\mathrm{C} 25 \mathrm{~A}$ & $1.852(8)$ & C49 & C54 & $1.386(10)$ \\
\hline $\mathrm{Si} 6$ & $\mathrm{O} 5$ & $1.604(5)$ & $\mathrm{C} 50$ & C51 & $1.368(10)$ \\
\hline $\mathrm{Si} 6$ & O6 & $1.607(4)$ & C51 & C52 & $1.348(14)$ \\
\hline $\mathrm{Si} 6$ & $\mathrm{O} 12$ & $1.620(6)$ & C52 & C53 & $1.399(14)$ \\
\hline $\mathrm{Si} 6$ & C31 & $1.870(6)$ & C53 & C54 & $1.391(10)$ \\
\hline $\mathrm{Si} 6$ & C31A & $1.878(11)$ & C55 & C56 & $1.377(8)$ \\
\hline $\mathrm{Si} 7$ & $\mathrm{O} 7$ & $1.601(4)$ & C55 & C60 & $1.359(8)$ \\
\hline Si7 & $\mathrm{O} 10$ & $1.622(4)$ & C56 & C57 & $1.380(8)$ \\
\hline Si7 & O11 & $1.620(4)$ & C57 & C58 & $1.351(10)$ \\
\hline Si7 & C37 & $1.846(5)$ & C58 & C59 & $1.370(11)$ \\
\hline $\mathrm{Si} 8$ & $\mathrm{O} 15$ & $1.572(3)$ & C59 & C60 & $1.376(11)$ \\
\hline $\mathrm{Si} 8$ & $\mathrm{O} 20$ & $1.650(4)$ & C61 & C66 & 1.3900 \\
\hline $\mathrm{Si} 8$ & $\mathrm{O} 24$ & $1.648(4)$ & C61 & C62 & 1.3900 \\
\hline Si8 & $\mathrm{C} 43$ & $1.863(5)$ & C66 & C65 & 1.3900 \\
\hline
\end{tabular}




\begin{tabular}{|c|c|c|c|c|c|}
\hline Atom & Atom & Length/Å & Atom & Atom & Length/Å \\
\hline Si9 & $\mathrm{O} 13$ & $1.601(4)$ & C65 & C64 & 1.3900 \\
\hline $\mathrm{Si} 9$ & O16 & $1.645(4)$ & C64 & C63 & 1.3900 \\
\hline $\mathrm{Si} 9$ & $\mathrm{O} 17$ & $1.621(4)$ & C63 & C62 & 1.3900 \\
\hline $\mathrm{Si} 9$ & C49 & $1.855(6)$ & C61A & C66A & 1.3900 \\
\hline Sil0 & $\mathrm{O} 14$ & $1.596(4)$ & C61A & C62A & 1.3900 \\
\hline Sil0 & $\mathrm{O} 18$ & $1.636(4)$ & C66A & C65A & 1.3900 \\
\hline Sil0 & O19 & $1.643(4)$ & C65A & C64A & 1.3900 \\
\hline Sil0 & C55 & $1.856(6)$ & C64A & C63A & 1.3900 \\
\hline Sil1 & $\mathrm{O} 21$ & $1.626(4)$ & C63A & C62A & 1.3900 \\
\hline Sil1 & $\mathrm{O} 22$ & $1.605(4)$ & C67 & C68 & $1.402(9)$ \\
\hline Sil1 & $\mathrm{O} 23$ & $1.615(4)$ & C67 & C72 & $1.367(10)$ \\
\hline Sil1 & C61 & $1.868(4)$ & C68 & C69 & $1.413(11)$ \\
\hline Sil1 & C61A & $1.805(12)$ & C69 & $\mathrm{C} 70$ & $1.341(14)$ \\
\hline Si12 & O17 & $1.606(4)$ & $\mathrm{C} 70$ & C71 & $1.357(13)$ \\
\hline Si12 & $\mathrm{O} 23$ & $1.623(4)$ & C71 & C72 & $1.420(10)$ \\
\hline Si12 & $\mathrm{O} 24$ & $1.610(4)$ & C73 & C74 & $1.376(10)$ \\
\hline Si12 & C67 & $1.852(6)$ & C73 & C78 & $1.398(8)$ \\
\hline Si13 & O18 & $1.615(4)$ & C74 & C75 & $1.384(11)$ \\
\hline Si13 & $\mathrm{O} 20$ & $1.617(4)$ & C75 & C76 & $1.389(12)$ \\
\hline Si13 & $\mathrm{O} 22$ & $1.625(4)$ & C76 & C77 & $1.353(11)$ \\
\hline Si13 & C73 & $1.844(6)$ & C77 & C78 & $1.401(10)$ \\
\hline Si14 & O16 & $1.616(4)$ & C79 & $\mathrm{C} 80$ & $1.336(10)$ \\
\hline Sil4 & O19 & $1.616(4)$ & C79 & C84 & $1.377(9)$ \\
\hline Si14 & $\mathrm{O} 21$ & $1.621(4)$ & $\mathrm{C} 80$ & C81 & $1.408(11)$ \\
\hline Si14 & C79 & $1.848(5)$ & C81 & $\mathrm{C} 82$ & $1.335(12)$ \\
\hline N1 & $\mathrm{C} 85$ & $1.329(7)$ & $\mathrm{C} 82$ & C83 & $1.304(12)$ \\
\hline N1 & C89 & $1.346(7)$ & $\mathrm{C} 83$ & C84 & $1.381(9)$ \\
\hline N2 & C90 & $1.339(8)$ & $\mathrm{C} 85$ & $\mathrm{C} 86$ & $1.369(8)$ \\
\hline N2 & C94 & $1.339(7)$ & C86 & C87 & $1.386(11)$ \\
\hline N3 & C95 & $1.231(10)$ & C87 & C88 & $1.362(11)$ \\
\hline N3 & C99 & $1.361(11)$ & C88 & C89 & $1.369(9)$ \\
\hline N4 & $\mathrm{C} 100$ & $1.333(8)$ & $\mathrm{C} 90$ & C91 & $1.381(9)$ \\
\hline N4 & $\mathrm{C} 104$ & $1.341(8)$ & C91 & C92 & $1.374(11)$ \\
\hline $\mathrm{C} 1$ & $\mathrm{C} 2$ & $1.394(8)$ & C92 & C93 & $1.365(11)$ \\
\hline $\mathrm{C} 1$ & C6 & $1.390(8)$ & C93 & C94 & $1.374(9)$ \\
\hline $\mathrm{C} 2$ & $\mathrm{C} 3$ & $1.384(8)$ & C95 & C96 & $1.444(12)$ \\
\hline C3 & $\mathrm{C} 4$ & $1.369(9)$ & C96 & C97 & $1.312(13)$ \\
\hline $\mathrm{C} 4$ & C5 & $1.377(10)$ & C97 & C98 & $1.358(14)$ \\
\hline $\mathrm{C} 5$ & C6 & $1.395(9)$ & C98 & C99 & $1.378(13)$ \\
\hline $\mathrm{C} 7$ & $\mathrm{C} 8$ & $1.375(15)$ & $\mathrm{C} 100$ & C101 & $1.389(9)$ \\
\hline $\mathrm{C} 7$ & $\mathrm{C} 12$ & $1.363(14)$ & C101 & C102 & $1.359(13)$ \\
\hline
\end{tabular}




\begin{tabular}{|c|c|c|c|c|c|}
\hline Atom & Atom & Length/Å & Atom & Atom & Length/Å \\
\hline $\mathrm{C} 8$ & C9 & $1.396(11)$ & C102 & C103 & $1.356(14)$ \\
\hline C9 & $\mathrm{C} 10$ & $1.296(15)$ & $\mathrm{C} 103$ & C104 & $1.361(11)$ \\
\hline $\mathrm{C} 10$ & $\mathrm{C} 11$ & $1.302(16)$ & $\mathrm{O} 25$ & $\mathrm{C} 105$ & $1.553(17)$ \\
\hline C11 & $\mathrm{C} 12$ & $1.487(13)$ & $\mathrm{O} 25$ & C108 & $1.460(19)$ \\
\hline C13 & $\mathrm{C} 14$ & $1.384(12)$ & $\mathrm{C} 105$ & C106 & $1.473(9)$ \\
\hline C13 & $\mathrm{C} 18$ & $1.374(13)$ & $\mathrm{C} 106$ & C107 & $1.482(9)$ \\
\hline C14 & $\mathrm{C} 15$ & $1.388(11)$ & C107 & C108 & $1.44(2)$ \\
\hline $\mathrm{C} 15$ & $\mathrm{C} 16$ & $1.320(15)$ & $\mathrm{O} 26$ & C109 & $1.590(16)$ \\
\hline C16 & $\mathrm{C} 17$ & $1.362(15)$ & $\mathrm{O} 26$ & C112 & $1.594(16)$ \\
\hline $\mathrm{C} 17$ & $\mathrm{C} 18$ & $1.426(12)$ & C109 & C110 & $1.428(18)$ \\
\hline C19 & $\mathrm{C} 20$ & $1.382(10)$ & C110 & C111 & $1.391(18)$ \\
\hline C19 & $\mathrm{C} 24$ & $1.358(10)$ & C111 & C112 & $1.426(18)$ \\
\hline $\mathrm{C} 20$ & $\mathrm{C} 21$ & $1.383(10)$ & $\mathrm{O} 27$ & C113 & $1.480(15)$ \\
\hline $\mathrm{C} 21$ & $\mathrm{C} 22$ & $1.347(14)$ & $\mathrm{O} 27$ & C116 & $1.447(15)$ \\
\hline $\mathrm{C} 22$ & $\mathrm{C} 23$ & $1.345(15)$ & C113 & C114 & $1.628(16)$ \\
\hline $\mathrm{C} 23$ & $\mathrm{C} 24$ & $1.416(13)$ & C114 & C115 & $1.509(16)$ \\
\hline $\mathrm{C} 26$ & $\mathrm{C} 25$ & 1.3900 & C115 & C116 & $1.518(16)$ \\
\hline $\mathrm{C} 26$ & $\mathrm{C} 27$ & 1.3900 & & & \\
\hline
\end{tabular}

Table S6 Bond Angles for 1·3 THF.

\begin{tabular}{|c|c|c|c|c|c|c|c|}
\hline Atom & Atom & Atom & Angle ${ }^{\circ}$ & Atom & Atom & Atom & Angle ${ }^{\circ}$ \\
\hline $\mathrm{O} 1$ & Mn1 & Mn2 & $38.70(9)$ & $\mathrm{C} 2$ & $\mathrm{C} 1$ & Sil & $123.2(4)$ \\
\hline $\mathrm{O} 1$ & Mn1 & $\mathrm{O} 3$ & $84.61(12)$ & C6 & $\mathrm{C} 1$ & Sil & $120.2(4)$ \\
\hline $\mathrm{O} 1$ & Mn1 & N1 & $91.06(14)$ & C6 & $\mathrm{C} 1$ & $\mathrm{C} 2$ & $116.6(5)$ \\
\hline $\mathrm{O} 2$ & Mn1 & $\mathrm{Mn} 2$ & $168.46(12)$ & $\mathrm{C} 3$ & $\mathrm{C} 2$ & $\mathrm{C} 1$ & $122.4(6)$ \\
\hline $\mathrm{O} 2$ & Mn1 & $\mathrm{O} 1$ & $147.09(15)$ & $\mathrm{C} 4$ & C3 & $\mathrm{C} 2$ & $119.6(6)$ \\
\hline $\mathrm{O} 2$ & Mn1 & $\mathrm{O} 3$ & $95.85(14)$ & C3 & $\mathrm{C} 4$ & $\mathrm{C} 5$ & $119.9(6)$ \\
\hline $\mathrm{O} 2$ & Mn1 & O13 & $121.36(16)$ & $\mathrm{C} 4$ & $\mathrm{C} 5$ & C6 & $120.1(6)$ \\
\hline $\mathrm{O} 2$ & Mn1 & N1 & $93.25(16)$ & $\mathrm{C} 1$ & C6 & $\mathrm{C} 5$ & $121.3(6)$ \\
\hline $\mathrm{O} 3$ & Mn1 & $\mathrm{Mn} 2$ & 73.61(9) & $\mathrm{C} 8$ & $\mathrm{C} 7$ & $\mathrm{Si} 2$ & $120.7(8)$ \\
\hline $\mathrm{O} 13$ & Mn1 & $\mathrm{Mn} 2$ & $53.22(10)$ & $\mathrm{C} 12$ & $\mathrm{C} 7$ & $\mathrm{Si} 2$ & $121.3(9)$ \\
\hline $\mathrm{O} 13$ & Mn1 & $\mathrm{O} 1$ & $91.26(13)$ & $\mathrm{C} 12$ & $\mathrm{C} 7$ & $\mathrm{C} 8$ & $117.4(8)$ \\
\hline $\mathrm{O} 13$ & Mn1 & $\mathrm{O} 3$ & $80.45(13)$ & $\mathrm{C} 7$ & $\mathrm{C} 8$ & C9 & $122.7(11)$ \\
\hline $\mathrm{O} 13$ & Mn1 & N1 & $89.61(15)$ & $\mathrm{C} 10$ & C9 & $\mathrm{C} 8$ & $119.7(12)$ \\
\hline N1 & Mn1 & Mn2 & $96.77(11)$ & C9 & $\mathrm{C} 10$ & $\mathrm{C} 11$ & $121.3(10)$ \\
\hline N1 & Mn1 & $\mathrm{O} 3$ & $169.04(15)$ & $\mathrm{C} 10$ & $\mathrm{C} 11$ & $\mathrm{C} 12$ & $121.6(11)$ \\
\hline O1 & Mn2 & Mn1 & $40.52(9)$ & $\mathrm{C} 7$ & $\mathrm{C} 12$ & $\mathrm{C} 11$ & $116.6(11)$ \\
\hline $\mathrm{O} 1$ & $\mathrm{Mn} 2$ & $\mathrm{O} 14$ & $128.30(13)$ & $\mathrm{C} 14$ & $\mathrm{C} 13$ & $\mathrm{Si} 3$ & $121.7(7)$ \\
\hline $\mathrm{O} 1$ & $\operatorname{Mn} 2$ & N2 & $97.77(14)$ & $\mathrm{C} 18$ & $\mathrm{C} 13$ & $\mathrm{Si} 3$ & $120.6(7)$ \\
\hline
\end{tabular}




\begin{tabular}{|c|c|c|c|c|c|c|c|}
\hline Atom & Atom & Atom & Angle $/^{\circ}$ & Atom & Atom & Atom & Angle $/^{\circ}$ \\
\hline $\mathrm{O} 14$ & Mn2 & Mn1 & $98.80(9)$ & $\mathrm{C} 18$ & $\mathrm{C} 13$ & $\mathrm{C} 14$ & $117.6(8)$ \\
\hline $\mathrm{O} 14$ & $\mathrm{Mn} 2$ & N2 & $92.98(15)$ & $\mathrm{C} 13$ & $\mathrm{C} 14$ & $\mathrm{C} 15$ & $121.4(10)$ \\
\hline $\mathrm{O} 15$ & $\mathrm{Mn} 2$ & Mn1 & $111.23(11)$ & $\mathrm{C} 16$ & $\mathrm{C} 15$ & $\mathrm{C} 14$ & $119.9(11)$ \\
\hline $\mathrm{O} 15$ & $\mathrm{Mn} 2$ & $\mathrm{O} 1$ & $102.23(14)$ & $\mathrm{C} 15$ & $\mathrm{C} 16$ & $\mathrm{C} 17$ & $122.3(9)$ \\
\hline $\mathrm{O} 15$ & $\mathrm{Mn} 2$ & $\mathrm{O} 14$ & $126.07(14)$ & $\mathrm{C} 16$ & $\mathrm{C} 17$ & $\mathrm{C} 18$ & $118.2(10)$ \\
\hline $\mathrm{O} 15$ & $\mathrm{Mn} 2$ & N2 & $98.10(16)$ & $\mathrm{C} 13$ & $\mathrm{C} 18$ & $\mathrm{C} 17$ & $120.5(10)$ \\
\hline N2 & $\mathrm{Mn} 2$ & Mn1 & $131.93(12)$ & $\mathrm{C} 20$ & $\mathrm{C} 19$ & $\mathrm{Si} 4$ & $121.3(5)$ \\
\hline $\mathrm{O} 3$ & Mn3 & $\mathrm{O} 13$ & $84.30(14)$ & $\mathrm{C} 24$ & C19 & $\mathrm{Si} 4$ & $121.0(6)$ \\
\hline $\mathrm{O} 3$ & Mn3 & $\mathrm{O} 14$ & $102.70(13)$ & $\mathrm{C} 24$ & C19 & $\mathrm{C} 20$ & $117.5(7)$ \\
\hline $\mathrm{O} 3$ & Mn3 & N3 & $114.46(19)$ & $\mathrm{C} 19$ & $\mathrm{C} 20$ & $\mathrm{C} 21$ & $121.7(8)$ \\
\hline $\mathrm{O} 3$ & Mn3 & N4 & $95.54(17)$ & $\mathrm{C} 22$ & $\mathrm{C} 21$ & $\mathrm{C} 20$ & $120.1(9)$ \\
\hline $\mathrm{O} 13$ & Mn3 & N3 & $160.68(18)$ & $\mathrm{C} 23$ & $\mathrm{C} 22$ & $\mathrm{C} 21$ & $119.9(9)$ \\
\hline $\mathrm{O} 13$ & Mn3 & N4 & $88.51(16)$ & $\mathrm{C} 22$ & $\mathrm{C} 23$ & $\mathrm{C} 24$ & $120.5(9)$ \\
\hline $\mathrm{O} 14$ & Mn3 & $\mathrm{O} 13$ & $81.30(13)$ & C19 & $\mathrm{C} 24$ & $\mathrm{C} 23$ & $120.3(9)$ \\
\hline $\mathrm{O} 14$ & Mn3 & N3 & $98.02(18)$ & $\mathrm{C} 25$ & $\mathrm{C} 26$ & $\mathrm{C} 27$ & 120.0 \\
\hline $\mathrm{O} 14$ & Mn3 & N4 & $158.02(16)$ & $\mathrm{C} 26$ & $\mathrm{C} 25$ & $\mathrm{Si} 5$ & $118.4(7)$ \\
\hline N3 & Mn3 & N4 & $85.2(2)$ & $\mathrm{C} 26$ & $\mathrm{C} 25$ & $\mathrm{C} 30$ & 120.0 \\
\hline $\mathrm{O} 1$ & Sil & O9 & $104.07(19)$ & $\mathrm{C} 30$ & $\mathrm{C} 25$ & $\mathrm{Si} 5$ & $121.5(7)$ \\
\hline $\mathrm{O} 1$ & Sil & $\mathrm{O} 10$ & 113.99(19) & $\mathrm{C} 29$ & $\mathrm{C} 30$ & $\mathrm{C} 25$ & 120.0 \\
\hline $\mathrm{O} 1$ & Sil & $\mathrm{C} 1$ & $115.5(2)$ & $\mathrm{C} 30$ & $\mathrm{C} 29$ & $\mathrm{C} 28$ & 120.0 \\
\hline O9 & Sil & $\mathrm{C} 1$ & $109.8(2)$ & $\mathrm{C} 27$ & $\mathrm{C} 28$ & $\mathrm{C} 29$ & 120.0 \\
\hline $\mathrm{O} 10$ & Sil & O9 & 106.61(19) & $\mathrm{C} 28$ & $\mathrm{C} 27$ & $\mathrm{C} 26$ & 120.0 \\
\hline $\mathrm{O} 10$ & Sil & $\mathrm{C} 1$ & $106.5(2)$ & C27A & C26A & $\mathrm{C} 25 \mathrm{~A}$ & 120.0 \\
\hline $\mathrm{O} 2$ & $\mathrm{Si} 2$ & O4 & $112.1(2)$ & C26A & C27A & C28A & 120.0 \\
\hline $\mathrm{O} 2$ & $\mathrm{Si} 2$ & O5 & $112.7(2)$ & C29A & C28A & C27A & 120.0 \\
\hline $\mathrm{O} 2$ & $\mathrm{Si} 2$ & $\mathrm{C} 7$ & $111.9(4)$ & C28A & C29A & C30A & 120.0 \\
\hline $\mathrm{O} 4$ & $\mathrm{Si} 2$ & O5 & $105.3(3)$ & $\mathrm{C} 25 \mathrm{~A}$ & C30A & C29A & 120.0 \\
\hline $\mathrm{O} 4$ & $\mathrm{Si} 2$ & $\mathrm{C} 7$ & $107.1(4)$ & C26A & $\mathrm{C} 25 \mathrm{~A}$ & $\mathrm{Si} 5$ & $121.6(7)$ \\
\hline $\mathrm{O} 5$ & $\mathrm{Si} 2$ & $\mathrm{C} 7$ & $107.2(4)$ & C30A & $\mathrm{C} 25 \mathrm{~A}$ & $\mathrm{Si} 5$ & $118.2(7)$ \\
\hline $\mathrm{O} 3$ & $\mathrm{Si} 3$ & O6 & $111.3(2)$ & C30A & $\mathrm{C} 25 \mathrm{~A}$ & C26A & 120.0 \\
\hline $\mathrm{O} 3$ & $\mathrm{Si} 3$ & $\mathrm{O} 7$ & 111.93(19) & C32 & C31 & $\mathrm{Si} 6$ & $117.8(6)$ \\
\hline $\mathrm{O} 3$ & $\mathrm{Si} 3$ & $\mathrm{C} 13$ & $112.6(3)$ & C32 & C31 & $\mathrm{C} 36$ & 120.0 \\
\hline O6 & $\mathrm{Si} 3$ & O7 & $107.5(2)$ & C36 & C31 & Si6 & $122.2(6)$ \\
\hline O6 & $\mathrm{Si} 3$ & C13 & $107.4(4)$ & C33 & $\mathrm{C} 32$ & $\mathrm{C} 31$ & 120.0 \\
\hline $\mathrm{O} 7$ & $\mathrm{Si} 3$ & C13 & $105.8(3)$ & C32 & C33 & C34 & 120.0 \\
\hline $\mathrm{O} 4$ & $\mathrm{Si} 4$ & O8 & $109.3(3)$ & C33 & C34 & $\mathrm{C} 35$ & 120.0 \\
\hline $\mathrm{O} 4$ & $\mathrm{Si} 4$ & O9 & $108.7(2)$ & $\mathrm{C} 36$ & $\mathrm{C} 35$ & C34 & 120.0 \\
\hline $\mathrm{O} 4$ & $\mathrm{Si} 4$ & C19 & $109.8(2)$ & $\mathrm{C} 35$ & C36 & C31 & 120.0 \\
\hline O8 & $\mathrm{Si} 4$ & C19 & $109.4(3)$ & C32A & C31A & Si6 & $121.5(9)$ \\
\hline O9 & $\mathrm{Si} 4$ & O8 & $108.9(2)$ & C32A & C31A & C36A & 120.0 \\
\hline O9 & $\mathrm{Si} 4$ & C19 & $110.7(3)$ & C36A & C31A & Si6 & $118.4(9)$ \\
\hline
\end{tabular}




\begin{tabular}{|c|c|c|c|c|c|c|c|}
\hline Atom & Atom & Atom & Angle $/^{\circ}$ & Atom & Atom & Atom & Angle $/^{\circ}$ \\
\hline$\overline{\mathrm{O} 8}$ & Si5 & $\mathrm{C} 25$ & $112.2(4)$ & C33A & C32A & C31A & 120.0 \\
\hline $\mathrm{O} 8$ & $\mathrm{Si} 5$ & $\mathrm{C} 25 \mathrm{~A}$ & $102.2(4)$ & C32A & C33A & C34A & 120.0 \\
\hline $\mathrm{O} 11$ & $\mathrm{Si} 5$ & $\mathrm{O} 8$ & $109.4(2)$ & C35A & C34A & C33A & 120.0 \\
\hline $\mathrm{O} 11$ & $\mathrm{Si} 5$ & $\mathrm{C} 25$ & $107.2(4)$ & C36A & $\mathrm{C} 35 \mathrm{~A}$ & C34A & 120.0 \\
\hline $\mathrm{O} 11$ & $\mathrm{Si} 5$ & $\mathrm{C} 25 \mathrm{~A}$ & $111.1(4)$ & $\mathrm{C} 35 \mathrm{~A}$ & C36A & C31A & 120.0 \\
\hline $\mathrm{O} 12$ & $\mathrm{Si} 5$ & $\mathrm{O} 8$ & $110.3(3)$ & $\mathrm{C} 38$ & C37 & $\mathrm{Si} 7$ & $122.5(4)$ \\
\hline $\mathrm{O} 12$ & $\mathrm{Si} 5$ & O11 & $109.5(3)$ & C38 & C37 & $\mathrm{C} 42$ & $117.9(5)$ \\
\hline $\mathrm{O} 12$ & $\mathrm{Si} 5$ & $\mathrm{C} 25$ & $108.1(4)$ & $\mathrm{C} 42$ & $\mathrm{C} 37$ & $\mathrm{Si7}$ & $119.7(4)$ \\
\hline $\mathrm{O} 12$ & $\mathrm{Si} 5$ & $\mathrm{C} 25 \mathrm{~A}$ & $114.0(5)$ & $\mathrm{C} 39$ & $\mathrm{C} 38$ & $\mathrm{C} 37$ & $121.6(6)$ \\
\hline $\mathrm{O} 5$ & $\mathrm{Si} 6$ & O6 & $110.1(2)$ & $\mathrm{C} 40$ & C39 & C38 & $119.6(6)$ \\
\hline $\mathrm{O} 5$ & $\mathrm{Si} 6$ & $\mathrm{O} 12$ & $110.4(3)$ & C39 & $\mathrm{C} 40$ & C41 & $121.2(6)$ \\
\hline O5 & $\mathrm{Si} 6$ & C31 & $114.3(4)$ & $\mathrm{C} 40$ & $\mathrm{C} 41$ & $\mathrm{C} 42$ & $119.0(6)$ \\
\hline $\mathrm{O} 5$ & $\mathrm{Si} 6$ & C31A & $102.8(5)$ & $\mathrm{C} 41$ & $\mathrm{C} 42$ & C37 & $120.8(6)$ \\
\hline O6 & Si6 & O12 & $109.4(2)$ & $\mathrm{C} 44$ & $\mathrm{C} 43$ & Si8 & $121.6(4)$ \\
\hline O6 & $\mathrm{Si} 6$ & $\mathrm{C} 31$ & $111.0(4)$ & $\mathrm{C} 44$ & $\mathrm{C} 43$ & $\mathrm{C} 48$ & $116.5(5)$ \\
\hline O6 & $\mathrm{Si} 6$ & C31A & $107.3(6)$ & $\mathrm{C} 48$ & $\mathrm{C} 43$ & Si8 & $121.9(4)$ \\
\hline $\mathrm{O} 12$ & $\mathrm{Si} 6$ & C31 & $101.3(4)$ & $\mathrm{C} 43$ & $\mathrm{C} 44$ & $\mathrm{C} 45$ & $122.8(7)$ \\
\hline $\mathrm{O} 12$ & Si6 & C31A & $116.7(6)$ & $\mathrm{C} 46$ & $\mathrm{C} 45$ & $\mathrm{C} 44$ & $120.4(7)$ \\
\hline $\mathrm{O} 7$ & $\mathrm{Si} 7$ & O10 & $110.0(2)$ & $\mathrm{C} 45$ & $\mathrm{C} 46$ & $\mathrm{C} 47$ & $118.8(6)$ \\
\hline $\mathrm{O} 7$ & $\mathrm{Si} 7$ & O11 & $109.3(2)$ & $\mathrm{C} 46$ & $\mathrm{C} 47$ & $\mathrm{C} 48$ & $120.0(7)$ \\
\hline O7 & $\mathrm{Si} 7$ & C37 & $109.2(2)$ & $\mathrm{C} 43$ & $\mathrm{C} 48$ & C47 & $121.4(7)$ \\
\hline $\mathrm{O} 10$ & $\mathrm{Si} 7$ & C37 & $109.1(2)$ & C50 & C49 & $\mathrm{Si} 9$ & $117.6(5)$ \\
\hline O11 & $\mathrm{Si} 7$ & O10 & $109.4(2)$ & C54 & C49 & Si9 & $124.8(5)$ \\
\hline O11 & $\mathrm{Si} 7$ & C37 & $109.8(2)$ & C54 & C49 & $\mathrm{C} 50$ & $117.7(6)$ \\
\hline $\mathrm{O} 15$ & $\mathrm{Si} 8$ & $\mathrm{O} 20$ & $111.4(2)$ & C51 & C50 & C49 & $122.0(8)$ \\
\hline O15 & $\mathrm{Si} 8$ & $\mathrm{O} 24$ & $112.5(2)$ & C52 & C51 & $\mathrm{C} 50$ & $119.3(8)$ \\
\hline $\mathrm{O} 15$ & $\mathrm{Si} 8$ & $\mathrm{C} 43$ & $114.0(2)$ & C51 & C52 & C53 & $121.5(8)$ \\
\hline $\mathrm{O} 20$ & $\mathrm{Si} 8$ & $\mathrm{C} 43$ & $105.6(2)$ & C54 & C53 & C52 & $118.7(9)$ \\
\hline $\mathrm{O} 24$ & $\mathrm{Si} 8$ & $\mathrm{O} 20$ & $107.4(2)$ & C49 & C54 & C53 & $120.8(8)$ \\
\hline $\mathrm{O} 24$ & Si8 & $\mathrm{C} 43$ & $105.3(2)$ & C56 & C55 & Silo & $121.4(4)$ \\
\hline $\mathrm{O} 13$ & Si9 & O16 & $106.19(19)$ & C60 & C55 & Sil0 & $121.7(5)$ \\
\hline O13 & $\mathrm{Si} 9$ & O17 & $112.4(2)$ & C60 & C55 & C56 & $116.9(6)$ \\
\hline O13 & Si9 & C49 & $116.4(3)$ & C55 & C56 & C57 & $122.0(6)$ \\
\hline 016 & $\mathrm{Si} 9$ & C49 & $106.7(2)$ & C58 & C57 & C56 & $120.0(6)$ \\
\hline $\mathrm{O} 17$ & $\mathrm{Si} 9$ & O16 & $107.8(2)$ & C57 & C58 & C59 & $119.0(6)$ \\
\hline $\mathrm{O} 17$ & $\mathrm{Si} 9$ & $\mathrm{C} 49$ & $107.0(2)$ & C58 & C59 & C60 & $120.5(7)$ \\
\hline O14 & Sil0 & $\mathrm{O} 18$ & 109.31(19) & C55 & $\mathrm{C} 60$ & C59 & $121.7(7)$ \\
\hline $\mathrm{O} 14$ & Sil0 & O19 & $111.36(19)$ & C66 & C61 & Si11 & $121.4(3)$ \\
\hline $\mathrm{O} 14$ & Sil0 & C55 & $113.5(2)$ & C66 & C61 & C62 & 120.0 \\
\hline O18 & Sil0 & O19 & $108.04(19)$ & C62 & C61 & Sil1 & $118.6(3)$ \\
\hline O18 & $\mathrm{Si} 10$ & C55 & $108.0(2)$ & C61 & C66 & C65 & 120.0 \\
\hline
\end{tabular}




\begin{tabular}{|c|c|c|c|c|c|c|c|}
\hline Atom & Atom & Atom & Angle $/^{\circ}$ & Atom & Atom & Atom & Angle ${ }^{\circ}$ \\
\hline $\mathrm{O} 19$ & Si10 & C55 & $106.5(2)$ & C64 & C65 & C66 & 120.0 \\
\hline $\mathrm{O} 21$ & Sil1 & C61 & $110.9(2)$ & C63 & C64 & C65 & 120.0 \\
\hline $\mathrm{O} 21$ & Si11 & C61A & $107.5(6)$ & C64 & C63 & C62 & 120.0 \\
\hline $\mathrm{O} 22$ & Si11 & $\mathrm{O} 21$ & $108.0(2)$ & C63 & C62 & C61 & 120.0 \\
\hline $\mathrm{O} 22$ & Si11 & $\mathrm{O} 23$ & $110.7(2)$ & C66A & C61A & Si11 & $118.2(10)$ \\
\hline $\mathrm{O} 22$ & Si11 & C61 & $105.5(2)$ & C66A & C61A & C62A & 120.0 \\
\hline $\mathrm{O} 22$ & Si11 & C61A & $120.2(7)$ & C62A & C61A & Si11 & $121.2(10)$ \\
\hline $\mathrm{O} 23$ & Si11 & $\mathrm{O} 21$ & $108.9(2)$ & C61A & C66A & C65A & 120.0 \\
\hline $\mathrm{O} 23$ & Sil1 & C61 & $112.8(2)$ & C66A & C65A & C64A & 120.0 \\
\hline $\mathrm{O} 23$ & Si11 & C61A & $101.1(7)$ & C65A & C64A & C63A & 120.0 \\
\hline $\mathrm{O} 17$ & Si12 & $\mathrm{O} 23$ & $109.6(2)$ & C62A & C63A & C64A & 120.0 \\
\hline $\mathrm{O} 17$ & Si12 & $\mathrm{O} 24$ & $109.2(2)$ & C63A & C62A & C61A & 120.0 \\
\hline O17 & Si12 & C67 & $111.7(3)$ & C68 & C67 & Si12 & $118.9(6)$ \\
\hline $\mathrm{O} 23$ & Si12 & C67 & $106.1(2)$ & $\mathrm{C} 72$ & C67 & Si12 & $122.7(5)$ \\
\hline $\mathrm{O} 24$ & Si12 & $\mathrm{O} 23$ & $109.1(2)$ & C72 & C67 & C68 & $118.3(6)$ \\
\hline $\mathrm{O} 24$ & Si12 & C67 & $111.1(2)$ & C67 & C68 & C69 & $119.6(8)$ \\
\hline $\mathrm{O} 18$ & Si13 & $\mathrm{O} 20$ & $108.9(2)$ & $\mathrm{C} 70$ & C69 & C68 & $120.5(8)$ \\
\hline $\mathrm{O} 18$ & Si13 & $\mathrm{O} 22$ & $109.38(19)$ & C69 & C70 & C71 & $121.4(8)$ \\
\hline $\mathrm{O} 18$ & Si13 & C73 & $111.8(3)$ & C70 & C71 & $\mathrm{C} 72$ & 119.1(9) \\
\hline $\mathrm{O} 20$ & Si13 & $\mathrm{O} 22$ & $109.2(2)$ & C67 & C72 & C71 & $121.2(7)$ \\
\hline $\mathrm{O} 20$ & Si13 & C73 & $110.3(2)$ & C74 & C73 & Si13 & $122.4(5)$ \\
\hline $\mathrm{O} 22$ & Si13 & C73 & $107.2(2)$ & C74 & C73 & C78 & $118.3(6)$ \\
\hline $\mathrm{O} 16$ & Si14 & $\mathrm{O} 21$ & $109.9(2)$ & $\mathrm{C} 78$ & C73 & Sil3 & $119.2(5)$ \\
\hline 016 & Si14 & C79 & $108.0(3)$ & C73 & C74 & $\mathrm{C} 75$ & $120.6(7)$ \\
\hline O19 & Si14 & O16 & $109.39(19)$ & C74 & C75 & C76 & $121.1(8)$ \\
\hline O19 & Si14 & $\mathrm{O} 21$ & $110.3(2)$ & C77 & C76 & $\mathrm{C} 75$ & $118.7(7)$ \\
\hline O19 & Si14 & C79 & $109.5(2)$ & C76 & C77 & $\mathrm{C} 78$ & $121.2(7)$ \\
\hline $\mathrm{O} 21$ & Si14 & C79 & $109.8(2)$ & C73 & $\mathrm{C} 78$ & C77 & $120.0(7)$ \\
\hline Mn2 & $\mathrm{O} 1$ & Mn1 & $100.78(14)$ & $\mathrm{C} 80$ & C79 & Si14 & $121.5(5)$ \\
\hline Sil & $\mathrm{O} 1$ & Mn1 & $110.68(17)$ & $\mathrm{C} 80$ & C79 & C84 & $117.7(6)$ \\
\hline Sil & $\mathrm{O} 1$ & $\operatorname{Mn} 2$ & $148.5(2)$ & C84 & C79 & Sil4 & $120.6(5)$ \\
\hline $\mathrm{Si} 2$ & $\mathrm{O} 2$ & Mn1 & $138.7(2)$ & C79 & $\mathrm{C} 80$ & C81 & $120.1(8)$ \\
\hline Mn3 & $\mathrm{O} 3$ & Mn1 & $96.53(14)$ & $\mathrm{C} 82$ & C81 & $\mathrm{C} 80$ & $121.5(9)$ \\
\hline $\mathrm{Si} 3$ & $\mathrm{O} 3$ & Mn1 & $126.6(2)$ & $\mathrm{C} 83$ & $\mathrm{C} 82$ & C81 & $117.7(8)$ \\
\hline $\mathrm{Si} 3$ & $\mathrm{O} 3$ & Mn3 & $136.8(2)$ & $\mathrm{C} 82$ & C83 & C84 & $123.3(8)$ \\
\hline $\mathrm{Si} 4$ & O4 & $\mathrm{Si} 2$ & $141.1(3)$ & C79 & C84 & C83 & $119.5(7)$ \\
\hline $\mathrm{Si} 6$ & O5 & $\mathrm{Si} 2$ & $138.6(3)$ & N1 & $\mathrm{C} 85$ & C86 & $123.9(6)$ \\
\hline $\mathrm{Si} 6$ & O6 & $\mathrm{Si} 3$ & $161.2(3)$ & $\mathrm{C} 85$ & C86 & C87 & $119.9(7)$ \\
\hline Si7 & $\mathrm{O} 7$ & $\mathrm{Si} 3$ & $156.6(3)$ & C88 & C87 & C86 & $115.6(7)$ \\
\hline $\mathrm{Si} 5$ & $\mathrm{O} 8$ & $\mathrm{Si} 4$ & $141.1(3)$ & C87 & C88 & C89 & $121.8(7)$ \\
\hline $\mathrm{Si} 4$ & O9 & Sil & $152.0(3)$ & N1 & C89 & C88 & $122.3(6)$ \\
\hline
\end{tabular}




\begin{tabular}{|c|c|c|c|c|c|c|c|}
\hline Atom & Atom & Atom & Angle $/^{\circ}$ & Atom & Atom & Atom & Angle ${ }^{\circ}$ \\
\hline $\begin{array}{l}\mathrm{Si} 7 \\
\end{array}$ & $\mathrm{O} 10$ & Si1 & $136.9(2)$ & N2 & C90 & C91 & $123.2(6)$ \\
\hline $\mathrm{Si} 5$ & $\mathrm{O} 11$ & $\mathrm{Si} 7$ & $146.5(3)$ & $\mathrm{C} 92$ & C91 & $\mathrm{C} 90$ & $118.1(7)$ \\
\hline $\mathrm{Si} 5$ & $\mathrm{O} 12$ & $\mathrm{Si} 6$ & $148.3(3)$ & C93 & C92 & C91 & $119.4(7)$ \\
\hline Mn1 & $\mathrm{O} 13$ & Mn3 & $97.10(14)$ & $\mathrm{C} 92$ & C93 & C94 & $119.2(7)$ \\
\hline $\mathrm{Si} 9$ & $\mathrm{O} 13$ & Mn1 & $141.1(2)$ & $\mathrm{N} 2$ & C94 & C93 & $122.6(7)$ \\
\hline $\mathrm{Si} 9$ & $\mathrm{O} 13$ & $\mathrm{Mn} 3$ & $111.55(19)$ & N3 & C95 & C96 & $122.1(9)$ \\
\hline Mn3 & $\mathrm{O} 14$ & $\mathrm{Mn} 2$ & $101.63(14)$ & C97 & C96 & C95 & $118.8(9)$ \\
\hline Si10 & $\mathrm{O} 14$ & $\mathrm{Mn} 2$ & $118.33(18)$ & C96 & C97 & C98 & $120.2(10)$ \\
\hline Si10 & $\mathrm{O} 14$ & $\mathrm{Mn} 3$ & $131.50(19)$ & C97 & C98 & C99 & $115.4(9)$ \\
\hline Si8 & $\mathrm{O} 15$ & $\mathrm{Mn} 2$ & $153.6(2)$ & N3 & C99 & C98 & $124.6(10)$ \\
\hline Si14 & $\mathrm{O} 16$ & $\mathrm{Si} 9$ & $151.5(2)$ & N4 & $\mathrm{C} 100$ & $\mathrm{C} 101$ & $122.8(7)$ \\
\hline Si12 & $\mathrm{O} 17$ & $\mathrm{Si} 9$ & $164.0(3)$ & $\mathrm{C} 102$ & C101 & $\mathrm{C} 100$ & $117.7(8)$ \\
\hline Si13 & $\mathrm{O} 18$ & Si10 & $147.8(2)$ & C103 & C102 & C101 & $121.1(8)$ \\
\hline Si14 & O19 & Si10 & $139.4(2)$ & $\mathrm{C} 102$ & C103 & C104 & $117.6(9)$ \\
\hline Si13 & $\mathrm{O} 20$ & $\mathrm{Si} 8$ & $135.8(2)$ & N4 & C104 & C103 & $124.2(8)$ \\
\hline Si14 & $\mathrm{O} 21$ & Si11 & $141.0(2)$ & C108 & $\mathrm{O} 25$ & C105 & $110.7(12)$ \\
\hline Si11 & $\mathrm{O} 22$ & Si13 & $155.3(3)$ & C106 & C105 & $\mathrm{O} 25$ & $96.6(12)$ \\
\hline Si11 & $\mathrm{O} 23$ & Si12 & $141.2(3)$ & C105 & C106 & $\mathrm{C} 107$ & $115.0(14)$ \\
\hline Si12 & $\mathrm{O} 24$ & $\mathrm{Si} 8$ & $137.3(2)$ & C108 & C107 & C106 & $100.3(15)$ \\
\hline $\mathrm{C} 85$ & N1 & Mn1 & $121.7(4)$ & C107 & C108 & $\mathrm{O} 25$ & $107.2(13)$ \\
\hline C85 & N1 & C89 & $116.1(5)$ & C109 & $\mathrm{O} 26$ & C112 & $99.7(10)$ \\
\hline C89 & N1 & Mn1 & $122.1(4)$ & C110 & C109 & $\mathrm{O} 26$ & $103.5(12)$ \\
\hline C90 & N2 & $\mathrm{Mn} 2$ & $121.7(4)$ & C111 & C110 & C109 & $105.1(15)$ \\
\hline C94 & N2 & $\mathrm{Mn} 2$ & $120.9(4)$ & C110 & C111 & C112 & $113.5(14)$ \\
\hline C94 & N2 & C90 & $117.4(5)$ & C111 & C112 & $\mathrm{O} 26$ & $103.4(12)$ \\
\hline C95 & N3 & Mn3 & $121.1(5)$ & C116 & $\mathrm{O} 27$ & C113 & $109.4(15)$ \\
\hline C95 & N3 & C99 & $116.1(7)$ & $\mathrm{O} 27$ & C113 & C114 & $88.0(12)$ \\
\hline C99 & N3 & Mn3 & $122.2(6)$ & C115 & C114 & C113 & $114.6(14)$ \\
\hline C100 & N4 & Mn3 & $118.0(4)$ & C114 & C115 & C116 & $97.4(13)$ \\
\hline $\mathrm{C} 100$ & N4 & C104 & $116.7(6)$ & $\mathrm{O} 27$ & C116 & C115 & $104.6(14)$ \\
\hline C104 & N4 & Mn3 & $125.3(5)$ & & & & \\
\hline
\end{tabular}

\section{Experimental}

A single crystal of $\mathrm{C}_{116} \mathrm{H}_{114} \mathrm{Mn}_{3} \mathrm{~N}_{4} \mathrm{O}_{27} \mathrm{Si}_{14}$ was selected and mounted on a diffractometer. The crystal was kept at $99.93 \mathrm{~K}$ during data collection. The structure was solved with the ShelXS structure solution program using Structure Expansion and refined with the $\mathrm{XL}^{2}$ refinement package using Least Squares minimization, and Olex $2^{4}$ as a GUI. 


\section{Crystal structure determination}

Crystal Data for $\mathrm{C}_{116} \mathrm{H}_{114} \mathrm{Mn}_{3} \mathrm{~N}_{4} \mathrm{O}_{27} \mathrm{Si}_{14}(M=2554.19 \mathrm{~g} / \mathrm{mol}$ ): triclinic, space group P-1 (no. 2), $a=14.932(3) \AA, b=16.109(3) \AA, c=29.164(6) \AA, \alpha=75.886(4)^{\circ}, \beta=82.173(4)^{\circ}, \gamma=$ $72.330(4)^{\circ}, V=6468(2) \AA^{3}, Z=2, T=99.93 \mathrm{~K}, \mu(\mathrm{MoK} \alpha)=0.487 \mathrm{~mm}^{-1}$, Dcalc $=1.312 \mathrm{~g} / \mathrm{cm}^{3}$, 101139 reflections measured $\left(2.716^{\circ} \leq 2 \Theta \leq 52.992^{\circ}\right), 26323$ unique $\left(R_{\text {int }}=0.0563, \mathrm{R}_{\text {sigma }}=\right.$ $0.0601)$ which were used in all calculations. The final $R_{1}$ was 0.0829 (I $\left.>2 \sigma(\mathrm{I})\right)$ and $w R_{2}$ was 0.2287 (all data).

\section{Refinement model description}

Details:

Number of restraints - 970

1. Fixed Uiso

At 1.2 times of:

All $\mathrm{C}(\mathrm{H})$ groups, All $\mathrm{C}(\mathrm{H}, \mathrm{H})$ groups

2. Restrained distances

$\mathrm{O} 27-\mathrm{C} 113=\mathrm{O} 27-\mathrm{C} 116$

1.45 with sigma of 0.02

$\mathrm{C} 113-\mathrm{C} 114=\mathrm{C} 115-\mathrm{C} 114=\mathrm{C} 115-\mathrm{C} 116$

1.54 with sigma of 0.02

C105-C106

1.54 with sigma of 0.01

H11G-H87_\$1

2.4 with sigma of 0.02

C106-C107

1.54 with sigma of 0.01

H10C-H83_\$2

2.4 with sigma of 0.02

C113-C116

2.293 with sigma of 0.04

O27-C114

2.51526 with sigma of 0.04

$\mathrm{O} 27-\mathrm{C} 115$

2.51526 with sigma of 0.04

C116-C114

2.4423 with sigma of 0.04

C113-C115

2.4423 with sigma of 0.04

3. Rigid bond restraints

O25, C105, C106, C107, C108, O26, C109, C110, C111, C112, O27, C113, C114,

C115, C116

with sigma for 1-2 distances of 0.001 and sigma for 1-3 distances of 0.001

C7, C8, C9, C10, C11, C12, C13, C14, C15, C16, C17, C18, C31, C32, C33, C34, C35, C36, C80, C81, C82, C83, C84, C95, C96, C97, C98, C99

with sigma for 1-2 distances of 0.001 and sigma for 1-3 distances of 0.001

4. Uiso/Uaniso restraints and constraints

$\mathrm{O} 25 \approx \mathrm{C} 105 \approx \mathrm{C} 106 \approx \mathrm{C} 107 \approx \mathrm{C} 108 \approx \mathrm{O} 26 \approx \mathrm{C} 109 \approx \mathrm{C} 110$

$\approx \mathrm{C} 111 \approx \mathrm{C} 112 \approx \mathrm{O} 27 \approx \mathrm{C} 113 \approx \mathrm{C} 114 \approx \mathrm{C} 115 \approx \mathrm{C} 116$ : 
within $1.7 \mathrm{~A}$ with sigma of 0.004 and sigma for terminal atoms of 0.008

$\mathrm{C} 7 \approx \mathrm{C} 8 \approx \mathrm{C} 9 \approx \mathrm{C} 10 \approx \mathrm{C} 11 \approx \mathrm{C} 12 \approx \mathrm{C} 13 \approx \mathrm{C} 14 \approx$

$\mathrm{C} 15 \approx \mathrm{C} 16 \approx \mathrm{C} 17 \approx \mathrm{C} 18 \approx \mathrm{C} 31 \approx \mathrm{C} 32 \approx \mathrm{C} 33 \approx \mathrm{C} 34 \approx$

$\mathrm{C} 35 \approx \mathrm{C} 36 \approx \mathrm{C} 80 \approx \mathrm{C} 81 \approx \mathrm{C} 82 \approx \mathrm{C} 83 \approx \mathrm{C} 84 \approx \mathrm{C} 95 \approx$

$\mathrm{C} 96 \approx \mathrm{C} 97 \approx \mathrm{C} 98 \approx \mathrm{C} 99$ : within $1.7 \mathrm{~A}$ with sigma of 0.004 and sigma

for terminal atoms of 0.008

$\mathrm{C} 61 \approx \mathrm{C} 61 \mathrm{~A} \approx \mathrm{C} 62 \approx \mathrm{C} 62 \mathrm{~A} \approx \mathrm{C} 63 \approx \mathrm{C} 63 \mathrm{~A} \approx \mathrm{C} 64 \approx \mathrm{C} 64 \mathrm{~A}$

$\approx \mathrm{C} 65 \approx \mathrm{C} 65 \mathrm{~A} \approx \mathrm{C} 66 \approx \mathrm{C} 66 \mathrm{~A}$ : within $2 \mathrm{~A}$ with sigma of 0.01 and

sigma for terminal atoms of 0.02

$\mathrm{C} 31 \approx \mathrm{C} 31 \mathrm{~A} \approx \mathrm{C} 32 \approx \mathrm{C} 32 \mathrm{~A} \approx \mathrm{C} 33 \approx \mathrm{C} 33 \mathrm{~A} \approx \mathrm{C} 34 \approx \mathrm{C} 34 \mathrm{~A}$

$\approx \mathrm{C} 35 \approx \mathrm{C} 35 \mathrm{~A} \approx \mathrm{C} 36 \approx \mathrm{C} 36 \mathrm{~A}$ : within $2 \mathrm{~A}$ with sigma of 0.01 and

sigma for terminal atoms of 0.02

$\mathrm{C} 25 \approx \mathrm{C} 25 \mathrm{~A} \approx \mathrm{C} 26 \approx \mathrm{C} 26 \mathrm{~A} \approx \mathrm{C} 27 \approx \mathrm{C} 27 \mathrm{~A} \approx \mathrm{C} 28 \approx \mathrm{C} 28 \mathrm{~A}$

$\approx \mathrm{C} 29 \approx \mathrm{C} 29 \mathrm{~A} \approx \mathrm{C} 30 \approx \mathrm{C} 30 \mathrm{~A}$ : within $2 \mathrm{~A}$ with sigma of 0.01 and

sigma for terminal atoms of 0.02

$\operatorname{Uanis}(\mathrm{C} 31) \approx \operatorname{Ueq}, \operatorname{Uanis}(\mathrm{C} 32) \approx \operatorname{Ueq}, \operatorname{Uanis}(\mathrm{C} 33) \approx \operatorname{Ueq}, \operatorname{Uanis}(\mathrm{C} 34)$

$\approx \operatorname{Ueq}, \operatorname{Uanis}(\mathrm{C} 35) \approx \operatorname{Ueq}, \operatorname{Uanis}(\mathrm{C} 36) \approx \operatorname{Ueq}, \operatorname{Uanis}(\mathrm{C} 31 \mathrm{~A}) \approx \mathrm{Ueq}$

$\operatorname{Uanis}(\mathrm{C} 33 \mathrm{~A}) \approx \operatorname{Ueq}, \operatorname{Uanis}(\mathrm{C} 34 \mathrm{~A}) \approx \operatorname{Ueq}$, Uanis $(\mathrm{C} 35 \mathrm{~A}) \approx \operatorname{Ueq}$,

$\operatorname{Uanis}(\mathrm{C} 36 \mathrm{~A}) \approx \operatorname{Ueq}, \operatorname{Uanis}(\mathrm{C} 32 \mathrm{~A}) \approx \mathrm{Ueq}$ : with sigma of 0.01 and sigma for

terminal atoms of 0.02

$\operatorname{Uanis}(\mathrm{C} 70) \approx \operatorname{Ueq}, \operatorname{Uanis}(\mathrm{C} 69) \approx \operatorname{Ueq}, \operatorname{Uanis}(\mathrm{C} 68) \approx \operatorname{Ueq}, \operatorname{Uanis}(\mathrm{C} 72)$

$\approx$ Ueq, Uanis $(\mathrm{C} 67) \approx$ Ueq, Uanis $(\mathrm{C} 71) \approx$ Ueq: with sigma of 0.01 and

sigma for terminal atoms of 0.02

$\operatorname{Uanis}(\mathrm{O} 27) \approx \operatorname{Ueq}, \operatorname{Uanis}(\mathrm{C} 116) \approx \operatorname{Ueq}, \operatorname{Uanis}(\mathrm{C} 114) \approx \operatorname{Ueq}, \operatorname{Uanis}(\mathrm{C} 115)$

$\approx$ Ueq, Uanis $(\mathrm{C} 113) \approx$ Ueq: with sigma of 0.02 and sigma for terminal

atoms of 0.04

5. Others

$\operatorname{Sof}(\mathrm{C} 26 \mathrm{~A})=\operatorname{Sof}(\mathrm{H} 26 \mathrm{~A})=\operatorname{Sof}(\mathrm{C} 27 \mathrm{~A})=\operatorname{Sof}(\mathrm{H} 27 \mathrm{~A})=1-\mathrm{FVAR}(1)$

$\operatorname{Sof}(\mathrm{C} 30)=\operatorname{Sof}(\mathrm{H} 30)=\operatorname{Sof}(\mathrm{C} 29)=\operatorname{Sof}(\mathrm{H} 29)=$ FVAR $(1)$

$\operatorname{Sof}(C 28 A)=\operatorname{Sof}(H 28 A)=\operatorname{Sof}(C 29 A)=\operatorname{Sof}(H 29 A)=\operatorname{Sof}(C 30 A)=\operatorname{Sof}(H 30 A)=\operatorname{Sof}(C 25 A)=1-$

$\operatorname{FVAR}(2)$

$\operatorname{Sof}(\mathrm{C} 26)=\operatorname{Sof}(\mathrm{H} 26)=\operatorname{Sof}(\mathrm{C} 25)=\operatorname{Sof}(\mathrm{C} 28)=\operatorname{Sof}(\mathrm{H} 28)=\operatorname{Sof}(\mathrm{C} 27)=\operatorname{Sof}(\mathrm{H} 27)=\mathrm{FVAR}(2)$

$\operatorname{Sof}(\mathrm{C} 31 \mathrm{~A})=\operatorname{Sof}(\mathrm{C} 32 \mathrm{~A})=\operatorname{Sof}(\mathrm{H} 32 \mathrm{~A})=\operatorname{Sof}(\mathrm{C} 33 \mathrm{~A})=\operatorname{Sof}($ H33A $)=\operatorname{Sof}(\mathrm{C} 34 \mathrm{~A})=\operatorname{Sof}($ H34A $)=$

$\operatorname{Sof}(\mathrm{C} 35 \mathrm{~A})=\operatorname{Sof}(\mathrm{H} 35 \mathrm{~A})=\operatorname{Sof}(\mathrm{C} 36 \mathrm{~A})=\operatorname{Sof}(\mathrm{H} 36 \mathrm{~A})=1-\mathrm{FVAR}(3)$

$\operatorname{Sof}(\mathrm{C} 31)=\operatorname{Sof}(\mathrm{C} 32)=\operatorname{Sof}(H 32)=\operatorname{Sof}(\mathrm{C} 33)=\operatorname{Sof}(H 33)=\operatorname{Sof}(\mathrm{C} 34)=\operatorname{Sof}(H 34)=\operatorname{Sof}(\mathrm{C} 35)=$

Sof $($ H35 $)=\operatorname{Sof}(\mathrm{C} 36)=\operatorname{Sof}(H 36)=$ FVAR(3)

$\operatorname{Sof}(\mathrm{C} 61 \mathrm{~A})=\operatorname{Sof}(\mathrm{C} 66 \mathrm{~A})=\operatorname{Sof}(\mathrm{H} 66 \mathrm{~A})=\operatorname{Sof}(\mathrm{C} 65 \mathrm{~A})=\operatorname{Sof}(\mathrm{H} 65 \mathrm{~A})=\operatorname{Sof}(\mathrm{C} 64 \mathrm{~A})=\operatorname{Sof}(\mathrm{H} 64 \mathrm{~A})=$

$\operatorname{Sof}(\mathrm{C} 63 \mathrm{~A})=\operatorname{Sof}(\mathrm{H} 63 \mathrm{~A})=\operatorname{Sof}(\mathrm{C} 62 \mathrm{~A})=\operatorname{Sof}(\mathrm{H} 62 \mathrm{~A})=1-\mathrm{FVAR}(4)$

$\operatorname{Sof}(\mathrm{C61})=\operatorname{Sof}(\mathrm{C} 66)=\operatorname{Sof}(\mathrm{H} 66)=\operatorname{Sof}(\mathrm{C} 65)=\operatorname{Sof}(\mathrm{H} 65)=\operatorname{Sof}(\mathrm{C} 64)=\operatorname{Sof}(\mathrm{H} 64)=\operatorname{Sof}(\mathrm{C} 63)=$

$\operatorname{Sof}(\mathrm{H} 63)=\operatorname{Sof}(\mathrm{C} 62)=\operatorname{Sof}(\mathrm{H} 62)=\mathrm{FVAR}(4)$

6.a Secondary $\mathrm{CH} 2$ refined with riding coordinates:

C105(H10A,H10B), C106(H10C,H10D), C107(H10E,H10F), C108(H10G,H10H), C109(H10I, H10J), C110(H11A,H11B), C111(H11C,H11D), C112(H11E,H11F), C113(H11G,H11H),

C114(H11I,H11J), C115(H11K,H11L), C116(H11M,H11N)

6.b Aromatic/amide $\mathrm{H}$ refined with riding coordinates:

C2(H2), C3(H3), C4(H4), C5(H5), C6(H6), C8(H8), C9(H9), C10(H10), C11(H11), 
C12(H12), C14(H14), C15(H15), C16(H16), C17(H17), C18(H18), C20(H20), C21(H21), C22(H22), C23(H23), C24(H24), C26(H26), C30(H30), C29(H29), C28(H28),

C27(H27), C26A(H26A), C27A(H27A), C28A(H28A), C29A(H29A), C30A(H30A), $\mathrm{C} 32(\mathrm{H} 32)$,

C33(H33), C34(H34), C35(H35), C36(H36), C32A(H32A), C33A(H33A), C34A(H34A), C35A(H35A), C36A(H36A), C38(H38), C39(H39), C40(H40), C41(H41), C42(H42),

C44(H44), C45(H45), C46(H46), C47(H47), C48(H48), C50(H50), C51(H51), C52(H52), C53(H53), C54(H54), C56(H56), C57(H57), C58(H58), C59(H59), C60(H60),

C66(H66), C65(H65), C64(H64), C63(H63), C62(H62), C66A(H66A), C65A(H65A), C64A(H64A), C63A(H63A), C62A(H62A), C68(H68), C69(H69), C70(H70), C71(H71), C72(H72), C74(H74), C75(H75), C76(H76), C77(H77), C78(H78), C80(H80), C81(H81), C82(H82), C83(H83), C84(H84), C85(H85), C86(H86), C87(H87), C88(H88),

C89(H89), C90(H90), C91(H91), C92(H92), C93(H93), C94(H94), C95(H95), C96(H96), C97(H97), C98(H98), C99(H99), C100(H100), C101(H101), C102(H102), C103(H103), C104(H104)

6.c Fitted hexagon refined as free rotating group:

C26(C25,C30,C29,C28,C27), C26A(C27A,C28A,C29A,C30A,C25A), C31(C32,C33,C34,C35, C36), C31A(C32A,C33A,C34A,C35A,C36A), C61(C66,C65,C64,C63,C62), C61A(C66A,C65A, C64A,C63A,C62A)

This report has been created with Olex2, compiled on 2018.05 .29 svn.r3508 for OlexSys. 


\section{Compound 2}

Table S7 Crystal data and structure refinement for 2.

\begin{tabular}{|c|c|}
\hline Identification code & 2 \\
\hline Empirical formula & $\mathrm{C}_{92} \mathrm{H}_{80} \mathrm{Mn}_{3} \mathrm{~N}_{4} \mathrm{O}_{22} \mathrm{Si}_{12}$ \\
\hline Formula weight & 2095.50 \\
\hline Temperature/K & $173(2)$ \\
\hline Crystal system & monoclinic \\
\hline Space group & $\mathrm{P} 2 / \mathrm{n}$ \\
\hline $\mathrm{a} / \AA$ & $17.0311(19)$ \\
\hline $\mathrm{b} / \AA$ & $15.4115(17)$ \\
\hline $\mathrm{c} / \AA \AA$ & $21.108(2)$ \\
\hline$\alpha /{ }^{\circ}$ & 90 \\
\hline$\beta /{ }^{\circ}$ & $97.753(2)$ \\
\hline$\gamma /{ }^{\circ}$ & 90 \\
\hline Volume $/ \AA^{3}$ & $5489.6(10)$ \\
\hline $\mathrm{Z}$ & 2 \\
\hline$\rho_{\text {calc }} \mathrm{g} / \mathrm{cm}^{3}$ & 1.268 \\
\hline$\mu / \mathrm{mm}^{-1}$ & 0.535 \\
\hline $\mathrm{F}(000)$ & 2158.0 \\
\hline Crystal size $/ \mathrm{mm}^{3}$ & $0.229 \times 0.160 \times 0.099$ \\
\hline Radiation & $\operatorname{MoK} \alpha(\lambda=0.71073)$ \\
\hline $2 \Theta$ range for data collection ${ }^{\circ}$ & 2.642 to 43.932 \\
\hline Index ranges & $-17 \leq \mathrm{h} \leq 17,-16 \leq \mathrm{k} \leq 16,-22 \leq 1 \leq 22$ \\
\hline Reflections collected & 30856 \\
\hline Independent reflections & $6710\left[\mathrm{R}_{\mathrm{int}}=0.0564, \mathrm{R}_{\text {sigma }}=0.0451\right]$ \\
\hline Data/restraints/parameters & $6710 / 967 / 798$ \\
\hline Goodness-of-fit on $\mathrm{F}^{2}$ & 1.099 \\
\hline Final $R$ indexes $[\mathrm{I}>=2 \sigma(\mathrm{I})]$ & $\mathrm{R}_{1}=0.0811, \mathrm{wR}_{2}=0.1739$ \\
\hline Final R indexes [all data] & $\mathrm{R}_{1}=0.1140, \mathrm{wR}_{2}=0.1955$ \\
\hline Largest diff. peak/hole / e $\AA^{-3}$ & $0.48 /-0.47$ \\
\hline
\end{tabular}

Table S8. Bond Lengths for 2.

\begin{tabular}{llrllr}
\multicolumn{1}{c}{ Atom } & \multicolumn{1}{c}{ Atom } & \multicolumn{1}{c}{ Length $/ \AA$} & Atom & \multicolumn{1}{c}{ Atom } & Length/ \\
\hline $\mathrm{Mn} 1$ & $\mathrm{O} 1$ & $2.059(6)$ & $\mathrm{C} 16$ & $\mathrm{C} 17$ & 1.3900 \\
$\mathrm{Mn} 1$ & $\mathrm{O}^{1}$ & $2.059(6)$ & $\mathrm{C} 17$ & $\mathrm{C} 18$ & 1.3900 \\
$\mathrm{Mn} 1$ & $\mathrm{O} 11^{1}$ & $2.098(5)$ & $\mathrm{C} 13 \mathrm{~A}$ & $\mathrm{C} 14 \mathrm{~A}$ & 1.3900 \\
$\mathrm{Mn} 1$ & $\mathrm{O} 11$ & $2.098(5)$ & $\mathrm{C} 13 \mathrm{~A}$ & $\mathrm{C} 18 \mathrm{~A}$ & 1.3900 \\
$\mathrm{Mn} 1$ & $\mathrm{Si5}$ & $3.014(2)$ & $\mathrm{C} 14 \mathrm{~A}$ & $\mathrm{C} 15 \mathrm{~A}$ & 1.3900 \\
$\mathrm{Mn} 1$ & $\mathrm{Si5}{ }^{1}$ & $3.014(2)$ & $\mathrm{C} 15 \mathrm{~A}$ & $\mathrm{C} 16 \mathrm{~A}$ & 1.3900 \\
$\mathrm{Mn} 1$ & $\mathrm{Mn} 2$ & $3.0850(15)$ & $\mathrm{C} 16 \mathrm{~A}$ & $\mathrm{C} 17 \mathrm{~A}$ & 1.3900 \\
$\mathrm{Mn} 1$ & $\mathrm{Mn}{ }^{1}$ & $3.0851(15)$ & $\mathrm{C} 17 \mathrm{~A}$ & $\mathrm{C} 18 \mathrm{~A}$ & 1.3900 \\
$\mathrm{Mn} 2$ & $\mathrm{O} 3$ & $1.843(5)$ & $\mathrm{C} 19 \mathrm{~A}$ & $\mathrm{C} 24 \mathrm{~A}$ & 1.3900
\end{tabular}




\begin{tabular}{|c|c|c|c|c|c|}
\hline Atom & Atom & Length/Å & Atom & Atom & Length/Å \\
\hline $\mathrm{Mn} 2$ & $\mathrm{O} 10^{1}$ & $1.869(5)$ & C19A & $\mathrm{C} 20 \mathrm{~A}$ & 1.3900 \\
\hline $\mathrm{Mn} 2$ & O11 & $2.049(6)$ & $\mathrm{C} 24 \mathrm{~A}$ & $\mathrm{C} 23 \mathrm{~A}$ & 1.3900 \\
\hline $\mathrm{Mn} 2$ & $\mathrm{O} 1^{1}$ & $2.128(5)$ & $\mathrm{C} 23 \mathrm{~A}$ & $\mathrm{C} 22 \mathrm{~A}$ & 1.3900 \\
\hline $\mathrm{Mn} 2$ & N1 & $2.199(8)$ & $\mathrm{C} 22 \mathrm{~A}$ & $\mathrm{C} 21 \mathrm{~A}$ & 1.3900 \\
\hline $\mathrm{Mn} 2$ & $\mathrm{~N} 2$ & $2.238(7)$ & $\mathrm{C} 21 \mathrm{~A}$ & $\mathrm{C} 20 \mathrm{~A}$ & 1.3900 \\
\hline Si1 & $\mathrm{O} 3$ & $1.604(6)$ & $\mathrm{C} 20$ & $\mathrm{C} 21$ & 1.3900 \\
\hline Sil & $\mathrm{O} 2$ & $1.617(6)$ & $\mathrm{C} 20$ & C19 & 1.3900 \\
\hline Sil & O6 & $1.642(5)$ & $\mathrm{C} 21$ & $\mathrm{C} 22$ & 1.3900 \\
\hline Sil & $\mathrm{C} 1 \mathrm{~A}$ & $1.878(12)$ & $\mathrm{C} 22$ & $\mathrm{C} 23$ & 1.3900 \\
\hline Sil & $\mathrm{C} 1$ & 1.899(9) & $\mathrm{C} 23$ & $\mathrm{C} 24$ & 1.3900 \\
\hline $\mathrm{Si} 2$ & O1 & $1.592(5)$ & $\mathrm{C} 24$ & C19 & 1.3900 \\
\hline $\mathrm{Si} 2$ & $\mathrm{O} 2$ & $1.624(6)$ & $\mathrm{C} 25$ & $\mathrm{C} 26$ & 1.3900 \\
\hline $\mathrm{Si} 2$ & $\mathrm{O} 4$ & $1.638(6)$ & $\mathrm{C} 25$ & $\mathrm{C} 30$ & 1.3900 \\
\hline $\mathrm{Si} 2$ & C7A & $1.847(16)$ & $\mathrm{C} 26$ & $\mathrm{C} 27$ & 1.3900 \\
\hline $\mathrm{Si} 2$ & $\mathrm{C} 7$ & $1.899(16)$ & $\mathrm{C} 27$ & $\mathrm{C} 28$ & 1.3900 \\
\hline $\mathrm{Si} 3$ & $\mathrm{O} 7$ & $1.613(5)$ & $\mathrm{C} 28$ & C29 & 1.3900 \\
\hline $\mathrm{Si} 3$ & $\mathrm{O} 4$ & $1.625(6)$ & $\mathrm{C} 29$ & C30 & 1.3900 \\
\hline $\mathrm{Si} 3$ & O5 & $1.635(7)$ & $\mathrm{C} 25 \mathrm{~A}$ & C30A & $1.35(3)$ \\
\hline $\mathrm{Si} 3$ & C13A & $1.78(3)$ & $\mathrm{C} 25 \mathrm{~A}$ & $\mathrm{C} 26 \mathrm{~A}$ & $1.39(3)$ \\
\hline $\mathrm{Si} 3$ & C13 & $1.885(9)$ & $\mathrm{C} 26 \mathrm{~A}$ & C27A & $1.37(2)$ \\
\hline $\mathrm{Si} 4$ & $\mathrm{O} 6$ & $1.611(6)$ & C27A & C28A & $1.36(4)$ \\
\hline $\mathrm{Si} 4$ & O9 & $1.618(5)$ & C28A & C29A & $1.36(4)$ \\
\hline $\mathrm{Si} 4$ & O5 & $1.626(7)$ & C29A & $\mathrm{C} 30 \mathrm{~A}$ & $1.41(3)$ \\
\hline $\mathrm{Si} 4$ & C19 & $1.854(9)$ & C31 & C32 & 1.3900 \\
\hline $\mathrm{Si} 4$ & C19A & $1.892(12)$ & C31 & $\mathrm{C} 36$ & 1.3900 \\
\hline $\mathrm{Si} 5$ & O11 & $1.603(6)$ & C32 & C33 & 1.3900 \\
\hline $\mathrm{Si} 5$ & O8 & $1.615(7)$ & C33 & C34 & 1.3900 \\
\hline $\mathrm{Si} 5$ & O9 & $1.625(5)$ & C34 & C35 & 1.3900 \\
\hline $\mathrm{Si} 5$ & $\mathrm{C} 25$ & $1.824(8)$ & C35 & C36 & 1.3900 \\
\hline $\mathrm{Si} 5$ & $\mathrm{C} 25 \mathrm{~A}$ & $1.95(2)$ & C31A & C32A & 1.3900 \\
\hline $\mathrm{Si} 6$ & $\mathrm{O} 10$ & $1.585(6)$ & C31A & C36A & 1.3900 \\
\hline $\mathrm{Si} 6$ & $\mathrm{O} 7$ & $1.638(6)$ & C32A & C33A & 1.3900 \\
\hline $\mathrm{Si} 6$ & $\mathrm{O} 8$ & $1.651(7)$ & C33A & C34A & 1.3900 \\
\hline $\mathrm{Si} 6$ & C31A & $1.842(11)$ & C34A & $\mathrm{C} 35 \mathrm{~A}$ & 1.3900 \\
\hline $\mathrm{Si} 6$ & C31 & $1.964(12)$ & C35A & C36A & 1.3900 \\
\hline N1 & $\mathrm{C} 41$ & $1.336(13)$ & C37 & C38 & $1.396(16)$ \\
\hline N1 & C37 & $1.338(12)$ & C38 & C39 & $1.368(17)$ \\
\hline N2 & $\mathrm{C} 46$ & $1.326(13)$ & C39 & $\mathrm{C} 40$ & $1.354(16)$ \\
\hline N2 & $\mathrm{C} 42$ & $1.335(11)$ & $\mathrm{C} 40$ & $\mathrm{C} 41$ & $1.387(15)$ \\
\hline $\mathrm{C} 7$ & $\mathrm{C} 8$ & 1.3900 & $\mathrm{C} 42$ & $\mathrm{C} 43$ & $1.401(13)$ \\
\hline $\mathrm{C} 7$ & $\mathrm{C} 12$ & 1.3900 & $\mathrm{C} 43$ & $\mathrm{C} 44$ & $1.368(16)$ \\
\hline
\end{tabular}




\begin{tabular}{llrllr}
\multicolumn{1}{c}{ Atom } & Atom & Length $/ \AA$ & Atom & Atom & Length $/ \AA$ \\
\hline C8 & C9 & 1.3900 & C44 & C45 & $1.350(15)$ \\
C9 & C10 & 1.3900 & C45 & C46 & $1.395(14)$ \\
C10 & C11 & 1.3900 & C1 & C5 & 1.3900 \\
C11 & C12 & 1.3900 & C1 & C3 & 1.3900 \\
C7A & C8A & 1.3900 & C5 & C6 & 1.3900 \\
C7A & C12A & 1.3900 & C6 & C4 & 1.3900 \\
C8A & C9A & 1.3900 & C4 & C2 & 1.3900 \\
C9A & C10A & 1.3900 & C2 & C3 & 1.3900 \\
C10A & C11A & 1.3900 & C1A & C5A & 1.3900 \\
C11A & C12A & 1.3900 & C1A & C3A & 1.3900 \\
C13 & C14 & 1.3900 & C5A & C6A & 1.3900 \\
C13 & C18 & 1.3900 & C6A & C4A & 1.3900 \\
C14 & C15 & 1.3900 & C4A & C2A & 1.3900 \\
C15 & C16 & 1.3900 & C2A & C3A & 1.3900 \\
\hline
\end{tabular}

13/2-X,+Y,1/2-Z

Table S9. Bond Angles for 2.

\begin{tabular}{|c|c|c|c|c|c|c|c|}
\hline Atom & Atom & Atom & Angle $/^{\circ}$ & Atom & Atom & Atom & Angle $/^{\circ}$ \\
\hline $\mathrm{O} 1$ & Mn1 & $\mathrm{O} 1^{1}$ & $116.0(3)$ & $\mathrm{Si} 4$ & O9 & $\mathrm{Si} 5$ & $140.1(3)$ \\
\hline $\mathrm{O} 1$ & Mn1 & $\mathrm{O} 11^{1}$ & $84.7(2)$ & Si6 & $\mathrm{O} 10$ & $\mathrm{Mn} 2^{1}$ & $137.3(4)$ \\
\hline $\mathrm{O} 1^{1}$ & Mn1 & $\mathrm{O} 11^{1}$ & $123.6(2)$ & Si5 & O11 & $\mathrm{Mn} 2$ & $155.2(4)$ \\
\hline $\mathrm{O} 1$ & Mn1 & O11 & $123.6(2)$ & Si5 & O11 & Mn1 & $108.3(3)$ \\
\hline $\mathrm{O} 1^{1}$ & Mn1 & O11 & $84.7(2)$ & $\mathrm{Mn} 2$ & O11 & Mn1 & $96.2(2)$ \\
\hline $\mathrm{O} 11^{1}$ & Mn1 & O11 & $128.4(3)$ & $\mathrm{C} 41$ & N1 & C37 & $118.4(10)$ \\
\hline O1 & Mn1 & Si5 & $107.06(15)$ & $\mathrm{C} 41$ & N1 & $\mathrm{Mn} 2$ & $120.6(8)$ \\
\hline $\mathrm{O} 1^{1}$ & Mn1 & Si5 & $115.01(15)$ & C37 & N1 & $\mathrm{Mn} 2$ & $120.9(8)$ \\
\hline $\mathrm{O} 11^{1}$ & Mn1 & Si5 & $105.97(14)$ & C46 & $\mathrm{N} 2$ & $\mathrm{C} 42$ & $116.5(8)$ \\
\hline O11 & Mn1 & Si5 & $30.34(17)$ & $\mathrm{C} 46$ & $\mathrm{~N} 2$ & $\mathrm{Mn} 2$ & $120.9(6)$ \\
\hline $\mathrm{O} 1$ & Mn1 & $\mathrm{Si} 5^{1}$ & $115.01(15)$ & $\mathrm{C} 42$ & $\mathrm{~N} 2$ & $\mathrm{Mn} 2$ & $122.2(7)$ \\
\hline $\mathrm{O} 1^{1}$ & Mn1 & $\mathrm{Si}^{1}$ & $107.06(15)$ & $\mathrm{C} 8$ & $\mathrm{C} 7$ & $\mathrm{C} 12$ & 120.0 \\
\hline $\mathrm{O} 11^{1}$ & Mn1 & $\mathrm{Si} 5^{1}$ & $30.35(17)$ & $\mathrm{C} 8$ & $\mathrm{C} 7$ & $\mathrm{Si} 2$ & $118.9(11)$ \\
\hline O11 & Mn1 & $\mathrm{Si} 5^{1}$ & $105.97(14)$ & $\mathrm{C} 12$ & $\mathrm{C} 7$ & $\mathrm{Si} 2$ & $120.9(11)$ \\
\hline Si5 & Mn1 & $\mathrm{Si} 5^{1}$ & $95.00(9)$ & C9 & $\mathrm{C} 8$ & $\mathrm{C} 7$ & 120.0 \\
\hline $\mathrm{O} 1$ & Mn1 & $\mathrm{Mn} 2$ & $133.81(14)$ & $\mathrm{C} 8$ & C9 & $\mathrm{C} 10$ & 120.0 \\
\hline $\mathrm{O} 1^{1}$ & Mn1 & Mn2 & $43.41(14)$ & $\mathrm{C} 11$ & $\mathrm{C} 10$ & C9 & 120.0 \\
\hline $\mathrm{O} 11^{1}$ & Mn1 & $\mathrm{Mn} 2$ & $141.18(17)$ & $\mathrm{C} 10$ & $\mathrm{C} 11$ & $\mathrm{C} 12$ & 120.0 \\
\hline O11 & Mn1 & $\mathrm{Mn} 2$ & $41.32(17)$ & $\mathrm{C} 11$ & $\mathrm{C} 12$ & $\mathrm{C} 7$ & 120.0 \\
\hline Si5 & Mn1 & $\mathrm{Mn} 2$ & $71.61(6)$ & $\mathrm{C} 8 \mathrm{~A}$ & C7A & $\mathrm{C} 12 \mathrm{~A}$ & 120.0 \\
\hline $\mathrm{Si} 5^{1}$ & Mn1 & $\mathrm{Mn} 2$ & $111.04(7)$ & C8A & C7A & $\mathrm{Si} 2$ & $122.5(11)$ \\
\hline $\mathrm{O} 1$ & Mn1 & $\mathrm{Mn} 2^{1}$ & $43.41(14)$ & $\mathrm{C} 12 \mathrm{~A}$ & C7A & $\mathrm{Si} 2$ & $117.4(11)$ \\
\hline
\end{tabular}




\begin{tabular}{|c|c|c|c|c|c|c|c|}
\hline Atom & Atom & Atom & Angle $/^{\circ}$ & Atom & Atom & Atom & Angle $/^{\circ}$ \\
\hline$\overline{\mathrm{O} 1}{ }^{1}$ & Mn1 & $\mathrm{Mn} 2^{1}$ & $133.81(14)$ & C9A & $\mathrm{C} 8 \mathrm{~A}$ & C7A & 120.0 \\
\hline $\mathrm{O} 11^{1}$ & Mn1 & $\operatorname{Mn} 2^{1}$ & $41.31(17)$ & $\mathrm{C} 8 \mathrm{~A}$ & C9A & $\mathrm{C} 10 \mathrm{~A}$ & 120.0 \\
\hline $\mathrm{O} 11$ & Mn1 & $\mathrm{Mn} 2^{1}$ & $141.18(17)$ & C9A & $\mathrm{C} 10 \mathrm{~A}$ & C11A & 120.0 \\
\hline $\mathrm{Si} 5$ & Mn1 & $\operatorname{Mn} 2^{1}$ & $111.04(7)$ & $\mathrm{C} 12 \mathrm{~A}$ & $\mathrm{C} 11 \mathrm{~A}$ & C10A & 120.0 \\
\hline $\mathrm{Si}^{1}$ & Mn1 & $\mathrm{Mn} 2^{1}$ & $71.61(6)$ & C11A & $\mathrm{C} 12 \mathrm{~A}$ & C7A & 120.0 \\
\hline $\mathrm{Mn} 2$ & Mn1 & $\mathrm{Mn} 2^{1}$ & $176.31(7)$ & $\mathrm{C} 14$ & C13 & $\mathrm{C} 18$ & 120.0 \\
\hline $\mathrm{O} 3$ & $\mathrm{Mn} 2$ & $\mathrm{O} 10^{1}$ & $174.4(3)$ & $\mathrm{C} 14$ & $\mathrm{C} 13$ & $\mathrm{Si} 3$ & $121.4(7)$ \\
\hline $\mathrm{O} 3$ & Mn2 & $\mathrm{O} 11$ & $91.6(2)$ & $\mathrm{C} 18$ & $\mathrm{C} 13$ & $\mathrm{Si} 3$ & $118.6(7)$ \\
\hline $\mathrm{O} 10^{1}$ & $\mathrm{Mn} 2$ & $\mathrm{O} 11$ & $92.3(2)$ & $\mathrm{C} 13$ & $\mathrm{C} 14$ & $\mathrm{C} 15$ & 120.0 \\
\hline $\mathrm{O} 3$ & $\mathrm{Mn} 2$ & $\mathrm{O} 1^{1}$ & $94.0(2)$ & $\mathrm{C} 16$ & $\mathrm{C} 15$ & $\mathrm{C} 14$ & 120.0 \\
\hline $\mathrm{O} 10^{1}$ & Mn2 & $\mathrm{O} 1^{1}$ & $90.4(2)$ & $\mathrm{C} 15$ & $\mathrm{C} 16$ & $\mathrm{C} 17$ & 120.0 \\
\hline $\mathrm{O} 11$ & $\mathrm{Mn} 2$ & $\mathrm{O} 1^{1}$ & $84.2(2)$ & $\mathrm{C} 16$ & $\mathrm{C} 17$ & $\mathrm{C} 18$ & 120.0 \\
\hline $\mathrm{O} 3$ & Mn2 & N1 & $86.6(3)$ & $\mathrm{C} 17$ & C18 & C13 & 120.0 \\
\hline $\mathrm{O} 10^{1}$ & Mn2 & N1 & $89.7(3)$ & C14A & C13A & C18A & 120.0 \\
\hline $\mathrm{O} 11$ & $\mathrm{Mn} 2$ & N1 & $175.9(2)$ & C14A & C13A & $\mathrm{Si} 3$ & $119(2)$ \\
\hline $\mathrm{O} 1^{1}$ & $\mathrm{Mn} 2$ & N1 & $92.3(2)$ & C18A & C13A & $\mathrm{Si} 3$ & $120(2)$ \\
\hline $\mathrm{O} 3$ & $\mathrm{Mn} 2$ & $\mathrm{~N} 2$ & $89.2(3)$ & C13A & C14A & C15A & 120.0 \\
\hline $\mathrm{O} 10^{1}$ & $\mathrm{Mn} 2$ & N2 & $86.5(3)$ & C16A & C15A & C14A & 120.0 \\
\hline O11 & Mn2 & N2 & $93.7(3)$ & C15A & C16A & C17A & 120.0 \\
\hline $\mathrm{O} 1^{1}$ & $\mathrm{Mn} 2$ & $\mathrm{~N} 2$ & $176.2(2)$ & C18A & C17A & C16A & 120.0 \\
\hline N1 & $\mathrm{Mn} 2$ & N2 & $89.9(3)$ & C17A & C18A & C13A & 120.0 \\
\hline $\mathrm{O} 3$ & $\mathrm{Mn} 2$ & Mn1 & $95.31(19)$ & C24A & C19A & C20A & 120.0 \\
\hline $\mathrm{O} 10^{1}$ & Mn2 & Mn1 & $90.3(2)$ & C24A & C19A & $\mathrm{Si} 4$ & $114.8(10)$ \\
\hline $\mathrm{O} 11$ & Mn2 & Mn1 & $42.53(15)$ & C20A & C19A & $\mathrm{Si} 4$ & $125.2(10)$ \\
\hline $\mathrm{O} 1^{1}$ & $\mathrm{Mn} 2$ & Mn1 & $41.67(15)$ & C19A & $\mathrm{C} 24 \mathrm{~A}$ & $\mathrm{C} 23 \mathrm{~A}$ & 120.0 \\
\hline N1 & $\mathrm{Mn} 2$ & Mn1 & $133.97(18)$ & C22A & $\mathrm{C} 23 \mathrm{~A}$ & C24A & 120.0 \\
\hline N2 & $\mathrm{Mn} 2$ & Mn1 & $136.0(2)$ & $\mathrm{C} 21 \mathrm{~A}$ & $\mathrm{C} 22 \mathrm{~A}$ & $\mathrm{C} 23 \mathrm{~A}$ & 120.0 \\
\hline $\mathrm{O} 3$ & Sil & $\mathrm{O} 2$ & $112.4(3)$ & C22A & $\mathrm{C} 21 \mathrm{~A}$ & C20A & 120.0 \\
\hline $\mathrm{O} 3$ & Sil & O6 & $111.5(3)$ & $\mathrm{C} 21 \mathrm{~A}$ & $\mathrm{C} 20 \mathrm{~A}$ & C19A & 120.0 \\
\hline $\mathrm{O} 2$ & Si1 & O6 & $107.7(3)$ & C21 & $\mathrm{C} 20$ & C19 & 120.0 \\
\hline $\mathrm{O} 3$ & Sil & $\mathrm{C} 1 \mathrm{~A}$ & $107.8(7)$ & $\mathrm{C} 20$ & $\mathrm{C} 21$ & $\mathrm{C} 22$ & 120.0 \\
\hline $\mathrm{O} 2$ & Sil & $\mathrm{C} 1 \mathrm{~A}$ & $110.6(6)$ & $\mathrm{C} 23$ & $\mathrm{C} 22$ & $\mathrm{C} 21$ & 120.0 \\
\hline O6 & Sil & $\mathrm{C} 1 \mathrm{~A}$ & $106.7(6)$ & $\mathrm{C} 22$ & $\mathrm{C} 23$ & $\mathrm{C} 24$ & 120.0 \\
\hline $\mathrm{O} 3$ & Sil & $\mathrm{C} 1$ & $114.6(5)$ & $\mathrm{C} 23$ & $\mathrm{C} 24$ & C19 & 120.0 \\
\hline $\mathrm{O} 2$ & Sil & $\mathrm{C} 1$ & $108.4(4)$ & $\mathrm{C} 24$ & C19 & $\mathrm{C} 20$ & 120.0 \\
\hline O6 & Sil & $\mathrm{C} 1$ & $101.6(5)$ & $\mathrm{C} 24$ & C19 & $\mathrm{Si} 4$ & $119.0(8)$ \\
\hline O1 & $\mathrm{Si} 2$ & $\mathrm{O} 2$ & $106.4(3)$ & $\mathrm{C} 20$ & C19 & $\mathrm{Si} 4$ & $121.0(8)$ \\
\hline $\mathrm{O} 1$ & $\mathrm{Si} 2$ & O4 & $111.6(3)$ & $\mathrm{C} 26$ & $\mathrm{C} 25$ & $\mathrm{C} 30$ & 120.0 \\
\hline $\mathrm{O} 2$ & $\mathrm{Si} 2$ & $\mathrm{O} 4$ & $108.6(3)$ & $\mathrm{C} 26$ & $\mathrm{C} 25$ & $\mathrm{Si} 5$ & $115.3(6)$ \\
\hline $\mathrm{O} 1$ & $\mathrm{Si} 2$ & C7A & $113.6(6)$ & $\mathrm{C} 30$ & $\mathrm{C} 25$ & $\mathrm{Si} 5$ & $124.6(6)$ \\
\hline $\mathrm{O} 2$ & $\mathrm{Si} 2$ & $\mathrm{C} 7 \mathrm{~A}$ & $111.2(6)$ & $\mathrm{C} 27$ & $\mathrm{C} 26$ & $\mathrm{C} 25$ & 120.0 \\
\hline
\end{tabular}




\begin{tabular}{|c|c|c|c|c|c|c|c|}
\hline Atom & Atom & Atom & Angle $/^{\circ}$ & Atom & Atom & Atom & Angle ${ }^{\circ}$ \\
\hline $\mathrm{O} 4$ & $\mathrm{Si} 2$ & $\mathrm{C} 7 \mathrm{~A}$ & $105.4(6)$ & $\mathrm{C} 26$ & $\mathrm{C} 27$ & $\mathrm{C} 28$ & 120.0 \\
\hline $\mathrm{O} 1$ & $\mathrm{Si} 2$ & $\mathrm{C} 7$ & $115.6(7)$ & $\mathrm{C} 27$ & $\mathrm{C} 28$ & $\mathrm{C} 29$ & 120.0 \\
\hline $\mathrm{O} 2$ & $\mathrm{Si} 2$ & $\mathrm{C} 7$ & $106.1(6)$ & $\mathrm{C} 30$ & $\mathrm{C} 29$ & $\mathrm{C} 28$ & 120.0 \\
\hline $\mathrm{O} 4$ & $\mathrm{Si} 2$ & $\mathrm{C} 7$ & $108.2(6)$ & $\mathrm{C} 29$ & $\mathrm{C} 30$ & $\mathrm{C} 25$ & 120.0 \\
\hline $\mathrm{O} 7$ & $\mathrm{Si} 3$ & $\mathrm{O} 4$ & $110.6(3)$ & $\mathrm{C} 30 \mathrm{~A}$ & $\mathrm{C} 25 \mathrm{~A}$ & C26A & $116.8(18)$ \\
\hline $\mathrm{O} 7$ & $\mathrm{Si} 3$ & O5 & $109.4(3)$ & $\mathrm{C} 30 \mathrm{~A}$ & $\mathrm{C} 25 \mathrm{~A}$ & $\mathrm{Si} 5$ & $116(2)$ \\
\hline $\mathrm{O} 4$ & $\mathrm{Si} 3$ & O5 & $109.1(3)$ & $\mathrm{C} 26 \mathrm{~A}$ & $\mathrm{C} 25 \mathrm{~A}$ & $\mathrm{Si} 5$ & $127.2(18)$ \\
\hline $\mathrm{O} 7$ & $\mathrm{Si} 3$ & C13A & $103.4(14)$ & $\mathrm{C} 27 \mathrm{~A}$ & $\mathrm{C} 26 \mathrm{~A}$ & $\mathrm{C} 25 \mathrm{~A}$ & $123.4(19)$ \\
\hline $\mathrm{O} 4$ & $\mathrm{Si} 3$ & C13A & $116.9(14)$ & $\mathrm{C} 28 \mathrm{~A}$ & $\mathrm{C} 27 \mathrm{~A}$ & C26A & $118(2)$ \\
\hline $\mathrm{O} 5$ & $\mathrm{Si} 3$ & C13A & $107.1(13)$ & $\mathrm{C} 27 \mathrm{~A}$ & C28A & C29A & $121(2)$ \\
\hline $\mathrm{O} 7$ & $\mathrm{Si} 3$ & C13 & $110.8(5)$ & $\mathrm{C} 28 \mathrm{~A}$ & C29A & C30A & $119(2)$ \\
\hline $\mathrm{O} 4$ & $\mathrm{Si} 3$ & $\mathrm{C} 13$ & $105.9(4)$ & $\mathrm{C} 25 \mathrm{~A}$ & C30A & C29A & $121(2)$ \\
\hline $\mathrm{O} 5$ & $\mathrm{Si} 3$ & $\mathrm{C} 13$ & $111.0(4)$ & C32 & C31 & C36 & 120.0 \\
\hline O6 & $\mathrm{Si} 4$ & O9 & $110.0(3)$ & C32 & C31 & $\mathrm{Si} 6$ & 113.1(9) \\
\hline O6 & $\mathrm{Si} 4$ & O5 & $109.7(3)$ & C36 & C31 & Si6 & $126.4(9)$ \\
\hline O9 & $\mathrm{Si} 4$ & O5 & $109.5(3)$ & C33 & C32 & C31 & 120.0 \\
\hline O6 & $\mathrm{Si} 4$ & C19 & $110.7(6)$ & C34 & C33 & C32 & 120.0 \\
\hline O9 & $\mathrm{Si} 4$ & C19 & $110.9(5)$ & C33 & C34 & C35 & 120.0 \\
\hline $\mathrm{O} 5$ & $\mathrm{Si} 4$ & C19 & $106.0(6)$ & C36 & C35 & C34 & 120.0 \\
\hline O6 & $\mathrm{Si} 4$ & C19A & $105.4(7)$ & C35 & C36 & C31 & 120.0 \\
\hline O9 & $\mathrm{Si} 4$ & C19A & $107.4(6)$ & C32A & C31A & C36A & 120.0 \\
\hline O5 & $\mathrm{Si} 4$ & C19A & $114.7(7)$ & C32A & C31A & Si6 & $119.3(8)$ \\
\hline O11 & $\mathrm{Si} 5$ & O8 & $103.7(3)$ & C36A & C31A & Si6 & $120.4(8)$ \\
\hline O11 & $\mathrm{Si} 5$ & O9 & $112.0(3)$ & C31A & C32A & C33A & 120.0 \\
\hline O8 & $\mathrm{Si} 5$ & O9 & $109.6(4)$ & C32A & C33A & C34A & 120.0 \\
\hline O11 & $\mathrm{Si} 5$ & $\mathrm{C} 25$ & $121.1(6)$ & $\mathrm{C} 33 \mathrm{~A}$ & C34A & C35A & 120.0 \\
\hline O8 & $\mathrm{Si} 5$ & $\mathrm{C} 25$ & $105.9(4)$ & C36A & $\mathrm{C} 35 \mathrm{~A}$ & C34A & 120.0 \\
\hline O9 & $\mathrm{Si} 5$ & $\mathrm{C} 25$ & $104.1(5)$ & $\mathrm{C} 35 \mathrm{~A}$ & C36A & C31A & 120.0 \\
\hline O11 & $\mathrm{Si} 5$ & $\mathrm{C} 25 \mathrm{~A}$ & $109.9(10)$ & N1 & C37 & C38 & $121.9(11)$ \\
\hline O8 & $\mathrm{Si} 5$ & $\mathrm{C} 25 \mathrm{~A}$ & $113.7(8)$ & C39 & C38 & C37 & $118.2(12)$ \\
\hline O9 & Si5 & $\mathrm{C} 25 \mathrm{~A}$ & $107.9(8)$ & $\mathrm{C} 40$ & C39 & C38 & $120.5(14)$ \\
\hline O11 & $\mathrm{Si} 5$ & Mn1 & $41.4(2)$ & C39 & $\mathrm{C} 40$ & C41 & $118.7(13)$ \\
\hline O8 & $\mathrm{Si} 5$ & Mn1 & $62.3(2)$ & N1 & $\mathrm{C} 41$ & $\mathrm{C} 40$ & $122.3(12)$ \\
\hline O9 & $\mathrm{Si} 5$ & Mn1 & $125.2(2)$ & $\mathrm{N} 2$ & $\mathrm{C} 42$ & $\mathrm{C} 43$ & $122.4(10)$ \\
\hline $\mathrm{C} 25$ & $\mathrm{Si} 5$ & Mn1 & $130.7(5)$ & $\mathrm{C} 44$ & $\mathrm{C} 43$ & $\mathrm{C} 42$ & $118.2(10)$ \\
\hline $\mathrm{C} 25 \mathrm{~A}$ & $\mathrm{Si} 5$ & Mn1 & $125.3(8)$ & $\mathrm{C} 45$ & $\mathrm{C} 44$ & $\mathrm{C} 43$ & $121.1(11)$ \\
\hline $\mathrm{O} 10$ & $\mathrm{Si} 6$ & $\mathrm{O} 7$ & $111.2(3)$ & $\mathrm{C} 44$ & $\mathrm{C} 45$ & $\mathrm{C} 46$ & $116.4(12)$ \\
\hline $\mathrm{O} 10$ & Si6 & O8 & $112.7(3)$ & N2 & $\mathrm{C} 46$ & $\mathrm{C} 45$ & $125.3(11)$ \\
\hline $\mathrm{O} 7$ & $\mathrm{Si} 6$ & O8 & $107.3(3)$ & $\mathrm{C} 5$ & $\mathrm{C} 1$ & $\mathrm{C} 3$ & 120.0 \\
\hline $\mathrm{O} 10$ & $\mathrm{Si} 6$ & C31A & $112.2(5)$ & $\mathrm{C} 5$ & $\mathrm{C} 1$ & Sil & $119.8(7)$ \\
\hline $\mathrm{O} 7$ & Si6 & C31A & $111.0(5)$ & $\mathrm{C} 3$ & $\mathrm{C} 1$ & Sil & $120.1(7)$ \\
\hline
\end{tabular}




\begin{tabular}{lllrlllr}
\multicolumn{1}{c}{ Atom } & Atom & Atom & \multicolumn{1}{c}{ Angle $^{\circ}$} & Atom & Atom & Atom & Angle $^{\circ}$ \\
\hline O8 & Si6 & C31A & $101.9(5)$ & C6 & C5 & C1 & 120.0 \\
O10 & Si6 & C31 & $108.1(6)$ & C5 & C6 & C4 & 120.0 \\
O7 & Si6 & C31 & $99.0(5)$ & C2 & C4 & C6 & 120.0 \\
O8 & Si6 & C31 & $117.7(6)$ & C4 & C2 & C3 & 120.0 \\
Si2 & O1 & Mn1 & $114.0(3)$ & C2 & C3 & C1 & 120.0 \\
Si2 & O1 & Mn2 & $150.1(4)$ & C5A & C1A & C3A & 120.0 \\
Mn1 & O1 & Mn2 & $94.9(2)$ & C5A & C1A & Si1 & $122.8(10)$ \\
Si1 & O2 & Si2 & $164.3(4)$ & C3A & C1A & Si1 & $117.2(10)$ \\
Si1 & O3 & Mn2 & $133.8(4)$ & C1A & C5A & C6A & 120.0 \\
Si3 & O4 & Si2 & $137.4(4)$ & C4A & C6A & C5A & 120.0 \\
Si4 & O5 & Si3 & $145.2(4)$ & C6A & C4A & C2A & 120.0 \\
Si4 & O6 & Si1 & $142.4(4)$ & C4A & C2A & C3A & 120.0 \\
Si3 & O7 & Si6 & $147.6(4)$ & C2A & C3A & C1A & 120.0 \\
Si5 & O8 & Si6 & $150.2(4)$ & & & & \\
\hline
\end{tabular}

${ }^{13} / 2-\mathrm{X},+\mathrm{Y}, 1 / 2-\mathrm{Z}$

\section{Experimental}

A single crystal of $\mathrm{C}_{92} \mathrm{H}_{80} \mathrm{Mn}_{3} \mathrm{~N}_{4} \mathrm{O}_{22} \mathrm{Si}_{12}$ was selected and mounted on a diffractometer. The crystal was kept at $173(2) \mathrm{K}$ during data collection. Using Olex2 ${ }^{4}$ as a GUI, the structure was solved with the ShelXS structure solution program using Structure Expansion and refined with the $\mathrm{XL}^{2}$ refinement package using Least Squares minimisation.

\section{Crystal structure determination}

Crystal Data for $\mathrm{C}_{92} \mathrm{H}_{80} \mathrm{Mn}_{3} \mathrm{~N}_{4} \mathrm{O}_{22} \mathrm{Si}_{12}(M=2095.50 \mathrm{~g} / \mathrm{mol})$ : monoclinic, space group P2/n (no. 13), $a=17.0311(19) \AA, b=15.4115(17) \AA, c=21.108(2) \AA, \beta=97.753(2)^{\circ}, V=$ $5489.6(10) \AA^{3}, Z=2, T=173(2) \mathrm{K}, \mu(\mathrm{MoK} \alpha)=0.535 \mathrm{~mm}^{-1}$, Dcalc $=1.268 \mathrm{~g} / \mathrm{cm}^{3}, 30856$ reflections measured $\left(2.642^{\circ} \leq 2 \Theta \leq 43.932^{\circ}\right), 6710$ unique $\left(R_{\text {int }}=0.0564, R_{\text {sigma }}=0.0451\right)$ which were used in all calculations. The final $R_{1}$ was 0.0811 (I $>2 \sigma(\mathrm{I})$ ) and $w R_{2}$ was 0.1955 (all data).

\section{Refinement model description}

Number of restraints - 967

This report has been created with Olex2, compiled on 2018.05.29 svn.r3508 for OlexSys. 


\section{Compound 3}

Table S10. Crystal data and structure refinement for 3.

Identification code

Empirical formula

Formula weight

Temperature/K

Crystal system

Space group

$\mathrm{a} / \AA$

$\mathrm{b} / \AA$

$\mathrm{c} / \AA$

$\alpha /{ }^{\circ}$

$\beta /{ }^{\circ}$

$\gamma /{ }^{\circ}$

Volume $/ \AA^{3}$

Z

$\rho_{\text {calc }} \mathrm{g} / \mathrm{cm}^{3}$

$\mu / \mathrm{mm}^{-1}$

$\mathrm{F}(000)$

Crystal size $/ \mathrm{mm}^{3}$

Radiation

$2 \Theta$ range for data collection ${ }^{\circ}$

Index ranges

Reflections collected

Independent reflections

Data/restraints/parameters

Goodness-of-fit on $\mathrm{F}^{2}$

Final R indexes $[\mathrm{I}>=2 \sigma(\mathrm{I})]$

Final $\mathrm{R}$ indexes [all data]

Largest diff. peak/hole / e $\AA^{-3}$
3

$\mathrm{C}_{104} \mathrm{H}_{100} \mathrm{Mn}_{4} \mathrm{~N}_{12} \mathrm{O}_{18} \mathrm{Si}_{8}$

2250.43

100(2)

monoclinic

$\mathrm{P} 2{ }_{1} / \mathrm{n}$

15.3244(17)

19.237(2)

19.181(2)

90

107.889(2)

90

$5381.0(11)$

2

1.389

0.618

2328.0

$0.230 \times 0.219 \times 0.104$

$\operatorname{MoK} \alpha(\lambda=0.71073)$

2.992 to 55.9

$-20 \leq \mathrm{h} \leq 19,-25 \leq \mathrm{k} \leq 25,-25 \leq 1 \leq 25$

45344

$12851\left[\mathrm{R}_{\text {int }}=0.0576, \mathrm{R}_{\text {sigma }}=0.0807\right]$

$12851 / 197 / 679$

1.054

$\mathrm{R}_{1}=0.0568, \mathrm{wR}_{2}=0.1508$

$\mathrm{R}_{1}=0.0893, \mathrm{wR}_{2}=0.1698$

$1.19 /-0.73$ 
Table S11. Bond Lengths for 3.

\begin{tabular}{|c|c|c|c|c|c|}
\hline Atom & Atom & Length/Å & Atom & Atom & Length/Å \\
\hline Mn1 & O1 & $2.160(2)$ & C11 & $\mathrm{C} 12$ & $1.391(5)$ \\
\hline Mn1 & $\mathrm{O} 2$ & $2.184(2)$ & $\mathrm{C} 12$ & $\mathrm{C} 13$ & $1.391(6)$ \\
\hline Mn1 & $\mathrm{O} 4^{1}$ & $2.194(2)$ & $\mathrm{C} 13$ & $\mathrm{C} 14$ & $1.367(6)$ \\
\hline Mn1 & $\mathrm{O} 3^{1}$ & $2.204(2)$ & $\mathrm{C} 14$ & $\mathrm{C} 15$ & $1.371(6)$ \\
\hline Mn1 & N1 & $2.295(3)$ & $\mathrm{C} 15$ & $\mathrm{C} 16$ & $1.394(6)$ \\
\hline Mn1 & $\mathrm{N} 2$ & $2.306(3)$ & $\mathrm{C} 17$ & $\mathrm{C} 22$ & $1.386(5)$ \\
\hline Mn1 & $\mathrm{Mn} 2$ & $3.1035(7)$ & $\mathrm{C} 17$ & $\mathrm{C} 18$ & $1.397(5)$ \\
\hline Mn1 & $\mathrm{Mn} 2^{1}$ & $3.1937(7)$ & $\mathrm{C} 18$ & C19 & $1.398(5)$ \\
\hline $\mathrm{Mn} 2$ & $\mathrm{O} 3$ & $2.092(2)$ & $\mathrm{C} 19$ & $\mathrm{C} 20$ & $1.379(6)$ \\
\hline Mn2 & $\mathrm{O} 4^{1}$ & $2.102(2)$ & $\mathrm{C} 20$ & $\mathrm{C} 21$ & $1.365(5)$ \\
\hline $\mathrm{Mn} 2$ & $\mathrm{O} 2$ & $2.117(2)$ & $\mathrm{C} 21$ & $\mathrm{C} 22$ & $1.398(5)$ \\
\hline $\mathrm{Mn} 2$ & $\mathrm{O} 1^{1}$ & $2.131(2)$ & $\mathrm{C} 23$ & $\mathrm{C} 28$ & $1.382(5)$ \\
\hline $\mathrm{Mn} 2$ & N7 & 2.193(3) & $\mathrm{C} 23$ & $\mathrm{C} 24$ & $1.382(5)$ \\
\hline $\mathrm{Mn} 2$ & $\mathrm{Mn} 1^{1}$ & $3.1937(7)$ & $\mathrm{C} 24$ & $\mathrm{C} 25$ & $1.394(5)$ \\
\hline Sil & $\mathrm{O} 1$ & $1.597(2)$ & $\mathrm{C} 25$ & $\mathrm{C} 26$ & $1.370(6)$ \\
\hline Sil & O5 & $1.640(2)$ & $\mathrm{C} 26$ & $\mathrm{C} 27$ & $1.370(7)$ \\
\hline Sil & $\mathrm{O} 8$ & $1.645(2)$ & $\mathrm{C} 27$ & $\mathrm{C} 28$ & $1.399(6)$ \\
\hline Si1 & $\mathrm{C} 11$ & $1.856(3)$ & $\mathrm{C} 29$ & $\mathrm{C} 30$ & $1.389(5)$ \\
\hline $\mathrm{Si} 2$ & $\mathrm{O} 2$ & $1.601(2)$ & $\mathrm{C} 29$ & $\mathrm{C} 34$ & $1.390(5)$ \\
\hline $\mathrm{Si} 2$ & O5 & $1.643(2)$ & $\mathrm{C} 30$ & $\mathrm{C} 31$ & $1.385(5)$ \\
\hline $\mathrm{Si} 2$ & O6 & $1.646(2)$ & $\mathrm{C} 31$ & $\mathrm{C} 32$ & $1.379(7)$ \\
\hline $\mathrm{Si} 2$ & $\mathrm{C} 17$ & $1.861(3)$ & $\mathrm{C} 32$ & $\mathrm{C} 33$ & $1.374(6)$ \\
\hline $\mathrm{Si} 3$ & $\mathrm{O} 3$ & $1.592(2)$ & $\mathrm{C} 33$ & C34 & $1.389(5)$ \\
\hline $\mathrm{Si} 3$ & O7 & $1.643(2)$ & $\mathrm{C} 35$ & C36 & $1.384(5)$ \\
\hline $\mathrm{Si} 3$ & O6 & $1.643(2)$ & $\mathrm{C} 36$ & C37 & $1.382(6)$ \\
\hline $\mathrm{Si} 3$ & $\mathrm{C} 23$ & $1.869(3)$ & $\mathrm{C} 37$ & $\mathrm{C} 38$ & $1.387(6)$ \\
\hline $\mathrm{Si} 4$ & $\mathrm{O} 4$ & $1.593(2)$ & C38 & C39 & $1.377(5)$ \\
\hline Si4 & $\mathrm{O} 8$ & $1.638(2)$ & O9 & $\mathrm{C} 40$ & $1.268(8)$ \\
\hline $\mathrm{Si} 4$ & O7 & $1.644(2)$ & N8 & $\mathrm{C} 43$ & $1.314(9)$ \\
\hline Si4 & $\mathrm{C} 29$ & $1.863(3)$ & N8 & $\mathrm{C} 40$ & $1.320(10)$ \\
\hline $\mathrm{O} 1$ & $\mathrm{Mn} 2^{1}$ & $2.131(2)$ & N8 & $\mathrm{C} 41$ & $1.442(11)$ \\
\hline $\mathrm{O} 3$ & $\mathrm{Mn} 1^{1}$ & $2.204(2)$ & N9 & $\mathrm{C} 53$ & 1.3900 \\
\hline $\mathrm{O} 4$ & $\mathrm{Mn} 2^{1}$ & $2.102(2)$ & N9 & $\mathrm{C} 49$ & 1.3900 \\
\hline $\mathrm{O} 4$ & $\mathrm{Mn} 1^{1}$ & $2.194(2)$ & $\mathrm{C} 53$ & $\mathrm{C} 52$ & 1.3900 \\
\hline N1 & $\mathrm{C} 5$ & $1.334(5)$ & $\mathrm{C} 52$ & $\mathrm{C} 51$ & 1.3900 \\
\hline N1 & $\mathrm{C} 1$ & $1.338(5)$ & C51 & $\mathrm{C} 50$ & 1.3900 \\
\hline N2 & $\mathrm{C} 10$ & $1.331(5)$ & $\mathrm{C} 50$ & C49 & 1.3900 \\
\hline N2 & $\mathrm{C} 6$ & $1.354(5)$ & N9A & $\mathrm{C} 58$ & 1.3900 \\
\hline N7 & $\mathrm{C} 35$ & $1.341(4)$ & N9A & $\mathrm{C} 54$ & 1.3900 \\
\hline
\end{tabular}




\begin{tabular}{|c|c|c|c|c|c|}
\hline Atom & Atom & Length/Å & Atom & Atom & Length/Å \\
\hline N7 & C39 & $1.343(4)$ & C58 & $\mathrm{C} 57$ & 1.3900 \\
\hline $\mathrm{C} 1$ & $\mathrm{C} 2$ & $1.367(5)$ & C57 & C56 & 1.3900 \\
\hline $\mathrm{C} 2$ & $\mathrm{C} 3$ & $1.382(6)$ & C56 & C55 & 1.3900 \\
\hline C3 & $\mathrm{C} 4$ & $1.374(6)$ & C55 & C54 & 1.3900 \\
\hline $\mathrm{C} 4$ & C5 & $1.399(5)$ & N10 & $\mathrm{C} 44$ & 1.3900 \\
\hline C5 & C6 & $1.483(5)$ & N10 & $\mathrm{C} 48$ & 1.3900 \\
\hline C6 & $\mathrm{C} 7$ & $1.397(5)$ & C44 & $\mathrm{C} 45$ & 1.3900 \\
\hline $\mathrm{C} 7$ & $\mathrm{C} 8$ & $1.386(6)$ & $\mathrm{C} 45$ & $\mathrm{C} 46$ & 1.3900 \\
\hline $\mathrm{C} 8$ & C9 & $1.378(6)$ & $\mathrm{C} 46$ & $\mathrm{C} 47$ & 1.3900 \\
\hline C9 & $\mathrm{C} 10$ & $1.397(5)$ & $\mathrm{C} 47$ & $\mathrm{C} 48$ & 1.3900 \\
\hline $\mathrm{C} 11$ & $\mathrm{C} 16$ & $1.385(5)$ & & & \\
\hline
\end{tabular}

${ }^{1} 1-X, 1-Y, 1-Z$

Table S12 Bond Angles for 3.

\begin{tabular}{|c|c|c|c|c|c|c|c|}
\hline Atom & Atom & Atom & Angle/ ${ }^{\circ}$ & Atom & Atom & Atom & Angle $/^{\circ}$ \\
\hline $\mathrm{O} 1$ & Mn1 & $\mathrm{O} 2$ & $86.45(8)$ & $\mathrm{Si3}$ & O6 & $\mathrm{Si} 2$ & $131.36(14)$ \\
\hline $\mathrm{O} 1$ & Mn1 & $\mathrm{O} 4^{1}$ & $132.26(8)$ & $\mathrm{Si} 3$ & $\mathrm{O} 7$ & $\mathrm{Si} 4$ & $129.26(13)$ \\
\hline $\mathrm{O} 2$ & Mn1 & $\mathrm{O} 4^{1}$ & $76.62(8)$ & $\mathrm{Si} 4$ & O8 & Sil & $131.37(14)$ \\
\hline $\mathrm{O} 1$ & Mn1 & $\mathrm{O} 3^{1}$ & $74.97(8)$ & $\mathrm{C} 5$ & N1 & $\mathrm{C} 1$ & $118.7(3)$ \\
\hline $\mathrm{O} 2$ & Mn1 & $\mathrm{O} 3^{1}$ & $133.15(8)$ & $\mathrm{C} 5$ & N1 & Mn1 & $119.5(3)$ \\
\hline $\mathrm{O} 4^{1}$ & Mn1 & $\mathrm{O} 3^{1}$ & $84.81(8)$ & $\mathrm{C} 1$ & N1 & Mn1 & $120.6(2)$ \\
\hline $\mathrm{O} 1$ & Mn1 & N1 & $88.65(10)$ & $\mathrm{C} 10$ & N2 & C6 & $119.7(3)$ \\
\hline $\mathrm{O} 2$ & Mn1 & N1 & $137.21(10)$ & $\mathrm{C} 10$ & $\mathrm{~N} 2$ & Mn1 & $121.3(3)$ \\
\hline $\mathrm{O} 4^{1}$ & Mn1 & N1 & $133.01(10)$ & C6 & $\mathrm{N} 2$ & Mn1 & $118.2(2)$ \\
\hline $\mathrm{O} 3^{1}$ & Mn1 & N1 & $85.76(10)$ & $\mathrm{C} 35$ & N7 & C39 & $117.8(3)$ \\
\hline $\mathrm{O} 1$ & Mn1 & $\mathrm{N} 2$ & $140.27(10)$ & $\mathrm{C} 35$ & N7 & $\mathrm{Mn} 2$ & $119.5(2)$ \\
\hline $\mathrm{O} 2$ & Mn1 & N2 & $88.31(10)$ & C39 & N7 & $\mathrm{Mn} 2$ & $120.2(2)$ \\
\hline $\mathrm{O} 4^{1}$ & Mn1 & $\mathrm{N} 2$ & $84.15(9)$ & N1 & $\mathrm{C} 1$ & $\mathrm{C} 2$ & $123.0(4)$ \\
\hline $\mathrm{O} 3^{1}$ & Mn1 & N2 & $132.46(10)$ & $\mathrm{C} 1$ & $\mathrm{C} 2$ & C3 & $118.6(4)$ \\
\hline N1 & Mn1 & N2 & $69.29(11)$ & $\mathrm{C} 4$ & $\mathrm{C} 3$ & $\mathrm{C} 2$ & $119.4(4)$ \\
\hline $\mathrm{O} 1$ & Mn1 & Mn2 & $96.71(6)$ & C3 & $\mathrm{C} 4$ & C5 & $118.6(4)$ \\
\hline $\mathrm{O} 2$ & Mn1 & Mn2 & $42.95(6)$ & N1 & $\mathrm{C} 5$ & $\mathrm{C} 4$ & $121.7(4)$ \\
\hline $\mathrm{O} 4^{1}$ & Mn1 & $\mathrm{Mn} 2$ & $42.59(6)$ & N1 & C5 & C6 & $114.4(3)$ \\
\hline $\mathrm{O} 3^{1}$ & Mn1 & $\mathrm{Mn} 2$ & $96.33(6)$ & $\mathrm{C} 4$ & $\mathrm{C} 5$ & C6 & $123.8(3)$ \\
\hline N1 & Mn1 & $\operatorname{Mn} 2$ & $174.59(8)$ & $\mathrm{N} 2$ & C6 & $\mathrm{C} 7$ & $121.3(4)$ \\
\hline N2 & Mn1 & Mn2 & $105.83(8)$ & $\mathrm{N} 2$ & C6 & $\mathrm{C} 5$ & $115.4(3)$ \\
\hline $\mathrm{O} 1$ & Mn1 & $\mathrm{Mn} 2^{1}$ & $41.57(6)$ & $\mathrm{C} 7$ & C6 & $\mathrm{C} 5$ & $123.3(4)$ \\
\hline $\mathrm{O} 2$ & Mn1 & $\mathrm{Mn} 2^{1}$ & $99.31(6)$ & $\mathrm{C} 8$ & $\mathrm{C} 7$ & C6 & $118.5(4)$ \\
\hline $\mathrm{O} 4^{1}$ & Mn1 & $\mathrm{Mn} 2^{1}$ & $97.30(6)$ & C9 & $\mathrm{C} 8$ & $\mathrm{C} 7$ & $120.0(4)$ \\
\hline $\mathrm{O} 3^{1}$ & Mn1 & $\operatorname{Mn} 2^{1}$ & $40.66(5)$ & $\mathrm{C} 8$ & C9 & $\mathrm{C} 10$ & $118.6(4)$ \\
\hline
\end{tabular}




\begin{tabular}{|c|c|c|c|c|c|c|c|}
\hline Atom & Atom & Atom & Angle ${ }^{\circ}$ & Atom & Atom & Atom & Angle $/^{\circ}$ \\
\hline N1 & Mn1 & $\mathrm{Mn} 2^{1}$ & $104.74(8)$ & N2 & $\mathrm{C} 10$ & C9 & $121.9(4)$ \\
\hline $\mathrm{N} 2$ & Mn1 & $\mathrm{Mn} 2^{1}$ & $172.38(8)$ & $\mathrm{C} 16$ & $\mathrm{C} 11$ & $\mathrm{C} 12$ & $116.5(3)$ \\
\hline $\mathrm{Mn} 2$ & Mn1 & $\mathrm{Mn} 2^{1}$ & $79.930(17)$ & $\mathrm{C} 16$ & $\mathrm{C} 11$ & Sil & $124.0(3)$ \\
\hline $\mathrm{O} 3$ & $\mathrm{Mn} 2$ & $\mathrm{O} 4^{1}$ & $150.14(8)$ & $\mathrm{C} 12$ & $\mathrm{C} 11$ & Sil & $119.5(3)$ \\
\hline $\mathrm{O} 3$ & $\mathrm{Mn} 2$ & $\mathrm{O} 2$ & $94.20(8)$ & $\mathrm{C} 13$ & $\mathrm{C} 12$ & $\mathrm{C} 11$ & $122.2(4)$ \\
\hline $\mathrm{O} 4^{1}$ & $\mathrm{Mn} 2$ & $\mathrm{O} 2$ & $80.09(8)$ & $\mathrm{C} 14$ & $\mathrm{C} 13$ & $\mathrm{C} 12$ & $120.1(4)$ \\
\hline $\mathrm{O} 3$ & $\mathrm{Mn} 2$ & $\mathrm{O} 1^{1}$ & $77.91(8)$ & $\mathrm{C} 13$ & $\mathrm{C} 14$ & $\mathrm{C} 15$ & $119.1(4)$ \\
\hline $\mathrm{O} 4^{1}$ & $\mathrm{Mn} 2$ & $\mathrm{O} 1^{1}$ & $92.81(8)$ & $\mathrm{C} 14$ & $\mathrm{C} 15$ & $\mathrm{C} 16$ & $120.8(4)$ \\
\hline $\mathrm{O} 2$ & $\mathrm{Mn} 2$ & $\mathrm{O} 1^{1}$ & $150.79(8)$ & $\mathrm{C} 11$ & $\mathrm{C} 16$ & $\mathrm{C} 15$ & $121.3(4)$ \\
\hline $\mathrm{O} 3$ & $\mathrm{Mn} 2$ & N7 & $105.63(9)$ & $\mathrm{C} 22$ & $\mathrm{C} 17$ & $\mathrm{C} 18$ & $117.0(3)$ \\
\hline $\mathrm{O}^{1}$ & $\mathrm{Mn} 2$ & N7 & $103.75(10)$ & $\mathrm{C} 22$ & $\mathrm{C} 17$ & $\mathrm{Si} 2$ & $122.3(3)$ \\
\hline $\mathrm{O} 2$ & $\mathrm{Mn} 2$ & N7 & 110.99(9) & $\mathrm{C} 18$ & $\mathrm{C} 17$ & $\mathrm{Si} 2$ & $120.7(3)$ \\
\hline $\mathrm{O} 1^{1}$ & $\mathrm{Mn} 2$ & N7 & $98.22(9)$ & $\mathrm{C} 17$ & $\mathrm{C} 18$ & C19 & $121.4(4)$ \\
\hline $\mathrm{O} 3$ & $\mathrm{Mn} 2$ & Mn1 & $112.52(6)$ & $\mathrm{C} 20$ & C19 & $\mathrm{C} 18$ & $120.1(4)$ \\
\hline $\mathrm{O} 4^{1}$ & Mn2 & Mn1 & $44.94(6)$ & $\mathrm{C} 21$ & $\mathrm{C} 20$ & C19 & $119.5(3)$ \\
\hline $\mathrm{O} 2$ & Mn2 & Mn1 & $44.67(6)$ & $\mathrm{C} 20$ & $\mathrm{C} 21$ & $\mathrm{C} 22$ & $120.5(4)$ \\
\hline $\mathrm{O} 1^{1}$ & $\mathrm{Mn} 2$ & Mn1 & $112.36(6)$ & $\mathrm{C} 17$ & $\mathrm{C} 22$ & $\mathrm{C} 21$ & $121.6(3)$ \\
\hline N7 & $\mathrm{Mn} 2$ & Mn1 & $134.84(8)$ & $\mathrm{C} 28$ & $\mathrm{C} 23$ & $\mathrm{C} 24$ & $117.2(3)$ \\
\hline $\mathrm{O} 3$ & $\mathrm{Mn} 2$ & $\mathrm{Mn}^{1}$ & $43.34(6)$ & $\mathrm{C} 28$ & $\mathrm{C} 23$ & $\mathrm{Si} 3$ & $122.2(3)$ \\
\hline $\mathrm{O} 4^{1}$ & $\mathrm{Mn} 2$ & Mn1 ${ }^{1}$ & $113.00(6)$ & $\mathrm{C} 24$ & $\mathrm{C} 23$ & $\mathrm{Si} 3$ & $120.3(3)$ \\
\hline $\mathrm{O} 2$ & $\mathrm{Mn} 2$ & $\mathrm{Mn}^{1}{ }^{1}$ & $115.05(6)$ & $\mathrm{C} 23$ & $\mathrm{C} 24$ & $\mathrm{C} 25$ & $122.1(4)$ \\
\hline $\mathrm{O} 1^{1}$ & $\mathrm{Mn} 2$ & $\operatorname{Mn} 1^{1}$ & $42.25(6)$ & $\mathrm{C} 26$ & $\mathrm{C} 25$ & $\mathrm{C} 24$ & $119.9(4)$ \\
\hline N7 & $\mathrm{Mn} 2$ & $\mathrm{Mn}^{1}{ }^{1}$ & $124.50(7)$ & $\mathrm{C} 25$ & $\mathrm{C} 26$ & $\mathrm{C} 27$ & $119.1(4)$ \\
\hline Mn1 & $\mathrm{Mn} 2$ & $\mathrm{Mn}^{1}{ }^{1}$ & $100.069(17)$ & $\mathrm{C} 26$ & $\mathrm{C} 27$ & $\mathrm{C} 28$ & $120.9(4)$ \\
\hline $\mathrm{O} 1$ & Sil & $\mathrm{O} 5$ & $110.79(12)$ & $\mathrm{C} 23$ & $\mathrm{C} 28$ & $\mathrm{C} 27$ & $120.8(4)$ \\
\hline $\mathrm{O} 1$ & Sil & O8 & $109.90(12)$ & C30 & C29 & C34 & 117.1(3) \\
\hline $\mathrm{O} 5$ & Sil & O8 & $108.45(12)$ & C30 & C29 & $\mathrm{Si} 4$ & $122.4(3)$ \\
\hline O1 & Sil & $\mathrm{C} 11$ & $113.11(13)$ & C34 & $\mathrm{C} 29$ & $\mathrm{Si} 4$ & $120.6(3)$ \\
\hline $\mathrm{O} 5$ & Sil & $\mathrm{C} 11$ & $107.71(14)$ & C31 & C30 & $\mathrm{C} 29$ & $121.2(4)$ \\
\hline O8 & Si1 & $\mathrm{C} 11$ & $106.70(13)$ & C32 & C31 & $\mathrm{C} 30$ & $120.5(4)$ \\
\hline $\mathrm{O} 2$ & $\mathrm{Si} 2$ & $\mathrm{O} 5$ & $110.81(12)$ & C33 & C32 & C31 & $119.6(4)$ \\
\hline $\mathrm{O} 2$ & $\mathrm{Si} 2$ & O6 & $110.51(12)$ & C32 & C33 & C34 & $119.5(4)$ \\
\hline O5 & $\mathrm{Si} 2$ & O6 & $108.97(12)$ & C33 & C34 & C29 & $122.1(4)$ \\
\hline $\mathrm{O} 2$ & $\mathrm{Si} 2$ & $\mathrm{C} 17$ & $115.20(13)$ & N7 & $\mathrm{C} 35$ & $\mathrm{C} 36$ & $123.3(4)$ \\
\hline O5 & $\mathrm{Si} 2$ & C17 & $105.37(13)$ & C37 & C36 & C35 & $118.2(4)$ \\
\hline O6 & $\mathrm{Si} 2$ & $\mathrm{C} 17$ & $105.65(13)$ & C36 & C37 & $\mathrm{C} 38$ & $118.9(4)$ \\
\hline $\mathrm{O} 3$ & $\mathrm{Si} 3$ & $\mathrm{O} 7$ & $109.71(12)$ & C39 & C38 & C37 & $119.3(4)$ \\
\hline $\mathrm{O} 3$ & $\mathrm{Si} 3$ & O6 & $111.13(11)$ & N7 & C39 & C38 & $122.4(3)$ \\
\hline O7 & $\mathrm{Si} 3$ & O6 & $108.99(12)$ & $\mathrm{C} 43$ & N8 & $\mathrm{C} 40$ & $124.3(9)$ \\
\hline $\mathrm{O} 3$ & $\mathrm{Si} 3$ & $\mathrm{C} 23$ & $111.99(13)$ & $\mathrm{C} 43$ & N8 & $\mathrm{C} 41$ & $121.4(10)$ \\
\hline $\mathrm{O} 7$ & $\mathrm{Si} 3$ & $\mathrm{C} 23$ & $107.03(13)$ & $\mathrm{C} 40$ & N8 & C41 & 114.2(8) \\
\hline
\end{tabular}




\begin{tabular}{|c|c|c|c|c|c|c|c|}
\hline Atom & Atom & Atom & Angle $/^{\circ}$ & Atom & Atom & Atom & Angle ${ }^{\circ}$ \\
\hline O6 & $\mathrm{Si3}$ & $\mathrm{C} 23$ & $107.87(14)$ & O9 & $\mathrm{C} 40$ & N8 & $110.9(8)$ \\
\hline $\mathrm{O} 4$ & $\mathrm{Si} 4$ & $\mathrm{O} 8$ & $111.78(12)$ & C53 & N9 & C49 & 120.0 \\
\hline $\mathrm{O} 4$ & $\mathrm{Si} 4$ & $\mathrm{O} 7$ & $110.23(12)$ & N9 & C53 & C52 & 120.0 \\
\hline $\mathrm{O} 8$ & $\mathrm{Si} 4$ & $\mathrm{O} 7$ & $108.80(12)$ & C53 & C52 & C51 & 120.0 \\
\hline $\mathrm{O} 4$ & $\mathrm{Si} 4$ & $\mathrm{C} 29$ & $112.69(14)$ & C52 & C51 & C50 & 120.0 \\
\hline $\mathrm{O} 8$ & $\mathrm{Si} 4$ & $\mathrm{C} 29$ & $106.15(14)$ & C49 & $\mathrm{C} 50$ & C51 & 120.0 \\
\hline $\mathrm{O} 7$ & $\mathrm{Si} 4$ & $\mathrm{C} 29$ & $106.95(13)$ & $\mathrm{C} 50$ & C49 & N9 & 120.0 \\
\hline Sil & O1 & $\operatorname{Mn} 2^{1}$ & $128.83(12)$ & C58 & N9A & C54 & 120.0 \\
\hline Sil & $\mathrm{O} 1$ & Mn1 & $128.04(12)$ & N9A & C58 & C57 & 120.0 \\
\hline $\mathrm{Mn} 2^{1}$ & $\mathrm{O} 1$ & Mn1 & 96.19(9) & $\mathrm{C} 56$ & C57 & C58 & 120.0 \\
\hline $\mathrm{Si} 2$ & $\mathrm{O} 2$ & $\operatorname{Mn} 2$ & $127.73(12)$ & C55 & C56 & C57 & 120.0 \\
\hline $\mathrm{Si} 2$ & $\mathrm{O} 2$ & Mn1 & $127.99(12)$ & C56 & C55 & C54 & 120.0 \\
\hline Mn2 & $\mathrm{O} 2$ & Mn1 & $92.38(8)$ & C55 & C54 & N9A & 120.0 \\
\hline $\mathrm{Si} 3$ & $\mathrm{O} 3$ & $\mathrm{Mn} 2$ & $127.92(12)$ & C44 & N10 & $\mathrm{C} 48$ & 120.0 \\
\hline $\mathrm{Si} 3$ & $\mathrm{O} 3$ & $\mathrm{Mn}^{1}{ }^{1}$ & $128.90(12)$ & N10 & C44 & $\mathrm{C} 45$ & 120.0 \\
\hline $\mathrm{Mn} 2$ & $\mathrm{O} 3$ & $\mathrm{Mn}^{1}{ }^{1}$ & $96.00(8)$ & $\mathrm{C} 46$ & $\mathrm{C} 45$ & C44 & 120.0 \\
\hline $\mathrm{Si} 4$ & $\mathrm{O} 4$ & $\operatorname{Mn} 2^{1}$ & $128.06(13)$ & $\mathrm{C} 45$ & C46 & C47 & 120.0 \\
\hline $\mathrm{Si} 4$ & O4 & $\mathrm{Mn}^{1}{ }^{1}$ & $129.09(12)$ & $\mathrm{C} 46$ & C47 & C48 & 120.0 \\
\hline $\mathrm{Mn} 2^{1}$ & $\mathrm{O} 4$ & $\mathrm{Mn}^{1}{ }^{1}$ & $92.47(8)$ & $\mathrm{C} 47$ & $\mathrm{C} 48$ & N10 & 120.0 \\
\hline Sil & $\mathrm{O} 5$ & $\mathrm{Si} 2$ & $129.03(14)$ & & & & \\
\hline
\end{tabular}

${ }^{1} 1-X, 1-Y, 1-Z$

\section{Experimental}

A single crystals of $\mathrm{C}_{104} \mathrm{H}_{100} \mathrm{Mn}_{4} \mathrm{~N}_{12} \mathrm{O}_{18} \mathrm{Si}_{8}$ was selected and mounted on a diffractometer. The crystal was kept at 100(2) K during data collection. Using Olex $2^{4}$ as a GUI, the structure was solved with the ShelXS structure solution program using Structure Expansion and refined with the $\mathrm{XL}^{2}$ refinement package using Least Squares minimisation.

\section{Crystal structure determination}

Crystal Data for $\mathrm{C}_{104} \mathrm{H}_{100} \mathrm{Mn}_{4} \mathrm{~N}_{12} \mathrm{O}_{18} \mathrm{Si}_{8}(M=2250.43 \mathrm{~g} / \mathrm{mol})$ : monoclinic, space group $\mathrm{P} 2{ }_{1} / \mathrm{n}$ (no. 14), $a=15.3244(17) \AA, b=19.237(2) \AA, c=19.181(2) \AA, \beta=107.889(2)^{\circ}, V=$ $5381.0(11) \AA^{3}, Z=2, T=100(2) \mathrm{K}, \mu(\mathrm{MoK} \alpha)=0.618 \mathrm{~mm}^{-1}$, Dcalc $=1.389 \mathrm{~g} / \mathrm{cm}^{3}, 45344$ reflections measured $\left(2.992^{\circ} \leq 2 \Theta \leq 55.9^{\circ}\right), 12851$ unique $\left(R_{\text {int }}=0.0576, R_{\text {sigma }}=0.0807\right)$ which were used in all calculations. The final $R_{1}$ was $0.0568(\mathrm{I}>2 \sigma(\mathrm{I}))$ and $w R_{2}$ was 0.1698 (all data).

\section{Refinement model description}

Number of restraints - 197 


\section{Compound 4}

Table S13 Crystal data and structure refinement for 4.

Identification code

Empirical formula

Formula weight

Temperature/K

Crystal system

Space group

$\mathrm{a} / \AA$

$\mathrm{b} / \AA$

$\mathrm{c} / \AA$

$\alpha /{ }^{\circ}$

$\beta /{ }^{\circ}$

$\gamma / 0$

Volume $/ \AA^{3}$

Z

$\rho_{\text {calc }} \mathrm{g} / \mathrm{cm}^{3}$

$\mu / \mathrm{mm}^{-1}$

$\mathrm{F}(000)$

Crystal size $/ \mathrm{mm}^{3}$

Radiation

$2 \Theta$ range for data collection ${ }^{\circ}$

Index ranges

Reflections collected

Independent reflections

Data/restraints/parameters

Goodness-of-fit on $\mathrm{F}^{2}$

Final R indexes [I $>=2 \sigma(\mathrm{I})]$

Final R indexes [all data]

Largest diff. peak/hole / e $\AA^{-3}$
4

$\mathrm{C}_{107} \mathrm{H}_{117} \mathrm{Li}_{2} \mathrm{Mn}_{3} \mathrm{~N}_{7} \mathrm{O}_{31} \mathrm{Si}_{14}$

2569.03

100.02

triclinic

P-1

15.499(3)

19.247(3)

$25.649(5)$

$100.410(3)$

$92.708(3)$

97.495(3)

7441(2)

2

1.147

0.426

2666.0

$0.686 \times 0.392 \times 0.274$

$\operatorname{MoK} \alpha(\lambda=0.71073)$

2.944 to 56.064

$-17 \leq \mathrm{h} \leq 20,-25 \leq \mathrm{k} \leq 25,-33 \leq 1 \leq 33$

111228

$34404\left[\mathrm{R}_{\text {int }}=0.0409, \mathrm{R}_{\text {sigma }}=0.0514\right]$

$34404 / 1360 / 1705$

1.035

$\mathrm{R}_{1}=0.0841, \mathrm{wR}_{2}=0.1995$

$\mathrm{R}_{1}=0.1162, \mathrm{wR}_{2}=0.2268$

$1.05 /-0.73$

Table S14 Bond Lengths for 4.

\begin{tabular}{|c|c|c|c|c|c|}
\hline Atom & Atom & Length/Å & Atom & Atom & Length/Å \\
\hline Mn1 & Mn2 & $3.2112(9)$ & $\mathrm{C} 7$ & $\mathrm{C} 8$ & $1.395(7)$ \\
\hline Mn1 & $\mathrm{O} 1$ & $2.085(3)$ & $\mathrm{C} 7$ & $\mathrm{C} 12$ & $1.405(8)$ \\
\hline Mn1 & $\mathrm{O} 2$ & $2.089(3)$ & $\mathrm{C} 8$ & C9 & $1.380(9)$ \\
\hline Mn1 & $\mathrm{O} 4$ & $2.082(3)$ & C9 & $\mathrm{C} 10$ & $1.357(11)$ \\
\hline Mn1 & $\mathrm{O} 15$ & $2.197(3)$ & $\mathrm{C} 10$ & C11 & $1.371(9)$ \\
\hline Mn1 & $\mathrm{O} 27$ & $2.249(3)$ & $\mathrm{C} 11$ & $\mathrm{C} 12$ & $1.386(8)$ \\
\hline Mn1 & Li1 & $2.972(8)$ & $\mathrm{C} 13$ & $\mathrm{C} 18$ & 1.3900 \\
\hline Mn1 & $\mathrm{Li} 2$ & $2.877(7)$ & $\mathrm{C} 13$ & $\mathrm{C} 14$ & 1.3900 \\
\hline $\mathrm{Mn} 2$ & Mn3 & $3.2103(9)$ & $\mathrm{C} 18$ & $\mathrm{C} 17$ & 1.3900 \\
\hline
\end{tabular}




\begin{tabular}{|c|c|c|c|c|c|}
\hline Atom & Atom & Length/Å & Atom & Atom & Length/Å \\
\hline Mn2 & $\mathrm{O} 3$ & $2.054(3)$ & $\mathrm{C} 17$ & C16 & 1.3900 \\
\hline $\mathrm{Mn} 2$ & $\mathrm{O} 4$ & $2.192(3)$ & $\mathrm{C} 16$ & $\mathrm{C} 15$ & 1.3900 \\
\hline $\mathrm{Mn} 2$ & $\mathrm{O} 14$ & $2.078(3)$ & $\mathrm{C} 15$ & $\mathrm{C} 14$ & 1.3900 \\
\hline $\mathrm{Mn} 2$ & $\mathrm{O} 15$ & $2.139(3)$ & C13A & $\mathrm{C} 14 \mathrm{~A}$ & 1.3900 \\
\hline $\mathrm{Mn} 2$ & O16 & $2.193(3)$ & C13A & C18A & 1.3900 \\
\hline $\mathrm{Mn} 2$ & $\mathrm{Li} 2$ & $2.883(7)$ & C14A & $\mathrm{C} 15 \mathrm{~A}$ & 1.3900 \\
\hline Mn3 & $\mathrm{O} 14$ & $2.160(3)$ & $\mathrm{C} 15 \mathrm{~A}$ & C16A & 1.3900 \\
\hline Mn3 & $\mathrm{O} 16$ & $2.108(3)$ & C16A & C17A & 1.3900 \\
\hline Mn3 & $\mathrm{O} 17$ & $1.993(3)$ & C17A & C18A & 1.3900 \\
\hline Mn3 & $\mathrm{O} 26$ & $2.167(4)$ & C19 & $\mathrm{C} 20$ & $1.377(10)$ \\
\hline Mn3 & N1 & $2.310(4)$ & C19 & $\mathrm{C} 24$ & $1.383(9)$ \\
\hline Sil & $\mathrm{O} 1$ & $1.577(3)$ & $\mathrm{C} 20$ & $\mathrm{C} 21$ & $1.390(10)$ \\
\hline Sil & O9 & $1.651(3)$ & $\mathrm{C} 21$ & $\mathrm{C} 22$ & $1.372(14)$ \\
\hline Sil & $\mathrm{O} 12$ & $1.644(3)$ & $\mathrm{C} 22$ & $\mathrm{C} 23$ & $1.314(13)$ \\
\hline Sil & $\mathrm{C} 1$ & $1.866(5)$ & $\mathrm{C} 23$ & $\mathrm{C} 24$ & $1.405(9)$ \\
\hline Sil & $\mathrm{Li} 2$ & $3.169(7)$ & $\mathrm{C} 25$ & $\mathrm{C} 26$ & $1.398(8)$ \\
\hline $\mathrm{Si} 2$ & O6 & $1.609(3)$ & $\mathrm{C} 25$ & $\mathrm{C} 30$ & $1.385(8)$ \\
\hline $\mathrm{Si} 2$ & O8 & $1.630(4)$ & $\mathrm{C} 26$ & $\mathrm{C} 27$ & $1.387(10)$ \\
\hline $\mathrm{Si} 2$ & O9 & $1.621(3)$ & $\mathrm{C} 27$ & $\mathrm{C} 28$ & $1.363(13)$ \\
\hline $\mathrm{Si} 2$ & $\mathrm{C} 7$ & $1.853(5)$ & $\mathrm{C} 28$ & $\mathrm{C} 29$ & $1.345(13)$ \\
\hline $\mathrm{Si} 3$ & $\mathrm{O} 7$ & $1.613(4)$ & $\mathrm{C} 29$ & C30 & $1.416(9)$ \\
\hline $\mathrm{Si} 3$ & O8 & $1.614(4)$ & C31 & C32 & 1.3900 \\
\hline $\mathrm{Si} 3$ & $\mathrm{O} 10$ & $1.603(4)$ & C31 & $\mathrm{C} 36$ & 1.3900 \\
\hline $\mathrm{Si} 3$ & C13 & $1.836(7)$ & C32 & C33 & 1.3900 \\
\hline $\mathrm{Si} 3$ & C13A & $1.878(16)$ & C33 & C34 & 1.3900 \\
\hline $\mathrm{Si} 4$ & $\mathrm{O} 2$ & $1.577(3)$ & C34 & C35 & 1.3900 \\
\hline $\mathrm{Si} 4$ & $\mathrm{O} 10$ & $1.642(4)$ & C35 & C36 & 1.3900 \\
\hline $\mathrm{Si} 4$ & $\mathrm{O} 12$ & $1.629(4)$ & C31A & C36A & 1.3900 \\
\hline $\mathrm{Si} 4$ & C19 & $1.871(5)$ & C31A & C32A & 1.3900 \\
\hline $\mathrm{Si} 4$ & Li1 & $3.132(9)$ & C36A & C35A & 1.3900 \\
\hline $\mathrm{Si} 5$ & $\mathrm{O} 3$ & $1.576(3)$ & C35A & C34A & 1.3900 \\
\hline $\mathrm{Si} 5$ & O5 & $1.643(4)$ & C34A & C33A & 1.3900 \\
\hline $\mathrm{Si} 5$ & O6 & $1.627(3)$ & C33A & C32A & 1.3900 \\
\hline $\mathrm{Si} 5$ & $\mathrm{C} 25$ & $1.868(5)$ & C37 & C38 & 1.3900 \\
\hline $\mathrm{Si} 6$ & O5 & $1.617(4)$ & C37 & $\mathrm{C} 42$ & 1.3900 \\
\hline $\mathrm{Si} 6$ & $\mathrm{O} 7$ & $1.591(4)$ & C38 & C39 & 1.3900 \\
\hline $\mathrm{Si} 6$ & O11 & $1.605(4)$ & C39 & $\mathrm{C} 40$ & 1.3900 \\
\hline $\mathrm{Si} 6$ & C31 & $1.847(5)$ & $\mathrm{C} 40$ & $\mathrm{C} 41$ & 1.3900 \\
\hline $\mathrm{Si} 6$ & C31A & $1.920(18)$ & C41 & $\mathrm{C} 42$ & 1.3900 \\
\hline $\mathrm{Si} 7$ & O4 & $1.594(3)$ & $\mathrm{C} 43$ & $\mathrm{C} 44$ & $1.402(8)$ \\
\hline Si7 & O11 & $1.625(4)$ & $\mathrm{C} 43$ & $\mathrm{C} 48$ & $1.368(7)$ \\
\hline
\end{tabular}




\begin{tabular}{|c|c|c|c|c|c|}
\hline Atom & Atom & Length/Å & Atom & Atom & Length/Å \\
\hline $\mathrm{Si7}$ & $\mathrm{O} 13$ & $1.615(4)$ & $\mathrm{C} 44$ & $\mathrm{C} 45$ & $1.402(8)$ \\
\hline $\mathrm{Si} 7$ & C37 & $1.853(14)$ & $\mathrm{C} 45$ & $\mathrm{C} 46$ & $1.382(10)$ \\
\hline $\mathrm{Si} 7$ & $\mathrm{C} 42 \mathrm{~A}$ & $1.883(4)$ & $\mathrm{C} 46$ & $\mathrm{C} 47$ & $1.341(9)$ \\
\hline Si8 & O17 & $1.570(4)$ & $\mathrm{C} 47$ & C48 & $1.407(7)$ \\
\hline $\mathrm{Si} 8$ & O19 & $1.645(4)$ & C49 & $\mathrm{C} 50$ & 1.3900 \\
\hline $\mathrm{Si} 8$ & $\mathrm{O} 20$ & $1.647(3)$ & C49 & C54 & 1.3900 \\
\hline $\mathrm{Si} 8$ & $\mathrm{C} 43$ & $1.865(4)$ & C50 & C51 & 1.3900 \\
\hline $\mathrm{Si} 9$ & O18 & $1.595(4)$ & C51 & C52 & 1.3900 \\
\hline $\mathrm{Si} 9$ & O19 & $1.616(3)$ & $\mathrm{C} 52$ & C53 & 1.3900 \\
\hline $\mathrm{Si} 9$ & $\mathrm{O} 22$ & $1.638(3)$ & $\mathrm{C} 53$ & C54 & 1.3900 \\
\hline $\mathrm{Si} 9$ & $\mathrm{C} 49$ & $1.92(3)$ & $\mathrm{C} 49 \mathrm{~A}$ & $\mathrm{C} 50 \mathrm{~A}$ & 1.3900 \\
\hline $\mathrm{Si} 9$ & C49A & $1.863(9)$ & C49A & C54A & 1.3900 \\
\hline Sil0 & $\mathrm{O} 20$ & $1.611(3)$ & $\mathrm{C} 50 \mathrm{~A}$ & C51A & 1.3900 \\
\hline Sil0 & $\mathrm{O} 21$ & $1.624(3)$ & $\mathrm{C} 51 \mathrm{~A}$ & C52A & 1.3900 \\
\hline Sil0 & $\mathrm{O} 23$ & $1.616(3)$ & $\mathrm{C} 52 \mathrm{~A}$ & C53A & 1.3900 \\
\hline Sil0 & C55 & $1.873(5)$ & $\mathrm{C} 53 \mathrm{~A}$ & C54A & 1.3900 \\
\hline Sil0 & C55A & $1.884(11)$ & C55 & C56 & 1.3900 \\
\hline Sil1 & $\mathrm{O} 21$ & $1.616(3)$ & $\mathrm{C} 55$ & C60 & 1.3900 \\
\hline Sil1 & $\mathrm{O} 22$ & $1.622(3)$ & $\mathrm{C} 56$ & C57 & 1.3900 \\
\hline Sil1 & $\mathrm{O} 24$ & $1.611(3)$ & $\mathrm{C} 57$ & C58 & 1.3900 \\
\hline Sil1 & C61 & $1.852(7)$ & C58 & C59 & 1.3900 \\
\hline Si11 & C61A & $1.931(17)$ & C59 & C60 & 1.3900 \\
\hline Si12 & O16 & $1.596(3)$ & $\mathrm{C} 55 \mathrm{~A}$ & C56A & 1.3900 \\
\hline Si12 & $\mathrm{O} 23$ & $1.653(3)$ & $\mathrm{C} 55 \mathrm{~A}$ & C60A & 1.3900 \\
\hline Si12 & $\mathrm{O} 25$ & $1.639(3)$ & C56A & C57A & 1.3900 \\
\hline Si12 & C67 & $1.859(5)$ & C57A & C58A & 1.3900 \\
\hline Si13 & O15 & $1.600(3)$ & C58A & C59A & 1.3900 \\
\hline Si13 & $\mathrm{O} 24$ & $1.644(3)$ & C59A & C60A & 1.3900 \\
\hline Si13 & $\mathrm{O} 25$ & $1.627(3)$ & C61 & C62 & 1.3900 \\
\hline Si13 & C73 & $1.868(4)$ & C61 & C66 & 1.3900 \\
\hline Sil4 & O13 & $1.631(4)$ & C62 & C63 & 1.3900 \\
\hline Sil4 & O14 & $1.595(3)$ & C63 & C64 & 1.3900 \\
\hline Si14 & O18 & $1.629(4)$ & C64 & C65 & 1.3900 \\
\hline Si14 & C79 & $1.858(6)$ & C65 & C66 & 1.3900 \\
\hline $\mathrm{O} 1$ & $\mathrm{Li} 2$ & $1.885(8)$ & C61A & C62A & 1.3900 \\
\hline $\mathrm{O} 2$ & Li1 & $1.907(9)$ & C61A & C66A & 1.3900 \\
\hline $\mathrm{O} 3$ & $\mathrm{Li} 2$ & $1.995(8)$ & C62A & C63A & 1.3900 \\
\hline $\mathrm{O} 15$ & $\mathrm{Li} 2$ & $2.050(8)$ & C63A & C64A & 1.3900 \\
\hline $\mathrm{O} 26$ & $\mathrm{C} 90$ & $1.248(6)$ & C64A & C65A & 1.3900 \\
\hline $\mathrm{O} 27$ & C96 & $1.248(5)$ & C65A & C66A & 1.3900 \\
\hline $\mathrm{O} 27$ & Li1 & $2.027(9)$ & C67 & C68 & $1.392(7)$ \\
\hline
\end{tabular}




\begin{tabular}{|c|c|c|c|c|c|}
\hline Atom & Atom & Length/Å & Atom & Atom & Length/Å \\
\hline $\mathrm{O} 28$ & $\mathrm{C} 102$ & $1.231(8)$ & C67 & C72 & $1.385(8)$ \\
\hline $\mathrm{O} 28$ & Li1 & $1.875(10)$ & $\mathrm{C} 68$ & C69 & $1.433(9)$ \\
\hline O29 & C99 & $1.167(8)$ & C69 & $\mathrm{C} 70$ & $1.365(11)$ \\
\hline O29 & Li1 & $1.890(9)$ & $\mathrm{C} 70$ & C71 & $1.303(11)$ \\
\hline $\mathrm{O} 30$ & C93 & $1.215(6)$ & C71 & $\mathrm{C} 72$ & $1.392(8)$ \\
\hline $\mathrm{O} 30$ & $\mathrm{Li} 2$ & $1.867(8)$ & $\mathrm{C} 73$ & C74 & $1.397(6)$ \\
\hline N1 & $\mathrm{C} 85$ & $1.330(6)$ & $\mathrm{C} 73$ & $\mathrm{C} 78$ & $1.387(7)$ \\
\hline N1 & C89 & $1.336(7)$ & C74 & $\mathrm{C} 75$ & $1.397(7)$ \\
\hline $\mathrm{N} 2$ & C93 & $1.347(7)$ & $\mathrm{C} 75$ & C76 & $1.371(8)$ \\
\hline N2 & C94 & $1.440(7)$ & $\mathrm{C} 76$ & C77 & $1.395(8)$ \\
\hline N2 & C95 & $1.454(8)$ & C77 & C78 & $1.392(7)$ \\
\hline N3 & $\mathrm{C} 102$ & $1.325(7)$ & C79 & C80 & $1.394(10)$ \\
\hline N3 & $\mathrm{C} 103$ & $1.439(8)$ & C79 & C84 & $1.341(10)$ \\
\hline N3 & $\mathrm{C} 104$ & $1.465(8)$ & $\mathrm{C} 80$ & $\mathrm{C} 81$ & $1.367(14)$ \\
\hline N4 & C96 & $1.304(6)$ & $\mathrm{C} 81$ & $\mathrm{C} 82$ & $1.305(18)$ \\
\hline N4 & C97 & $1.452(7)$ & $\mathrm{C} 82$ & $\mathrm{C} 83$ & $1.316(17)$ \\
\hline N4 & C98 & $1.457(7)$ & $\mathrm{C} 83$ & $\mathrm{C} 84$ & $1.413(12)$ \\
\hline N5 & $\mathrm{C} 90$ & $1.315(8)$ & $\mathrm{C} 85$ & $\mathrm{C} 86$ & $1.380(7)$ \\
\hline N5 & C91 & $1.465(9)$ & $\mathrm{C} 86$ & C87 & $1.388(8)$ \\
\hline N5 & C92 & $1.459(9)$ & $\mathrm{C} 87$ & C88 & $1.371(8)$ \\
\hline N6 & C99 & $1.336(12)$ & $\mathrm{C} 88$ & C89 & $1.385(8)$ \\
\hline N6 & C100 & $1.462(18)$ & $\mathrm{O} 31$ & $\mathrm{C} 105$ & $1.254(13)$ \\
\hline N6 & $\mathrm{C} 101$ & $1.456(17)$ & N7 & C105 & $1.323(12)$ \\
\hline N6A & $\mathrm{C} 1 \mathrm{~A}$ & $1.476(16)$ & N7 & $\mathrm{C} 106$ & $1.425(15)$ \\
\hline N6A & $\mathrm{C} 1 \mathrm{~B}$ & $1.460(16)$ & N7 & C107 & $1.479(16)$ \\
\hline N6A & C99 & $1.317(14)$ & C37A & $\mathrm{C} 42 \mathrm{~A}$ & 1.3900 \\
\hline $\mathrm{C} 1$ & $\mathrm{C} 2$ & $1.379(7)$ & $\mathrm{C} 37 \mathrm{~A}$ & $\mathrm{C} 40 \mathrm{~A}$ & 1.3900 \\
\hline $\mathrm{C} 1$ & C6 & $1.418(6)$ & $\mathrm{C} 42 \mathrm{~A}$ & C38A & 1.3900 \\
\hline $\mathrm{C} 2$ & $\mathrm{C} 3$ & $1.395(8)$ & C38A & C39A & 1.3900 \\
\hline $\mathrm{C} 3$ & $\mathrm{C} 4$ & $1.383(9)$ & C39A & $\mathrm{C} 41 \mathrm{~A}$ & 1.3900 \\
\hline $\mathrm{C} 4$ & C5 & $1.357(10)$ & $\mathrm{C} 41 \mathrm{~A}$ & $\mathrm{C} 40 \mathrm{~A}$ & 1.3900 \\
\hline $\mathrm{C} 5$ & C6 & $1.391(8)$ & & & \\
\hline
\end{tabular}

Table S15. Bond Angles for 4.

\begin{tabular}{|c|c|c|c|c|c|c|c|}
\hline Atom & Atom & Atom & Angle ${ }^{\circ}$ & Atom & Atom & Atom & Angle $/^{\circ}$ \\
\hline $\mathrm{O} 1$ & Mn1 & Mn2 & $95.39(8)$ & $\mathrm{C} 85$ & N1 & C89 & $117.0(4)$ \\
\hline $\mathrm{O} 1$ & Mn1 & $\mathrm{O} 2$ & $92.74(12)$ & C89 & N1 & Mn3 & $124.7(3)$ \\
\hline $\mathrm{O} 1$ & Mn1 & O15 & $81.57(11)$ & C93 & $\mathrm{N} 2$ & C94 & $122.4(5)$ \\
\hline $\mathrm{O} 1$ & Mn1 & $\mathrm{O} 27$ & $139.21(12)$ & C93 & $\mathrm{N} 2$ & C95 & $120.6(5)$ \\
\hline $\mathrm{O} 1$ & Mn1 & Li1 & $113.19(19)$ & C94 & $\mathrm{N} 2$ & C95 & $116.9(5)$ \\
\hline $\mathrm{O} 1$ & Mn1 & $\mathrm{Li} 2$ & $40.88(15)$ & $\mathrm{C} 102$ & N3 & C103 & $121.8(6)$ \\
\hline
\end{tabular}




\begin{tabular}{|c|c|c|c|c|c|c|c|}
\hline Atom & Atom & Atom & Angle/ ${ }^{\circ}$ & Atom & Atom & Atom & Angle $/^{\circ}$ \\
\hline $\mathrm{O} 2$ & Mn1 & $\mathrm{Mn} 2$ & $153.24(11)$ & $\mathrm{C} 102$ & N3 & $\mathrm{C} 104$ & $120.1(6)$ \\
\hline $\mathrm{O} 2$ & Mn1 & $\mathrm{O} 15$ & $165.20(13)$ & C103 & N3 & C104 & $118.0(5)$ \\
\hline $\mathrm{O} 2$ & Mn1 & $\mathrm{O} 27$ & $80.69(12)$ & C96 & N4 & C97 & $122.4(5)$ \\
\hline $\mathrm{O} 2$ & Mn1 & Li1 & $39.65(19)$ & C96 & N4 & C98 & $121.2(5)$ \\
\hline $\mathrm{O} 2$ & Mn1 & $\mathrm{Li} 2$ & $132.86(17)$ & C97 & N4 & C98 & $116.4(5)$ \\
\hline $\mathrm{O} 4$ & Mn1 & $\operatorname{Mn} 2$ & 42.61(8) & C90 & N5 & C91 & $119.3(6)$ \\
\hline $\mathrm{O} 4$ & Mn1 & $\mathrm{O} 1$ & $123.87(12)$ & C90 & N5 & C92 & $121.0(6)$ \\
\hline $\mathrm{O} 4$ & Mn1 & $\mathrm{O} 2$ & $113.04(13)$ & C92 & N5 & C91 & 119.1(6) \\
\hline $\mathrm{O} 4$ & Mn1 & $\mathrm{O} 15$ & $81.26(11)$ & C99 & N6 & $\mathrm{C} 100$ & 118.1(19) \\
\hline $\mathrm{O} 4$ & Mn1 & $\mathrm{O} 27$ & $95.19(12)$ & C99 & N6 & C101 & $127(2)$ \\
\hline $\mathrm{O} 4$ & Mn1 & Li1 & $118.3(2)$ & C101 & N6 & $\mathrm{C} 100$ & $115(2)$ \\
\hline $\mathrm{O} 4$ & Mn1 & $\mathrm{Li} 2$ & $94.08(17)$ & $\mathrm{C} 1 \mathrm{~B}$ & N6A & $\mathrm{C} 1 \mathrm{~A}$ & $118(2)$ \\
\hline O15 & Mn1 & $\operatorname{Mn} 2$ & $41.52(7)$ & C99 & N6A & $\mathrm{C} 1 \mathrm{~A}$ & $118.6(17)$ \\
\hline $\mathrm{O} 15$ & Mn1 & $\mathrm{O} 27$ & $94.67(11)$ & C99 & N6A & $\mathrm{C} 1 \mathrm{~B}$ & $123(2)$ \\
\hline $\mathrm{O} 15$ & Mn1 & Li1 & $131.01(19)$ & $\mathrm{C} 2$ & $\mathrm{C} 1$ & Sil & $123.0(4)$ \\
\hline O15 & Mn1 & $\mathrm{Li} 2$ & $45.24(16)$ & $\mathrm{C} 2$ & $\mathrm{C} 1$ & C6 & $117.0(5)$ \\
\hline $\mathrm{O} 27$ & Mn1 & $\operatorname{Mn} 2$ & $108.48(8)$ & C6 & $\mathrm{C} 1$ & Sil & $120.0(4)$ \\
\hline $\mathrm{O} 27$ & Mn1 & Li1 & $42.98(18)$ & $\mathrm{C} 1$ & $\mathrm{C} 2$ & $\mathrm{C} 3$ & $122.1(5)$ \\
\hline $\mathrm{O} 27$ & Mn1 & $\mathrm{Li} 2$ & $136.50(18)$ & $\mathrm{C} 4$ & C3 & $\mathrm{C} 2$ & $120.0(6)$ \\
\hline Li1 & Mn1 & $\operatorname{Mn} 2$ & $149.93(17)$ & $\mathrm{C} 5$ & $\mathrm{C} 4$ & C3 & $119.1(5)$ \\
\hline $\mathrm{Li} 2$ & Mn1 & $\mathrm{Mn} 2$ & $56.19(14)$ & $\mathrm{C} 4$ & C5 & C6 & $121.9(6)$ \\
\hline Li2 & Mn1 & Li1 & $147.3(2)$ & C5 & C6 & $\mathrm{C} 1$ & $120.0(6)$ \\
\hline Mn3 & $\mathrm{Mn} 2$ & Mn1 & $168.88(3)$ & $\mathrm{C} 8$ & C7 & $\mathrm{Si} 2$ & $120.9(4)$ \\
\hline $\mathrm{O} 3$ & $\mathrm{Mn} 2$ & Mn1 & $80.57(8)$ & $\mathrm{C} 8$ & C7 & $\mathrm{C} 12$ & $117.0(5)$ \\
\hline $\mathrm{O} 3$ & $\mathrm{Mn} 2$ & Mn3 & $110.49(8)$ & $\mathrm{C} 12$ & $\mathrm{C} 7$ & $\mathrm{Si} 2$ & $122.0(4)$ \\
\hline $\mathrm{O} 3$ & $\mathrm{Mn} 2$ & $\mathrm{O} 4$ & $94.68(12)$ & C9 & $\mathrm{C} 8$ & $\mathrm{C} 7$ & $121.0(6)$ \\
\hline $\mathrm{O} 3$ & $\mathrm{Mn} 2$ & O14 & $122.65(14)$ & $\mathrm{C} 10$ & C9 & C8 & $120.7(6)$ \\
\hline $\mathrm{O} 3$ & Mn2 & O15 & $88.59(11)$ & C9 & $\mathrm{C} 10$ & $\mathrm{C} 11$ & $120.6(6)$ \\
\hline $\mathrm{O} 3$ & $\mathrm{Mn} 2$ & O16 & $103.94(11)$ & $\mathrm{C} 10$ & $\mathrm{C} 11$ & $\mathrm{C} 12$ & $119.5(6)$ \\
\hline $\mathrm{O} 3$ & $\mathrm{Mn} 2$ & Li2 & $43.80(18)$ & C11 & $\mathrm{C} 12$ & $\mathrm{C} 7$ & $121.3(5)$ \\
\hline $\mathrm{O} 4$ & Mn2 & Mn1 & $40.01(8)$ & $\mathrm{C} 18$ & $\mathrm{C} 13$ & $\mathrm{Si} 3$ & $121.7(6)$ \\
\hline $\mathrm{O} 4$ & $\mathrm{Mn} 2$ & Mn3 & $136.30(8)$ & $\mathrm{C} 18$ & $\mathrm{C} 13$ & $\mathrm{C} 14$ & 120.0 \\
\hline $\mathrm{O} 4$ & Mn2 & O16 & $160.51(12)$ & C14 & C13 & $\mathrm{Si3}$ & $118.2(6)$ \\
\hline $\mathrm{O} 4$ & $\mathrm{Mn} 2$ & $\mathrm{Li} 2$ & $91.59(17)$ & $\mathrm{C} 13$ & $\mathrm{C} 18$ & $\mathrm{C} 17$ & 120.0 \\
\hline O14 & $\mathrm{Mn} 2$ & Mn1 & $133.42(9)$ & $\mathrm{C} 16$ & $\mathrm{C} 17$ & C18 & 120.0 \\
\hline O14 & $\mathrm{Mn} 2$ & Mn3 & $41.73(8)$ & $\mathrm{C} 17$ & $\mathrm{C} 16$ & $\mathrm{C} 15$ & 120.0 \\
\hline $\mathrm{O} 14$ & Mn2 & O4 & $94.65(12)$ & C16 & C15 & C14 & 120.0 \\
\hline $\mathrm{O} 14$ & $\mathrm{Mn} 2$ & $\mathrm{O} 15$ & $148.73(13)$ & C15 & $\mathrm{C} 14$ & $\mathrm{C} 13$ & 120.0 \\
\hline O14 & $\mathrm{Mn} 2$ & O16 & $80.23(11)$ & C14A & C13A & $\mathrm{Si} 3$ & $120.5(12)$ \\
\hline O14 & $\mathrm{Mn} 2$ & $\mathrm{Li} 2$ & $165.69(19)$ & C14A & C13A & C18A & 120.0 \\
\hline $\mathrm{O} 15$ & $\mathrm{Mn} 2$ & Mn1 & 42.92(7) & C18A & C13A & $\mathrm{Si} 3$ & $119.3(12)$ \\
\hline
\end{tabular}




\begin{tabular}{|c|c|c|c|c|c|c|c|}
\hline Atom & Atom & Atom & Angle/ ${ }^{\circ}$ & Atom & Atom & Atom & Angle $/^{\circ}$ \\
\hline $\mathrm{O} 15$ & Mn2 & $\mathrm{Mn} 3$ & $133.40(7)$ & C13A & C14A & $\mathrm{C} 15 \mathrm{~A}$ & 120.0 \\
\hline $\mathrm{O} 15$ & $\mathrm{Mn} 2$ & $\mathrm{O} 4$ & $80.12(11)$ & C16A & $\mathrm{C} 15 \mathrm{~A}$ & C14A & 120.0 \\
\hline $\mathrm{O} 15$ & $\mathrm{Mn} 2$ & O16 & $94.45(10)$ & $\mathrm{C} 15 \mathrm{~A}$ & $\mathrm{C} 16 \mathrm{~A}$ & C17A & 120.0 \\
\hline $\mathrm{O} 15$ & Mn2 & $\mathrm{Li} 2$ & $45.26(17)$ & C16A & C17A & C18A & 120.0 \\
\hline O16 & $\mathrm{Mn} 2$ & Mn1 & $137.35(8)$ & C17A & C18A & C13A & 120.0 \\
\hline O16 & $\mathrm{Mn} 2$ & Mn3 & $40.71(7)$ & $\mathrm{C} 20$ & C19 & $\mathrm{Si} 4$ & $121.4(5)$ \\
\hline O16 & $\mathrm{Mn} 2$ & Li2 & $97.84(17)$ & $\mathrm{C} 20$ & C19 & $\mathrm{C} 24$ & $117.2(6)$ \\
\hline Li2 & $\mathrm{Mn} 2$ & Mn1 & $56.04(15)$ & $\mathrm{C} 24$ & C19 & $\mathrm{Si} 4$ & $121.4(5)$ \\
\hline $\mathrm{Li} 2$ & $\mathrm{Mn} 2$ & Mn3 & $131.34(15)$ & C19 & $\mathrm{C} 20$ & $\mathrm{C} 21$ & $120.7(8)$ \\
\hline O14 & Mn3 & $\mathrm{Mn} 2$ & $39.82(8)$ & $\mathrm{C} 22$ & $\mathrm{C} 21$ & $\mathrm{C} 20$ & $120.8(10)$ \\
\hline $\mathrm{O} 14$ & Mn3 & $\mathrm{O} 26$ & $86.92(15)$ & $\mathrm{C} 23$ & $\mathrm{C} 22$ & $\mathrm{C} 21$ & 119.2(7) \\
\hline O14 & Mn3 & N1 & $164.72(15)$ & $\mathrm{C} 22$ & $\mathrm{C} 23$ & $\mathrm{C} 24$ & $121.5(8)$ \\
\hline O16 & Mn3 & $\mathrm{Mn} 2$ & $42.74(7)$ & C19 & $\mathrm{C} 24$ & $\mathrm{C} 23$ & $120.5(8)$ \\
\hline O16 & Mn3 & $\mathrm{O} 14$ & $80.35(11)$ & $\mathrm{C} 26$ & $\mathrm{C} 25$ & $\mathrm{Si} 5$ & $121.7(5)$ \\
\hline O16 & Mn3 & $\mathrm{O} 26$ & $127.27(14)$ & $\mathrm{C} 30$ & $\mathrm{C} 25$ & $\mathrm{Si} 5$ & $121.1(4)$ \\
\hline O16 & Mn3 & N1 & $96.19(12)$ & $\mathrm{C} 30$ & $\mathrm{C} 25$ & $\mathrm{C} 26$ & $117.3(5)$ \\
\hline $\mathrm{O} 17$ & Mn3 & $\operatorname{Mn} 2$ & $126.76(10)$ & $\mathrm{C} 27$ & $\mathrm{C} 26$ & $\mathrm{C} 25$ & $121.5(8)$ \\
\hline $\mathrm{O} 17$ & Mn3 & $\mathrm{O} 14$ & $102.14(13)$ & $\mathrm{C} 28$ & $\mathrm{C} 27$ & $\mathrm{C} 26$ & $119.9(8)$ \\
\hline $\mathrm{O} 17$ & Mn3 & $\mathrm{O} 16$ & $119.05(13)$ & $\mathrm{C} 29$ & $\mathrm{C} 28$ & $\mathrm{C} 27$ & $120.5(7)$ \\
\hline $\mathrm{O} 17$ & Mn3 & $\mathrm{O} 26$ & $113.63(15)$ & $\mathrm{C} 28$ & C29 & C30 & $120.7(8)$ \\
\hline $\mathrm{O} 17$ & Mn3 & N1 & $92.58(14)$ & $\mathrm{C} 25$ & $\mathrm{C} 30$ & $\mathrm{C} 29$ & $120.1(7)$ \\
\hline $\mathrm{O} 26$ & Mn3 & Mn2 & $101.57(11)$ & C32 & C31 & Si6 & $117.5(4)$ \\
\hline $\mathrm{O} 26$ & Mn3 & N1 & $83.39(16)$ & $\mathrm{C} 32$ & C31 & C36 & 120.0 \\
\hline N1 & Mn3 & $\operatorname{Mn} 2$ & $131.35(10)$ & $\mathrm{C} 36$ & C31 & Si6 & $122.4(4)$ \\
\hline $\mathrm{O} 1$ & Si1 & O9 & $111.00(16)$ & $\mathrm{C} 33$ & $\mathrm{C} 32$ & C31 & 120.0 \\
\hline $\mathrm{O} 1$ & Sil & $\mathrm{O} 12$ & $111.87(17)$ & C34 & C33 & C32 & 120.0 \\
\hline $\mathrm{O} 1$ & Sil & $\mathrm{C} 1$ & 113.9(2) & $\mathrm{C} 33$ & C34 & C35 & 120.0 \\
\hline $\mathrm{O} 1$ & Sil & $\mathrm{Li} 2$ & $26.10(17)$ & $\mathrm{C} 36$ & $\mathrm{C} 35$ & C34 & 120.0 \\
\hline O9 & Sil & $\mathrm{C} 1$ & $105.66(19)$ & $\mathrm{C} 35$ & $\mathrm{C} 36$ & C31 & 120.0 \\
\hline O9 & Sil & Li2 & $91.79(18)$ & C36A & $\mathrm{C} 31 \mathrm{~A}$ & Si6 & $115.7(14)$ \\
\hline $\mathrm{O} 12$ & Sil & O9 & $107.17(18)$ & C36A & C31A & C32A & 120.0 \\
\hline $\mathrm{O} 12$ & Sil & $\mathrm{C} 1$ & $106.8(2)$ & $\mathrm{C} 32 \mathrm{~A}$ & C31A & Si6 & $122.2(14)$ \\
\hline $\mathrm{O} 12$ & Sil & Li2 & $136.74(18)$ & $\mathrm{C} 35 \mathrm{~A}$ & C36A & C31A & 120.0 \\
\hline $\mathrm{C} 1$ & Sil & Li2 & $104.7(2)$ & C34A & $\mathrm{C} 35 \mathrm{~A}$ & C36A & 120.0 \\
\hline O6 & $\mathrm{Si} 2$ & O8 & $110.73(18)$ & C33A & $\mathrm{C} 34 \mathrm{~A}$ & C35A & 120.0 \\
\hline O6 & $\mathrm{Si} 2$ & O9 & $111.73(17)$ & C34A & $\mathrm{C} 33 \mathrm{~A}$ & C32A & 120.0 \\
\hline O6 & $\mathrm{Si} 2$ & C7 & 108.5(2) & C33A & C32A & C31A & 120.0 \\
\hline $\mathrm{O} 8$ & $\mathrm{Si} 2$ & $\mathrm{C} 7$ & $109.1(2)$ & C38 & C37 & $\mathrm{Si} 7$ & $123.3(12)$ \\
\hline O9 & $\mathrm{Si} 2$ & O8 & $108.50(18)$ & $\mathrm{C} 38$ & $\mathrm{C} 37$ & $\mathrm{C} 42$ & 120.0 \\
\hline O9 & $\mathrm{Si} 2$ & $\mathrm{C} 7$ & $108.27(19)$ & $\mathrm{C} 42$ & $\mathrm{C} 37$ & $\mathrm{Si} 7$ & $116.5(13)$ \\
\hline $\mathrm{O} 7$ & $\mathrm{Si} 3$ & O8 & $109.2(2)$ & C37 & C38 & C39 & 120.0 \\
\hline
\end{tabular}




\begin{tabular}{|c|c|c|c|c|c|c|c|}
\hline Atom & Atom & Atom & Angle/ ${ }^{\circ}$ & Atom & Atom & Atom & Angle/ ${ }^{\circ}$ \\
\hline $\mathrm{O} 7$ & $\mathrm{Si} 3$ & C13 & $110.7(4)$ & $\mathrm{C} 40$ & C39 & C38 & 120.0 \\
\hline $\mathrm{O} 7$ & $\mathrm{Si} 3$ & C13A & $109.9(7)$ & $\mathrm{C} 41$ & $\mathrm{C} 40$ & C39 & 120.0 \\
\hline O8 & $\mathrm{Si} 3$ & C13 & $106.9(4)$ & $\mathrm{C} 40$ & C41 & $\mathrm{C} 42$ & 120.0 \\
\hline O8 & $\mathrm{Si} 3$ & C13A & $110.0(8)$ & C41 & $\mathrm{C} 42$ & C37 & 120.0 \\
\hline $\mathrm{O} 10$ & $\mathrm{Si} 3$ & $\mathrm{O} 7$ & $107.4(3)$ & $\mathrm{C} 44$ & $\mathrm{C} 43$ & Si8 & $122.2(4)$ \\
\hline $\mathrm{O} 10$ & $\mathrm{Si} 3$ & O8 & $112.1(2)$ & $\mathrm{C} 48$ & $\mathrm{C} 43$ & $\mathrm{Si} 8$ & $120.8(4)$ \\
\hline $\mathrm{O} 10$ & $\mathrm{Si} 3$ & $\mathrm{C} 13$ & $110.5(3)$ & C48 & $\mathrm{C} 43$ & $\mathrm{C} 44$ & $117.0(5)$ \\
\hline $\mathrm{O} 10$ & $\mathrm{Si} 3$ & C13A & $108.1(7)$ & $\mathrm{C} 45$ & $\mathrm{C} 44$ & $\mathrm{C} 43$ & $121.0(6)$ \\
\hline $\mathrm{O} 2$ & $\mathrm{Si} 4$ & O10 & $112.1(2)$ & $\mathrm{C} 46$ & $\mathrm{C} 45$ & $\mathrm{C} 44$ & $119.2(6)$ \\
\hline $\mathrm{O} 2$ & $\mathrm{Si} 4$ & O12 & $111.86(18)$ & $\mathrm{C} 47$ & $\mathrm{C} 46$ & $\mathrm{C} 45$ & $120.8(5)$ \\
\hline $\mathrm{O} 2$ & $\mathrm{Si} 4$ & C19 & $113.2(2)$ & $\mathrm{C} 46$ & $\mathrm{C} 47$ & $\mathrm{C} 48$ & $119.7(6)$ \\
\hline $\mathrm{O} 2$ & $\mathrm{Si} 4$ & Li1 & $28.7(2)$ & $\mathrm{C} 43$ & $\mathrm{C} 48$ & $\mathrm{C} 47$ & $122.1(6)$ \\
\hline O10 & $\mathrm{Si} 4$ & C19 & $105.2(2)$ & $\mathrm{C} 50$ & C49 & $\mathrm{Si} 9$ & $120(2)$ \\
\hline $\mathrm{O} 10$ & $\mathrm{Si} 4$ & Li1 & $133.3(2)$ & $\mathrm{C} 50$ & C49 & C54 & 120.0 \\
\hline $\mathrm{O} 12$ & $\mathrm{Si} 4$ & O10 & $107.2(2)$ & C54 & C49 & Si9 & $120(2)$ \\
\hline $\mathrm{O} 12$ & $\mathrm{Si} 4$ & C19 & $106.8(2)$ & C49 & $\mathrm{C} 50$ & C51 & 120.0 \\
\hline $\mathrm{O} 12$ & $\mathrm{Si} 4$ & Li1 & $112.1(2)$ & C52 & C51 & $\mathrm{C} 50$ & 120.0 \\
\hline C19 & $\mathrm{Si} 4$ & Li1 & $86.8(2)$ & C51 & C52 & C53 & 120.0 \\
\hline $\mathrm{O} 3$ & $\mathrm{Si} 5$ & O5 & $113.93(18)$ & C54 & C53 & C52 & 120.0 \\
\hline $\mathrm{O} 3$ & $\mathrm{Si} 5$ & O6 & $111.76(17)$ & C53 & C54 & C49 & 120.0 \\
\hline $\mathrm{O} 3$ & $\mathrm{Si} 5$ & $\mathrm{C} 25$ & $111.7(2)$ & C50A & C49A & $\mathrm{Si} 9$ & $119.5(6)$ \\
\hline O5 & Si5 & $\mathrm{C} 25$ & $105.0(2)$ & C50A & C49A & C54A & 120.0 \\
\hline O6 & $\mathrm{Si} 5$ & O5 & $107.09(18)$ & C54A & C49A & Si9 & $120.5(6)$ \\
\hline O6 & $\mathrm{Si} 5$ & $\mathrm{C} 25$ & $106.8(2)$ & C49A & C50A & C51A & 120.0 \\
\hline $\mathrm{O} 5$ & $\mathrm{Si} 6$ & C31 & $112.5(3)$ & C50A & C51A & C52A & 120.0 \\
\hline $\mathrm{O} 5$ & Si6 & C31A & $103.8(10)$ & C51A & C52A & C53A & 120.0 \\
\hline O7 & $\mathrm{Si6}$ & O5 & 109.9(2) & C54A & C53A & C52A & 120.0 \\
\hline $\mathrm{O} 7$ & $\mathrm{Si6}$ & O11 & $109.7(3)$ & C53A & C54A & C49A & 120.0 \\
\hline $\mathrm{O} 7$ & $\mathrm{Si} 6$ & C31 & $106.7(3)$ & C56 & C55 & Si10 & $119.8(3)$ \\
\hline $\mathrm{O} 7$ & $\mathrm{Si6}$ & C31A & $116.7(8)$ & C56 & C55 & C60 & 120.0 \\
\hline O11 & $\mathrm{Si6}$ & O5 & $110.5(2)$ & C60 & C55 & Sil0 & $120.1(3)$ \\
\hline O11 & Si6 & C31 & $107.3(3)$ & $\mathrm{C} 57$ & C56 & C55 & 120.0 \\
\hline O11 & Si6 & C31A & $106.0(10)$ & C56 & C57 & C58 & 120.0 \\
\hline $\mathrm{O} 4$ & $\mathrm{Si} 7$ & O11 & $111.34(19)$ & $\mathrm{C} 57$ & C58 & C59 & 120.0 \\
\hline $\mathrm{O} 4$ & Si7 & O13 & $110.52(18)$ & C60 & C59 & C58 & 120.0 \\
\hline $\mathrm{O} 4$ & Si7 & $\mathrm{C} 37$ & $117.4(9)$ & C59 & C60 & C55 & 120.0 \\
\hline $\mathrm{O} 4$ & $\mathrm{Si} 7$ & $\mathrm{C} 42 \mathrm{~A}$ & $113.6(3)$ & C56A & C55A & Sil0 & 119.7(9) \\
\hline O11 & Si7 & C37 & $112.9(9)$ & C56A & C55A & C60A & 120.0 \\
\hline O11 & Si7 & $\mathrm{C} 42 \mathrm{~A}$ & $106.2(3)$ & $\mathrm{C} 60 \mathrm{~A}$ & C55A & Sil0 & 120.1(9) \\
\hline $\mathrm{O} 13$ & $\mathrm{Si} 7$ & O11 & $106.6(3)$ & C55A & C56A & C57A & 120.0 \\
\hline $\mathrm{O} 13$ & Si7 & C37 & $96.6(8)$ & C58A & C57A & C56A & 120.0 \\
\hline
\end{tabular}




\begin{tabular}{|c|c|c|c|c|c|c|c|}
\hline Atom & Atom & Atom & Angle/ ${ }^{\circ}$ & Atom & Atom & Atom & Angle $/^{\circ}$ \\
\hline $\mathrm{O} 13$ & $\mathrm{Si7}$ & $\mathrm{C} 42 \mathrm{~A}$ & $108.3(3)$ & C57A & C58A & C59A & 120.0 \\
\hline $\mathrm{O} 17$ & $\mathrm{Si} 8$ & O19 & $112.79(18)$ & $\mathrm{C} 60 \mathrm{~A}$ & C59A & C58A & 120.0 \\
\hline $\mathrm{O} 17$ & $\mathrm{Si} 8$ & $\mathrm{O} 20$ & $111.96(18)$ & C59A & C60A & C55A & 120.0 \\
\hline $\mathrm{O} 17$ & $\mathrm{Si} 8$ & C43 & $113.8(2)$ & C62 & C61 & Si11 & $120.2(5)$ \\
\hline O19 & $\mathrm{Si} 8$ & $\mathrm{O} 20$ & $106.57(18)$ & C62 & C61 & C66 & 120.0 \\
\hline O19 & $\mathrm{Si} 8$ & $\mathrm{C} 43$ & $106.2(2)$ & C66 & C61 & Sil1 & $119.8(5)$ \\
\hline $\mathrm{O} 20$ & $\mathrm{Si} 8$ & $\mathrm{C} 43$ & 104.97(19) & C63 & C62 & C61 & 120.0 \\
\hline $\mathrm{O} 18$ & $\mathrm{Si} 9$ & O19 & $112.8(2)$ & C62 & C63 & C64 & 120.0 \\
\hline $\mathrm{O} 18$ & $\mathrm{Si} 9$ & $\mathrm{O} 22$ & 109.32(19) & C65 & C64 & C63 & 120.0 \\
\hline $\mathrm{O} 18$ & $\mathrm{Si} 9$ & C49 & $105.8(12)$ & C64 & C65 & C66 & 120.0 \\
\hline $\mathrm{O} 18$ & $\mathrm{Si} 9$ & C49A & $107.6(4)$ & C65 & C66 & C61 & 120.0 \\
\hline O19 & $\mathrm{Si} 9$ & $\mathrm{O} 22$ & $109.30(18)$ & C62A & C61A & Sil1 & $120.9(12)$ \\
\hline O19 & $\mathrm{Si} 9$ & C49 & $114.0(10)$ & C62A & C61A & C66A & 120.0 \\
\hline O19 & $\mathrm{Si} 9$ & C49A & $107.1(4)$ & C66A & C61A & Si11 & $119.0(12)$ \\
\hline $\mathrm{O} 22$ & $\mathrm{Si} 9$ & C49 & $105.3(11)$ & C63A & C62A & C61A & 120.0 \\
\hline $\mathrm{O} 22$ & $\mathrm{Si} 9$ & C49A & $110.7(4)$ & C62A & C63A & C64A & 120.0 \\
\hline $\mathrm{O} 20$ & Si10 & $\mathrm{O} 21$ & $110.16(17)$ & C65A & C64A & C63A & 120.0 \\
\hline $\mathrm{O} 20$ & Si10 & $\mathrm{O} 23$ & $110.21(17)$ & C64A & C65A & C66A & 120.0 \\
\hline $\mathrm{O} 20$ & Si10 & C55 & $109.0(2)$ & C65A & C66A & C61A & 120.0 \\
\hline $\mathrm{O} 20$ & Si10 & C55A & $102.3(5)$ & C68 & C67 & Si12 & $122.5(4)$ \\
\hline $\mathrm{O} 21$ & Sil0 & C55 & $109.0(2)$ & C72 & C67 & Si12 & $121.3(4)$ \\
\hline $\mathrm{O} 21$ & Sil0 & C55A & $105.7(5)$ & $\mathrm{C} 72$ & C67 & C68 & $116.2(5)$ \\
\hline $\mathrm{O} 23$ & Si10 & $\mathrm{O} 21$ & $110.40(16)$ & C67 & C68 & C69 & $119.6(6)$ \\
\hline $\mathrm{O} 23$ & Si10 & C55 & 108.1(2) & $\mathrm{C} 70$ & C69 & C68 & $120.2(6)$ \\
\hline $\mathrm{O} 23$ & Si10 & C55A & $117.7(6)$ & C71 & $\mathrm{C} 70$ & C69 & $120.5(6)$ \\
\hline $\mathrm{O} 21$ & Sil1 & $\mathrm{O} 22$ & $109.49(17)$ & $\mathrm{C} 70$ & C71 & C72 & $120.9(7)$ \\
\hline $\mathrm{O} 21$ & Sil1 & C61 & $113.0(3)$ & C67 & C72 & C71 & $122.6(6)$ \\
\hline $\mathrm{O} 21$ & Sil1 & C61A & $104.3(8)$ & C74 & C73 & Si13 & $121.6(4)$ \\
\hline $\mathrm{O} 22$ & Sil1 & C61 & $107.9(3)$ & C78 & C73 & Si13 & $120.6(3)$ \\
\hline $\mathrm{O} 22$ & Sil1 & C61A & $113.5(8)$ & C78 & C73 & C74 & $117.8(4)$ \\
\hline $\mathrm{O} 24$ & Si11 & $\mathrm{O} 21$ & $111.21(17)$ & C73 & C74 & C75 & $120.7(5)$ \\
\hline $\mathrm{O} 24$ & Si11 & $\mathrm{O} 22$ & $109.09(17)$ & C76 & $\mathrm{C} 75$ & C74 & $120.4(5)$ \\
\hline $\mathrm{O} 24$ & Sil1 & C61 & 106.0(3) & $\mathrm{C} 75$ & C76 & C77 & $120.0(4)$ \\
\hline $\mathrm{O} 24$ & Sil1 & C61A & $109.2(7)$ & C78 & C77 & C76 & $119.1(5)$ \\
\hline 016 & Si12 & $\mathrm{O} 23$ & $111.12(15)$ & C73 & $\mathrm{C} 78$ & C77 & $121.9(5)$ \\
\hline 016 & Si12 & $\mathrm{O} 25$ & $111.65(15)$ & $\mathrm{C} 80$ & C79 & Sil4 & $120.6(6)$ \\
\hline O16 & Si12 & C67 & $112.8(2)$ & C84 & C79 & Sil4 & $124.3(6)$ \\
\hline $\mathrm{O} 23$ & Si12 & C67 & $108.20(17)$ & C84 & C79 & $\mathrm{C} 80$ & $114.8(7)$ \\
\hline $\mathrm{O} 25$ & Si12 & $\mathrm{O} 23$ & $104.88(16)$ & C81 & $\mathrm{C} 80$ & C79 & $122.8(11)$ \\
\hline $\mathrm{O} 25$ & Si12 & C67 & $107.79(18)$ & $\mathrm{C} 82$ & C81 & $\mathrm{C} 80$ & $120.8(12)$ \\
\hline $\mathrm{O} 15$ & Si13 & $\mathrm{O} 24$ & $108.12(16)$ & C81 & $\mathrm{C} 82$ & $\mathrm{C} 83$ & $119.0(10)$ \\
\hline
\end{tabular}




\begin{tabular}{|c|c|c|c|c|c|c|c|}
\hline Atom & Atom & Atom & Angle $/^{\circ}$ & Atom & Atom & Atom & Angle $/^{\circ}$ \\
\hline $\mathrm{O} 15$ & Si13 & $\mathrm{O} 25$ & $111.28(16)$ & C82 & C83 & C84 & $122.0(11)$ \\
\hline $\mathrm{O} 15$ & Si13 & $\mathrm{C} 73$ & $114.69(17)$ & C79 & C84 & $\mathrm{C} 83$ & $120.4(9)$ \\
\hline $\mathrm{O} 24$ & Si13 & $\mathrm{C} 73$ & 108.13(19) & N1 & $\mathrm{C} 85$ & C86 & $124.3(5)$ \\
\hline $\mathrm{O} 25$ & Si13 & $\mathrm{O} 24$ & $108.01(16)$ & $\mathrm{C} 85$ & C86 & C87 & $117.6(5)$ \\
\hline $\mathrm{O} 25$ & Si13 & $\mathrm{C} 73$ & $106.40(18)$ & C88 & C87 & C86 & $119.4(5)$ \\
\hline $\mathrm{O} 13$ & Si14 & C79 & 107.4(3) & C87 & C88 & C89 & $118.5(5)$ \\
\hline $\mathrm{O} 14$ & Si14 & $\mathrm{O} 13$ & $111.13(17)$ & N1 & C89 & $\mathrm{C} 88$ & $123.2(5)$ \\
\hline $\mathrm{O} 14$ & Sil4 & $\mathrm{O} 18$ & $112.85(19)$ & $\mathrm{O} 26$ & C90 & N5 & $123.6(6)$ \\
\hline $\mathrm{O} 14$ & Sil4 & C79 & $112.6(2)$ & $\mathrm{O} 30$ & C93 & $\mathrm{N} 2$ & $125.2(5)$ \\
\hline O18 & Si14 & $\mathrm{O} 13$ & $105.7(2)$ & $\mathrm{O} 27$ & C96 & N4 & $124.7(4)$ \\
\hline O18 & Sil4 & C79 & $106.7(2)$ & $\mathrm{O} 29$ & C99 & N6 & $126.8(14)$ \\
\hline Sil & $\mathrm{O} 1$ & Mn1 & $133.77(17)$ & $\mathrm{O} 29$ & C99 & N6A & $128.7(13)$ \\
\hline Sil & $\mathrm{O} 1$ & $\mathrm{Li} 2$ & 132.3(3) & $\mathrm{O} 28$ & $\mathrm{C} 102$ & N3 & $123.9(7)$ \\
\hline Li2 & $\mathrm{O} 1$ & Mn1 & $92.8(2)$ & Mn1 & Li1 & $\mathrm{Si} 4$ & $65.15(17)$ \\
\hline $\mathrm{Si} 4$ & $\mathrm{O} 2$ & Mn1 & $127.04(19)$ & $\mathrm{O} 2$ & Li1 & Mn1 & 44.37(19) \\
\hline $\mathrm{Si} 4$ & $\mathrm{O} 2$ & Li1 & $127.9(3)$ & $\mathrm{O} 2$ & Li1 & $\mathrm{Si} 4$ & $23.41(16)$ \\
\hline Li1 & $\mathrm{O} 2$ & Mn1 & $96.0(3)$ & $\mathrm{O} 2$ & Li1 & $\mathrm{O} 27$ & 91.2(4) \\
\hline $\mathrm{Si} 5$ & $\mathrm{O} 3$ & $\operatorname{Mn} 2$ & $135.13(18)$ & $\mathrm{O} 27$ & Li1 & Mn1 & 49.1(2) \\
\hline $\mathrm{Si} 5$ & $\mathrm{O} 3$ & Li2 & $126.5(3)$ & $\mathrm{O} 27$ & Li1 & $\mathrm{Si} 4$ & $113.7(3)$ \\
\hline Li2 & $\mathrm{O} 3$ & $\mathrm{Mn} 2$ & $90.8(2)$ & $\mathrm{O} 28$ & Li1 & Mn1 & $107.1(4)$ \\
\hline Mn1 & $\mathrm{O} 4$ & $\mathrm{Mn} 2$ & $97.38(12)$ & $\mathrm{O} 28$ & Li1 & $\mathrm{Si} 4$ & $100.5(4)$ \\
\hline $\mathrm{Si} 7$ & $\mathrm{O} 4$ & Mn1 & $137.75(17)$ & $\mathrm{O} 28$ & Li1 & $\mathrm{O} 2$ & $114.1(5)$ \\
\hline $\mathrm{Si} 7$ & $\mathrm{O} 4$ & $\operatorname{Mn} 2$ & $124.76(16)$ & $\mathrm{O} 28$ & Li1 & $\mathrm{O} 27$ & $105.1(5)$ \\
\hline $\mathrm{Si6}$ & $\mathrm{O} 5$ & $\mathrm{Si} 5$ & 151.3(2) & $\mathrm{O} 28$ & Li1 & $\mathrm{O} 29$ & $104.7(5)$ \\
\hline $\mathrm{Si} 2$ & O6 & $\mathrm{Si} 5$ & $159.6(3)$ & O29 & Li1 & Mn1 & $147.6(5)$ \\
\hline $\mathrm{Si} 6$ & $\mathrm{O} 7$ & $\mathrm{Si} 3$ & $162.0(4)$ & $\mathrm{O} 29$ & Li1 & $\mathrm{Si} 4$ & $114.9(4)$ \\
\hline $\mathrm{Si3}$ & O8 & $\mathrm{Si} 2$ & $146.9(2)$ & $\mathrm{O} 29$ & Li1 & $\mathrm{O} 2$ & $124.6(5)$ \\
\hline $\mathrm{Si} 2$ & O9 & Sil & $136.2(2)$ & $\mathrm{O} 29$ & Li1 & $\mathrm{O} 27$ & $115.6(5)$ \\
\hline $\mathrm{Si} 3$ & $\mathrm{O} 10$ & $\mathrm{Si} 4$ & $150.0(3)$ & Mn1 & Li2 & $\mathrm{Mn} 2$ & $67.77(15)$ \\
\hline $\mathrm{Si} 6$ & $\mathrm{O} 11$ & $\mathrm{Si} 7$ & $141.4(3)$ & Mn1 & Li2 & Sil & $67.62(16)$ \\
\hline $\mathrm{Si} 4$ & $\mathrm{O} 12$ & Si1 & $129.75(19)$ & $\mathrm{Mn} 2$ & Li2 & Sil & 129.9(3) \\
\hline $\mathrm{Si} 7$ & $\mathrm{O} 13$ & Si14 & $136.0(2)$ & $\mathrm{O} 1$ & Li2 & Mn1 & $46.35(18)$ \\
\hline $\mathrm{Mn} 2$ & $\mathrm{O} 14$ & Mn3 & $98.45(12)$ & $\mathrm{O} 1$ & Li2 & $\mathrm{Mn} 2$ & 111.9(3) \\
\hline Sil4 & $\mathrm{O} 14$ & $\mathrm{Mn} 2$ & 129.04(19) & $\mathrm{O} 1$ & Li2 & Sil & $21.59(14)$ \\
\hline Si14 & $\mathrm{O} 14$ & Mn3 & $127.81(16)$ & O1 & Li2 & $\mathrm{O} 3$ & $112.1(4)$ \\
\hline $\mathrm{Mn} 2$ & $\mathrm{O} 15$ & Mn1 & $95.56(11)$ & $\mathrm{O} 1$ & $\mathrm{Li} 2$ & $\mathrm{O} 15$ & $90.5(3)$ \\
\hline Si13 & $\mathrm{O} 15$ & Mn1 & $132.32(16)$ & $\mathrm{O} 3$ & $\mathrm{Li} 2$ & Mn1 & $90.5(3)$ \\
\hline Si13 & $\mathrm{O} 15$ & $\mathrm{Mn} 2$ & $120.46(14)$ & $\mathrm{O} 3$ & Li2 & $\mathrm{Mn} 2$ & $45.44(16)$ \\
\hline Si13 & $\mathrm{O} 15$ & Li2 & $123.7(3)$ & $\mathrm{O} 3$ & Li2 & Sil & $114.4(3)$ \\
\hline Li2 & $\mathrm{O} 15$ & Mn1 & $85.2(2)$ & $\mathrm{O} 3$ & $\mathrm{Li} 2$ & $\mathrm{O} 15$ & $92.7(3)$ \\
\hline Li2 & $\mathrm{O} 15$ & Mn2 & $86.9(2)$ & $\mathrm{O} 15$ & Li2 & Mn1 & $49.55(17)$ \\
\hline
\end{tabular}




\begin{tabular}{|c|c|c|c|c|c|c|c|}
\hline Atom & Atom & Atom & Angle $/^{\circ}$ & Atom & Atom & Atom & Angle $/^{\circ}$ \\
\hline Mn3 & O16 & $\mathrm{Mn} 2$ & $96.54(11)$ & O15 & Li2 & Mn2 & $47.80(16)$ \\
\hline Si12 & O16 & Mn2 & $128.84(15)$ & $\mathrm{O} 15$ & $\mathrm{Li} 2$ & Sil & $111.5(3)$ \\
\hline Si12 & O16 & Mn3 & $133.82(15)$ & $\mathrm{O} 30$ & $\mathrm{Li} 2$ & Mn1 & $147.2(4)$ \\
\hline $\mathrm{Si} 8$ & O17 & Mn3 & $139.40(19)$ & O30 & $\mathrm{Li} 2$ & $\mathrm{Mn} 2$ & $127.3(3)$ \\
\hline $\mathrm{Si} 9$ & $\mathrm{O} 18$ & Si14 & $151.4(3)$ & O30 & $\mathrm{Li} 2$ & Sil & $102.7(3)$ \\
\hline $\mathrm{Si} 9$ & O19 & $\mathrm{Si} 8$ & $138.5(2)$ & $\mathrm{O} 30$ & $\mathrm{Li} 2$ & $\mathrm{O} 1$ & $118.1(4)$ \\
\hline Si10 & $\mathrm{O} 20$ & Si8 & $144.5(2)$ & O30 & $\mathrm{Li} 2$ & $\mathrm{O} 3$ & $121.1(4)$ \\
\hline Sil1 & $\mathrm{O} 21$ & Sil0 & $156.6(2)$ & O30 & $\mathrm{Li} 2$ & O15 & $114.8(4)$ \\
\hline Sil1 & $\mathrm{O} 22$ & $\mathrm{Si} 9$ & $149.3(2)$ & C105 & N7 & C106 & $120.9(12)$ \\
\hline Sil0 & $\mathrm{O} 23$ & Sil2 & $137.42(19)$ & C105 & N7 & C107 & $122.8(11)$ \\
\hline Si11 & $\mathrm{O} 24$ & Si13 & $146.3(2)$ & C106 & N7 & C107 & $114.9(11)$ \\
\hline Si13 & $\mathrm{O} 25$ & Si12 & 133.01(19) & $\mathrm{O} 31$ & $\mathrm{C} 105$ & N7 & $123.3(13)$ \\
\hline C90 & $\mathrm{O} 26$ & Mn3 & $128.2(4)$ & $\mathrm{C} 42 \mathrm{~A}$ & C37A & $\mathrm{C} 40 \mathrm{~A}$ & 120.0 \\
\hline C96 & $\mathrm{O} 27$ & Mn1 & $122.9(3)$ & C37A & $\mathrm{C} 42 \mathrm{~A}$ & $\mathrm{Si7}$ & $120.2(3)$ \\
\hline C96 & $\mathrm{O} 27$ & Li1 & $132.1(4)$ & C38A & $\mathrm{C} 42 \mathrm{~A}$ & $\mathrm{Si} 7$ & $119.8(3)$ \\
\hline Li1 & $\mathrm{O} 27$ & Mn1 & $87.9(3)$ & C38A & $\mathrm{C} 42 \mathrm{~A}$ & C37A & 120.0 \\
\hline C102 & $\mathrm{O} 28$ & Li1 & $134.3(5)$ & $\mathrm{C} 42 \mathrm{~A}$ & C38A & C39A & 120.0 \\
\hline C99 & O29 & Li1 & $129.2(6)$ & C41A & C39A & C38A & 120.0 \\
\hline C93 & O30 & Li2 & $163.2(5)$ & C39A & C41A & $\mathrm{C} 40 \mathrm{~A}$ & 120.0 \\
\hline C85 & N1 & Mn3 & $118.2(3)$ & C41A & C40A & C37A & 120.0 \\
\hline
\end{tabular}

\section{Experimental}

A single crystal of $\mathrm{C}_{107} \mathrm{H}_{117} \mathrm{Li}_{2} \mathrm{Mn}_{3} \mathrm{~N}_{7} \mathrm{O}_{31} \mathrm{Si}_{14}$ was selected and mounted on a Kappa APEX II DUO diffractometer. The crystal was kept at $100.02 \mathrm{~K}$ during data collection. Using Olex $2^{4}$ as a GUI, the structure was solved with the ShelXS structure solution program using Structure Expansion and refined with the $\mathrm{XL}^{2}$ refinement package using Least Squares minimisation.

\section{Crystal structure determination}

Crystal Data for $\mathrm{C}_{107} \mathrm{H}_{117} \mathrm{Li}_{2} \mathrm{Mn}_{3} \mathrm{~N}_{7} \mathrm{O}_{31} \mathrm{Si}_{14}(M=2569.03 \mathrm{~g} / \mathrm{mol})$ : triclinic, space group P-1 (no. 2), $a=15.499(3) \AA, b=19.247(3) \AA, c=25.649(5) \AA, \alpha=100.410(3)^{\circ}, \beta=92.708(3)^{\circ}, \gamma=$ $97.495(3)^{\circ}, V=7441(2) \AA^{3}, Z=2, T=100.02 \mathrm{~K}, \mu(\mathrm{MoK} \alpha)=0.426 \mathrm{~mm}^{-1}$, Dcalc $=1.147 \mathrm{~g} / \mathrm{cm}^{3}$, 111228 reflections measured $\left(2.944^{\circ} \leq 2 \Theta \leq 56.064^{\circ}\right), 34404$ unique $\left(R_{\text {int }}=0.0409, \mathrm{R}_{\text {sigma }}=\right.$ $0.0514)$ which were used in all calculations. The final $R_{1}$ was $0.0841(\mathrm{I}>2 \sigma(\mathrm{I}))$ and $w R_{2}$ was 0.2268 (all data).

\section{Refinement model description}

Details:

Number of restraints - 1360

1. Fixed Uiso

At 1.2 times of:

All $\mathrm{C}(\mathrm{H})$ groups, All $\mathrm{C}(\mathrm{H}, \mathrm{H})$ groups

At 1.5 times of:

All C(H,H,H) groups 
2. Restrained distances

N6-C99

1.34 with sigma of 0.02

$\mathrm{N} 6 \mathrm{~A}-\mathrm{C} 1 \mathrm{~A}=\mathrm{N} 6-\mathrm{C} 100=\mathrm{N} 6 \mathrm{~A}-\mathrm{C} 1 \mathrm{~B}=\mathrm{N} 6-\mathrm{C} 101$

1.46 with sigma of 0.02

O29-C99

1.25 with sigma of 0.02

N6-C99 = N6A-C99

1.33 with sigma of 0.02

O29-N6

2.26 with sigma of 0.04

O29-N6A

2.26 with sigma of 0.04

C99-C 100

2.43 with sigma of 0.04

C99-C1A

2.43 with sigma of 0.04

C99-C1B

2.43 with sigma of 0.04

C99-C101

2.43 with sigma of 0.04

3. Restrained planarity

O29, N6A, C1A, C1B

with sigma of 0.1

O29, N6, C99, C100, C101

with sigma of 0.1

C99, N6, C100, C101

with sigma of 0.1

C99, N6A, C1A, C1B

with sigma of 0.1

4. Rigid bond restraints

C55, C55A, C56, C56A, C57, C57A, C58, C58A, C59, C59A, C60, C60A

with sigma for 1-2 distances of 0.01 and sigma for 1-3 distances of 0.01

C61, C61A, C62, C62A, C63, C63A, C64, C64A, C65, C65A, C66, C66A

with sigma for 1-2 distances of 0.01 and sigma for 1-3 distances of 0.01

O29, N6, N6A, C1A, C1B, C99, C100, C101

with sigma for 1-2 distances of 0.01 and sigma for 1-3 distances of 0.01

C13, C13A, C14, C14A, C15, C15A, C16, C16A, C17, C17A, C18, C18A with sigma for 1-2 distances of 0.01 and sigma for 1-3 distances of 0.01

C49, C49A, C50, C50A, C51, C51A, C52, C52A, C53, C53A, C54, C54A with sigma for 1-2 distances of 0.01 and sigma for 1-3 distances of 0.01 C31, C32, C33, C34, C35, C36, C31A, C36A, C35A, C34A, C33A, C32A with sigma for 1-2 distances of 0.01 and sigma for 1-3 distances of 0.01 C37, C38, C39, C40, C41, C42, C37A, C42A, C38A, C39A, C41A, C40A with sigma for 1-2 distances of 0.005 and sigma for 1-3 distances of 0.005 5. Uiso/Uaniso restraints and constraints 
$\mathrm{C} 55 \approx \mathrm{C} 55 \mathrm{~A} \approx \mathrm{C} 56 \approx \mathrm{C} 56 \mathrm{~A} \approx \mathrm{C} 57 \approx \mathrm{C} 57 \mathrm{~A} \approx \mathrm{C} 58 \approx \mathrm{C} 58 \mathrm{~A}$

$\approx \mathrm{C} 59 \approx \mathrm{C} 59 \mathrm{~A} \approx \mathrm{C} 60 \approx \mathrm{C} 60 \mathrm{~A}$ : within $2 \mathrm{~A}$ with sigma of 0.04 and

sigma for terminal atoms of 0.08

$\mathrm{C} 61 \approx \mathrm{C} 61 \mathrm{~A} \approx \mathrm{C} 62 \approx \mathrm{C} 62 \mathrm{~A} \approx \mathrm{C} 63 \approx \mathrm{C} 63 \mathrm{~A} \approx \mathrm{C} 64 \approx \mathrm{C} 64 \mathrm{~A}$

$\approx \mathrm{C} 65 \approx \mathrm{C} 65 \mathrm{~A} \approx \mathrm{C} 66 \approx \mathrm{C} 66 \mathrm{~A}$ : within $2 \mathrm{~A}$ with sigma of 0.04 and

sigma for terminal atoms of 0.08

$\mathrm{O} 29 \approx \mathrm{N} 6 \approx \mathrm{N} 6 \mathrm{~A} \approx \mathrm{C} 1 \mathrm{~A} \approx \mathrm{C} 1 \mathrm{~B} \approx \mathrm{C} 99 \approx \mathrm{C} 100 \approx \mathrm{C} 101$ :

within $2 \mathrm{~A}$ with sigma of 0.04 and sigma for terminal atoms of 0.08

$\mathrm{C} 13 \approx \mathrm{C} 13 \mathrm{~A} \approx \mathrm{C} 14 \approx \mathrm{C} 14 \mathrm{~A} \approx \mathrm{C} 15 \approx \mathrm{C} 15 \mathrm{~A} \approx \mathrm{C} 16 \approx \mathrm{C} 16 \mathrm{~A}$

$\approx \mathrm{C} 17 \approx \mathrm{C} 17 \mathrm{~A} \approx \mathrm{C} 18 \approx \mathrm{C} 18 \mathrm{~A}$ : within $2 \mathrm{~A}$ with sigma of 0.04 and

sigma for terminal atoms of 0.08

$\mathrm{C} 49 \approx \mathrm{C} 49 \mathrm{~A} \approx \mathrm{C} 50 \approx \mathrm{C} 50 \mathrm{~A} \approx \mathrm{C} 51 \approx \mathrm{C} 51 \mathrm{~A} \approx \mathrm{C} 52 \approx \mathrm{C} 52 \mathrm{~A}$

$\approx \mathrm{C} 53 \approx \mathrm{C} 53 \mathrm{~A} \approx \mathrm{C} 54 \approx \mathrm{C} 54 \mathrm{~A}$ : within $2 \mathrm{~A}$ with sigma of 0.04 and

sigma for terminal atoms of 0.08

$\mathrm{C} 31 \approx \mathrm{C} 32 \approx \mathrm{C} 33 \approx \mathrm{C} 34 \approx \mathrm{C} 35 \approx \mathrm{C} 36 \approx \mathrm{C} 31 \mathrm{~A} \approx \mathrm{C} 36 \mathrm{~A}$

$\approx \mathrm{C} 35 \mathrm{~A} \approx \mathrm{C} 34 \mathrm{~A} \approx \mathrm{C} 33 \mathrm{~A} \approx \mathrm{C} 32 \mathrm{~A}$ : within $2 \mathrm{~A}$ with sigma of 0.04 and

sigma for terminal atoms of 0.08

$\mathrm{C} 37 \approx \mathrm{C} 38 \approx \mathrm{C} 39 \approx \mathrm{C} 40 \approx \mathrm{C} 41 \approx \mathrm{C} 42 \approx \mathrm{C} 37 \mathrm{~A} \approx \mathrm{C} 42 \mathrm{~A}$

$\approx \mathrm{C} 38 \mathrm{~A} \approx \mathrm{C} 39 \mathrm{~A} \approx \mathrm{C} 41 \mathrm{~A} \approx \mathrm{C} 40 \mathrm{~A}$ : within $2 \mathrm{~A}$ with sigma of 0.005 and

sigma for terminal atoms of 0.01

6. Others

$\operatorname{Sof}(\mathrm{C} 55 \mathrm{~A})=\operatorname{Sof}(\mathrm{C} 56 \mathrm{~A})=\operatorname{Sof}(\mathrm{H} 56 \mathrm{~A})=\operatorname{Sof}(\mathrm{C} 57 \mathrm{~A})=\operatorname{Sof}(\mathrm{H} 57 \mathrm{~A})=\operatorname{Sof}(\mathrm{C} 58 \mathrm{~A})=\operatorname{Sof}(\mathrm{H} 58 \mathrm{~A})=$

$\operatorname{Sof}(\mathrm{C} 59 \mathrm{~A})=\operatorname{Sof}(\mathrm{H} 59 \mathrm{~A})=\operatorname{Sof}(\mathrm{C} 60 \mathrm{~A})=\operatorname{Sof}(\mathrm{H} 60 \mathrm{~A})=1-\mathrm{FVAR}(1)$

$\operatorname{Sof}(\mathrm{C} 55)=\operatorname{Sof}(\mathrm{C} 56)=\operatorname{Sof}(\mathrm{H} 56)=\operatorname{Sof}(\mathrm{C} 57)=\operatorname{Sof}(\mathrm{H} 57)=\operatorname{Sof}(\mathrm{C} 58)=\operatorname{Sof}(\mathrm{H} 58)=\operatorname{Sof}(\mathrm{C} 59)=$

$\operatorname{Sof}(\mathrm{H} 59)=\operatorname{Sof}(\mathrm{C} 60)=\operatorname{Sof}(\mathrm{H} 60)=\mathrm{FVAR}(1)$

$\operatorname{Sof}(\mathrm{C61A})=\operatorname{Sof}(\mathrm{C} 62 \mathrm{~A})=\operatorname{Sof}(\mathrm{H} 62 \mathrm{~A})=\operatorname{Sof}(\mathrm{C63A})=\operatorname{Sof}(\mathrm{H} 63 \mathrm{~A})=\operatorname{Sof}(\mathrm{C64A})=\operatorname{Sof}(\mathrm{H} 64 \mathrm{~A})=$

$\operatorname{Sof}(\mathrm{C} 65 \mathrm{~A})=\operatorname{Sof}(\mathrm{H} 65 \mathrm{~A})=\operatorname{Sof}(\mathrm{C} 66 \mathrm{~A})=\operatorname{Sof}(\mathrm{H} 66 \mathrm{~A})=1-\mathrm{FVAR}(2)$

$\operatorname{Sof}(\mathrm{C} 61)=\operatorname{Sof}(\mathrm{C} 62)=\operatorname{Sof}($ H62 $)=\operatorname{Sof}(\mathrm{C} 63)=\operatorname{Sof}($ H63 $)=\operatorname{Sof}(\mathrm{C} 64)=\operatorname{Sof}($ H64 $)=\operatorname{Sof}(\mathrm{C} 65)=$

$\operatorname{Sof}(\mathrm{H} 65)=\operatorname{Sof}(\mathrm{C} 66)=\operatorname{Sof}(\mathrm{H} 66)=\mathrm{FVAR}(2)$

$\operatorname{Sof}(\mathrm{N} 6 \mathrm{~A})=\operatorname{Sof}(\mathrm{C} 1 \mathrm{~A})=\operatorname{Sof}(\mathrm{H} 1 \mathrm{AA})=\operatorname{Sof}(\mathrm{H} 1 \mathrm{AB})=\operatorname{Sof}(\mathrm{H} 1 \mathrm{AC})=\operatorname{Sof}(\mathrm{C} 1 \mathrm{~B})=\operatorname{Sof}(\mathrm{H} 1 \mathrm{BA})=\operatorname{Sof}(\mathrm{H} 1 \mathrm{BB}$

)$=$

$\operatorname{Sof}(\mathrm{H} 1 \mathrm{BC})=\operatorname{Sof}(\mathrm{H} 99 \mathrm{~A})=1-\mathrm{FVAR}(3)$

$\operatorname{Sof}(\mathrm{N} 6)=\operatorname{Sof}(\mathrm{H} 99)=\operatorname{Sof}(\mathrm{C} 100)=\operatorname{Sof}(\mathrm{H} 10 \mathrm{~A})=\operatorname{Sof}(\mathrm{H} 10 \mathrm{~B})=\operatorname{Sof}(\mathrm{H} 10 \mathrm{C})=\operatorname{Sof}(\mathrm{C} 101)=\operatorname{Sof}(\mathrm{H} 10 \mathrm{D})=$

Sof $(\mathrm{H} 10 \mathrm{E})=\operatorname{Sof}(\mathrm{H} 10 \mathrm{~F})=$ FVAR $(3)$

$\operatorname{Sof}(\mathrm{C} 13 \mathrm{~A})=\operatorname{Sof}(\mathrm{C} 14 \mathrm{~A})=\operatorname{Sof}(\mathrm{H} 14 \mathrm{~A})=\operatorname{Sof}(\mathrm{C} 15 \mathrm{~A})=\operatorname{Sof}(\mathrm{H} 15 \mathrm{~A})=\operatorname{Sof}(\mathrm{C} 16 \mathrm{~A})=\operatorname{Sof}(\mathrm{H} 16 \mathrm{~A})=$

$\operatorname{Sof}(\mathrm{C} 17 \mathrm{~A})=\operatorname{Sof}(\mathrm{H} 17 \mathrm{~A})=\operatorname{Sof}(\mathrm{C} 18 \mathrm{~A})=\operatorname{Sof}(\mathrm{H} 18 \mathrm{~A})=1-\mathrm{FVAR}(4)$

$\operatorname{Sof}(\mathrm{C} 13)=\operatorname{Sof}(\mathrm{C} 18)=\operatorname{Sof}(\mathrm{H} 18)=\operatorname{Sof}(\mathrm{C} 17)=\operatorname{Sof}(\mathrm{H} 17)=\operatorname{Sof}(\mathrm{C} 16)=\operatorname{Sof}(\mathrm{H} 16)=\operatorname{Sof}(\mathrm{C} 15)=$

$\operatorname{Sof}(\mathrm{H} 15)=\operatorname{Sof}(\mathrm{C} 14)=\operatorname{Sof}(\mathrm{H} 14)=$ FVAR $(4)$

$\operatorname{Sof}(\mathrm{C} 49 \mathrm{~A})=\operatorname{Sof}(\mathrm{C} 50 \mathrm{~A})=\operatorname{Sof}(\mathrm{H} 50 \mathrm{~A})=\operatorname{Sof}(\mathrm{C} 51 \mathrm{~A})=\operatorname{Sof}(\mathrm{H} 51 \mathrm{~A})=\operatorname{Sof}(\mathrm{C} 52 \mathrm{~A})=\operatorname{Sof}(\mathrm{H} 52 \mathrm{~A})=$

$\operatorname{Sof}(\mathrm{C} 53 \mathrm{~A})=\operatorname{Sof}(\mathrm{H} 53 \mathrm{~A})=\operatorname{Sof}(\mathrm{C} 54 \mathrm{~A})=\operatorname{Sof}(\mathrm{H} 54 \mathrm{~A})=1-\mathrm{FVAR}(5)$

$\operatorname{Sof}(\mathrm{C} 49)=\operatorname{Sof}(\mathrm{C} 50)=\operatorname{Sof}(\mathrm{H} 50)=\operatorname{Sof}(\mathrm{C} 51)=\operatorname{Sof}(\mathrm{H} 51)=\operatorname{Sof}(\mathrm{C} 52)=\operatorname{Sof}(\mathrm{H} 52)=\operatorname{Sof}(\mathrm{C} 53)=$

$\operatorname{Sof}(\mathrm{H} 53)=\operatorname{Sof}(\mathrm{C} 54)=\operatorname{Sof}(\mathrm{H} 54)=$ FVAR(5)

$\operatorname{Sof}(\mathrm{C} 31 \mathrm{~A})=\operatorname{Sof}(\mathrm{C} 36 \mathrm{~A})=\operatorname{Sof}(\mathrm{H} 36 \mathrm{~A})=\operatorname{Sof}(\mathrm{C} 35 \mathrm{~A})=\operatorname{Sof}(\mathrm{H} 35 \mathrm{~A})=\operatorname{Sof}(\mathrm{C} 34 \mathrm{~A})=\operatorname{Sof}(\mathrm{H} 34 \mathrm{~A})=$

$\operatorname{Sof}(\mathrm{C} 33 \mathrm{~A})=\operatorname{Sof}(\mathrm{H} 33 \mathrm{~A})=\operatorname{Sof}(\mathrm{C} 32 \mathrm{~A})=\operatorname{Sof}(\mathrm{H} 32 \mathrm{~A})=1-\mathrm{FVAR}(6)$

$\operatorname{Sof}(\mathrm{C} 31)=\operatorname{Sof}(\mathrm{C} 32)=\operatorname{Sof}(\mathrm{H} 32)=\operatorname{Sof}(\mathrm{C} 33)=\operatorname{Sof}(\mathrm{H} 33)=\operatorname{Sof}(\mathrm{C} 34)=\operatorname{Sof}(\mathrm{H} 34)=\operatorname{Sof}(\mathrm{C} 35)=$ 
$\operatorname{Sof}(\mathrm{H} 35)=\operatorname{Sof}(\mathrm{C} 36)=\operatorname{Sof}(\mathrm{H} 36)=$ FVAR $(6)$

$\operatorname{Sof}(C 37 A)=\operatorname{Sof}(H 37 A)=\operatorname{Sof}(C 42 A)=\operatorname{Sof}(C 38 A)=\operatorname{Sof}(H 38 A)=\operatorname{Sof}(C 39 A)=\operatorname{Sof}(H 39 A)=$

$\operatorname{Sof}(\mathrm{C} 41 \mathrm{~A})=\operatorname{Sof}(\mathrm{H} 41 \mathrm{~A})=\operatorname{Sof}(\mathrm{C} 40 \mathrm{~A})=\operatorname{Sof}(\mathrm{H} 40 \mathrm{~A})=1-\mathrm{FVAR}(7)$

$\operatorname{Sof}(\mathrm{C} 37)=\operatorname{Sof}(\mathrm{C} 38)=\operatorname{Sof}(\mathrm{H} 38)=\operatorname{Sof}(\mathrm{C} 39)=\operatorname{Sof}(\mathrm{H} 39)=\operatorname{Sof}(\mathrm{C} 40)=\operatorname{Sof}(\mathrm{H} 40)=\operatorname{Sof}(\mathrm{C} 41)=$

$\operatorname{Sof}(\mathrm{H} 41)=\operatorname{Sof}(\mathrm{C} 42)=\operatorname{Sof}(\mathrm{H} 42)=\operatorname{FVAR}(7)$

7.a Aromatic/amide $\mathrm{H}$ refined with riding coordinates:

C2(H2), C3(H3), C4(H4), C5(H5), C6(H6), C8(H8), C9(H9), C10(H10), C11(H11),

C12(H12), C18(H18), C17(H17), C16(H16), C15(H15), C14(H14), C14A(H14A),

C15A(H15A), C16A(H16A), C17A(H17A), C18A(H18A), C20(H20), C21(H21), C22(H22),

C23(H23), C24(H24), C26(H26), C27(H27), C28(H28), C29(H29), C30(H30), C32(H32),

C33(H33), C34(H34), C35(H35), C36(H36), C36A(H36A), C35A(H35A), C34A(H34A),

C33A(H33A), C32A(H32A), C38(H38), C39(H39), C40(H40), C41(H41), C42(H42),

C44(H44), C45(H45), C46(H46), C47(H47), C48(H48), C50(H50), C51(H51), C52(H52),

C53(H53), C54(H54), C50A(H50A), C51A(H51A), C52A(H52A), C53A(H53A),

C54A(H54A), C56(H56), C57(H57), C58(H58), C59(H59), C60(H60), C56A(H56A),

C57A(H57A), C58A(H58A), C59A(H59A), C60A(H60A), C62(H62), C63(H63), C64(H64),

C65(H65), C66(H66), C62A(H62A), C63A(H63A), C64A(H64A), C65A(H65A),

C66A(H66A),

C68(H68), C69(H69), C70(H70), C71(H71), C72(H72), C74(H74), C75(H75),

C76(H76), C77(H77), C78(H78), C80(H80), C81(H81), C82(H82), C83(H83), C84(H84),

C85(H85), C86(H86), C87(H87), C88(H88), C89(H89), C90(H90), C93(H93),

C96(H96), C99(H99), C99(H99A), C102(H102), C105(H105), C37A(H37A), C38A(H38A),

C39A(H39A), C41A(H41A), C40A(H40A)

7.b Fitted hexagon refined as free rotating group:

C13(C18,C17,C16,C15,C14), C13A(C14A,C15A,C16A,C17A,C18A), C31(C32,C33,C34,C35, C36), C31A(C36A,C35A,C34A,C33A,C32A), C37(C38,C39,C40,C41,C42), C49(C50,C51, C52,C53,C54), C49A(C50A,C51A,C52A,C53A,C54A), C55(C56,C57,C58,C59,C60), C55A(C56A,C57A,C58A,C59A,C60A), C61(C62,C63,C64,C65,C66),

C61A(C62A,C63A,C64A,

C65A,C66A), C37A(C42A,C38A,C39A,C41A,C40A)

7.c Idealised $\mathrm{Me}$ refined as rotating group:

C1A(H1AA,H1AB,H1AC), C1B(H1BA,H1BB,H1BC), C91(H91A,H91B,H91C), C92(H92A,H92B,

H92C), C94(H94A,H94B,H94C), C95(H95A,H95B,H95C), C97(H97A,H97B,H97C),

C98(H98A,

H98B,H98C), C100(H10A,H10B,H10C), C101(H10D,H10E,H10F), C103(H10G,H10H,H10I), C104(H10J,H10K,H10L), C106(H10M,H10N,H10O), C107(H10P,H10Q,H10R)

This report has been created with Olex2, compiled on 2018.05.29 svn.r3508 for OlexSys. 


\section{References}

(1) Andersen, R. A.; Faegri, K.; Green, J. C.; Haaland, A.; Lappert, M. F.; Leung, W. P.; Rypdal, K. Synthesis of bis[bis(trimethylsilyl)amido]iron(II). Structure and bonding in $\mathrm{M}[\mathrm{N}(\mathrm{SiMe} 3) 2] 2(\mathrm{M}=$ manganese, iron, cobalt): two-coordinate transition-metal amides. Inorganic Chemistry 1988, 27, 1782-1786.

(2) Sheldrick, G. M. A short history of SHELX. Acta Crystallographica 2008, A64, 112-122.

(3) LeSuer, R. J.; Buttolph, C.; Geiger, W. E. Comparison of the Conductivity Properties of the Tetrabutylammonium Salt of Tetrakis(pentafluorophenyl)borate Anion with Those of Traditional Supporting Electrolyte Anions in Nonaqueous Solvents. Analytical Chemistry 2004, 76, 63956401.

(4) Dolomanov, O. V.; Bourhis, L. J.; Gildea, R. J.; Howard, J. A. K.; Puschmann, H. OLEX2: a complete structure solution, refinement and analysis program. Journal of Applied Crystallography 2009, 42, 339-341. 